\title{
Widespread multi-targeted therapy resistance via drug-induced secretome fucosylation
}

Mark Borris D. Aldonza ${ }^{1,2,3,4,5, \dagger}$, Junghwa Cha ${ }^{3,6, \dagger}$, Insung Yong ${ }^{6, \#}$, Jayoung Ku ${ }^{1,3, \#}$, Dabin Lee ${ }^{4,5}$, Pavel Sinitcyn ${ }^{7}$, Ryeong-Eun Cho ${ }^{1,3}$, Roben D. Delos Reyes ${ }^{8}$, Dongwook Kim ${ }^{4,5}$, Hye-Jin Sung ${ }^{4,5}$, Soyeon Kim ${ }^{9,10}$, Minjeong Kang ${ }^{1,3}$, Yongsuk Ku ${ }^{1,3}$, Geonho Park ${ }^{1}$, Han Suk Ryu ${ }^{11}$, Sukki Cho ${ }^{12}$, Tae Min Kim ${ }^{9,10}$, Pilnam Kim ${ }^{3,6, \ddagger, *}$, Je-Yoel Cho ${ }^{4,5, \neq, *}$, Yoosik Kim ${ }^{1,3, \ddagger, *}$

${ }^{1}$ Department of Chemical and Biomolecular Engineering, Korea Advanced Institute of Sciences and Technology (KAIST), Daejeon 34141, Korea.

${ }^{2}$ Department of Biological Sciences, KAIST, Daejeon 34141, Korea.

${ }^{3} \mathrm{KI}$ for Health Science and Technology (KIHST), KAIST, Daejeon 34141, Korea.

${ }^{4}$ Department of Biochemistry, College of Veterinary Medicine, Seoul National University, Seoul 151-742, Korea.

${ }^{5}$ BK21 PLUS Program for Creative Veterinary Science Research and Research Institute for Veterinary Science, Seoul National University, Seoul 151-742, Korea.

${ }^{6}$ Department of Bio and Brain Engineering, KAIST, Daejeon 34141, Korea.

${ }^{7}$ Computational Systems Biochemistry Research Group, Max Planck Institute of Biochemistry, 82152 Martinsried, Germany.

${ }^{8}$ Department of Electrical Engineering, KAIST, Daejeon 34141, Korea.

${ }^{9}$ Department of Internal Medicine, Seoul National University Hospital, Seoul 03080, Korea.

${ }^{10}$ Cancer Research Institute, Seoul National University College of Medicine, Seoul 03080, Korea.

${ }^{11}$ Department of Pathology, Seoul National University Hospital, Seoul National University College of Medicine, Seoul 03080, Korea.

${ }^{12}$ Department of Thoracic and Cardiovascular Surgery, Seoul National University Bundang Hospital, Seongnam 13620, Republic of Korea.

*Corresponding author. Email: ysyoosik@kaist.ac.kr (Y.K.); jeycho@snu.ac.kr (J.Y.C.); pkim@kaist.ac.kr (P.K.)

†These authors contributed equally to this work as co-first authors.

\#These authors contributed equally to this work as co-second authors.

¥These authors contributed equally to this work as co-senior authors.

Running title: Targeted therapy resistance via secretome fucosylation. 
Abstract

Cancer secretome is a reservoir for aberrant glycosylation. How therapies alter this posttranslational cancer hallmark and the consequences thereof remain elusive. Here we show that an elevated secretome fucosylation is a pan-cancer signature of both response and resistance to multiple targeted therapies. Large-scale pharmacogenomics revealed that fucosylation genes display widespread association with resistance to these therapies. In both cancer cell cultures and patients, targeted kinase inhibitors distinctively induced core fucosylation of secreted proteins less than $60 \mathrm{kDa}$. Label-free proteomics of $\mathrm{N}$ glycoproteomes revealed that fucosylation of the antioxidant PON1 is a critical component of the therapy-induced secretome. Core fucosylation in the Golgi impacts PON1 stability and folding prior to secretion, promoting a more degradation-resistant PON1. Non-specific and PON1-specific secretome de-N-glycosylation both limited the expansion of resistant clones in a tumor regression model. Our findings demonstrate that core fucosylation is a common modification indirectly induced by targeted therapies that paradoxically promotes resistance.

Keywords: fucosylation, n-linked glycosylation, targeted therapy, secretome, resistance 


\section{Introduction}

Complete responses to targeted therapies remain rare for a vast majority of cancer patients ${ }^{[1]}$. While long-term disease stabilization can be achieved by therapeutic inhibition of oncogenic drivers, resistance to this targeted strategy is inevitable ${ }^{[1,2,3]}$. In the clinic, partial remission can be achieved by classes of inhibitors that target amplified or mutationally activated kinases such as EGFR mutations or ALK translocations in lung adenocarcinoma, BRAF mutations in melanoma, or HER2 amplifications in breast cancer ${ }^{[3,4,5]}$. Both genetic and non-genetic mechanisms of resistance to these inhibitors exist ${ }^{[6]}$. However, the innate nature of many of these resistance acquisition models precludes the critical role of the tumor microenvironment (TME) in contributing to an incomplete tumor regression after therapy. For instance, a complex network of secreted signals from drug-stressed tumors termed therapy-induced secretomes (TIS) was shown to facilitate the selective expansion of a small number of pre-existing resistant clones, paradoxically explaining relapse to targeted therapy ${ }^{[7]}$. Systemic understanding of this therapy-induced niche could lead to a paradigm shift in our current management of clinical drug resistance in cancer.

The cancer secretome comprises a set of secreted proteins that is pro-tumorigenic in nature. Many components of this secretome serve as disease biomarkers and are major druggable targets ${ }^{[8]}$. Both classical and non-classical pathways regulate the secretion of these components including extracellular matrix proteins, exosomes, growth factors, cytokines, shed receptors, and proteases ${ }^{[8,9,10]}$. During stress, these secretome components are remodeled depending on tissue architecture and cell composition of the TME, stress-inducing stimuli, or conditions that affect liver homeostasis-a systemic dictator of the secretome and plasma proteome states ${ }^{[11,12]}$. Substantially, secreted soluble proteins undergo post-translational modifications (PTMs) that functionally predominate their trafficking, stability, and folding prior to secretion ${ }^{[13]}$. These PTMs in the secretory pathway are constantly employed to form tumorigenic niches upon chemotherapy, radiotherapy, targeted therapy, or immunotherapy ${ }^{[13,14,15]}$. Among these PTMs, phosphorylation and glycosylation are the most common. Glycosylation-the covalent addition of sugar moieties to target scaffolds-is the most abundant PTM of the secretome, as nearly all secreted mammalian proteins have at least one glycan, a sugar-based assembly, attached to them at a specific site $^{[16,17]}$. For example, therapy-induced apoptotic disassembly of the Golgi is associated with the anomalous synthesis of specific glycan types ${ }^{[18,19]}$. In some cases, direct glycosylation of apoptotic signals upon therapy can restrain or trigger their cell killing capacity ${ }^{[20]}$. Moreover, therapies that act as endoplasmic reticulum (ER) stressors can inhibit protein glycosylation and reduce disulfide bonds initiating an unfolded protein response (UPR) ${ }^{[21,22]}$. While there is little evidence suggesting a post-ER quality control that operates at the Golgi following UPR, stress-induced regulation of terminal glycosylation is a complementary mechanism of Golgilocalized machinery that predominates the assembly of newly synthesized secretory proteins $^{[23]}$.

An abnormal glycome is a cancer hallmark ${ }^{[24]}$. Cancer-specific changes in two of the most frequent glycosylation types, $\mathrm{O}$ - and $\mathrm{N}$-linked glycosylation, are coordinated with expression of genes encoding for glycosyltransferases-enzymes that catalyze glycosidic linkages-and glycosidases-enzymes that cleave glycosidic bonds-and their localization within the secretory pathway (Golgi apparatus and ER) ${ }^{[24]}$. Although we note that expression of other enzymecoding genes (i.e., those involved in sugar metabolism and transport and glycan sulfation) are also relevant for understanding aberrant glycosylation. Malignant transformation predominantly displays distinct $\mathrm{N}$-glycomes. Throughout this process, unique alterations in both glycan level and composition, their conjugation and linkages, are reflected in the cell surface, intracellular, and extracellular scaffolds of mostly lipids and proteins ${ }^{[25,26]}$. Lewis antigens, components of exocrine epithelial secretions, are among the most frequently 
overexpressed fucosylated epitopes during carcinogenesis ${ }^{[27]}$. Most obviously, this is attributed to the extensive activity of glycosyltransferases, mainly by fucosyltransferases $(\text { FUTs })^{[28]}$. However, more nuanced and complicated dysregulations can arise from incomplete synthesis-truncated glycosylation common in early carcinogenesis-or neo-synthesis-de novo production of atypical glycosylation patterns-which are mediated by a complex interplay of glycosyltransferases such as FUTs and other factors that regulate fucose metabolism in the Golgi/ER ${ }^{[17,29]}$. As a result, several types of Lewis antigens, including sialylated Lewis structures, are currently being utilized in the clinic as prognostic cancer biomarkers ${ }^{[27,29]}$. Given that these glycan alterations influence the cancer secretome, therapy-induced remodeling of the local TME, particularly its secreted components, must involve modified functionalities in the multi-step process of glycosylation.

Here, we identify that core fucosylation, modification at the $\mathrm{N}$-glycan core, is a major posttranslational signature of the pan-cancer TIS. Using pharmacogenomics, label-free proteomics, and a panoply of perturbation assays, we reveal that the therapy-induced aberration in secretome fucosylation involves (i) a differential induction of relatively smaller fucosylated proteins ( $<60 \mathrm{kDa}$ ), (ii) $\alpha 1,6$-fucosyltransferase (FUT8)-dependent transfer of GDP- $\beta$-I-fucose (GDP-Fuc) onto N-glycan core structures in the Golgi compartment, (iii) expression of fucose salvage genes and the GDP-Fuc transporter SLC35C1, and most significantly, (iv) core fucosylation of the antioxidant paraoxonase 1 (PON1). By utilizing several cellular models of drug resistance paired with patient specimens, we show that an elevated secretome fucosylation is likely a complementary mechanism of cancer relapse and targeted therapy resistance. In addition to uncovering the regulation of this TIS modification, we tested the functional consequences of generally blocking secretome core fucosylation or specifically constraining fucosylated PON1. Indeed, secretome de-N-glycosylation by a glycosidase, fucosylation inhibition by FUT8 or SLC35C1 RNA interference (RNAi), or sitespecific blockade of PON1 core fucosylation dramatically prevented TIS-directed rebound of minority resistant clone population in a regressing heterogeneous cell pool. Furthermore, a targeted screen and transcriptome-wide gene expression analysis unveil effectors of redox stress sensing and the UPR as secretome fucosylation-specific resistance modulators. Our findings point to a new view of the TIS that extends its role in establishing a resistancepromoting microenvironment niche via core fucosylation.

\section{Results}

\section{Core fucosylation of therapy-induced cancer secretomes.}

While fucose is naturally present in a variety of glycolipids and glycoproteins, fucose moieties on N-glycans of secreted proteins are often dysregulated in cancer and are among the most aberrant sugar moieties of cancer glycoproteomes ${ }^{[16]}$. How therapies alter their on-site linkages and regulate their overall levels remain obscure. We investigated whether fucosylation is correlated with drug sensitivity by comprehensive mining of available data on genes involved in fucose metabolism (FUK, FPGT, FX, GMDS), fucosylation branching [FUTs, protein O-fucosyltransferases (POFUTs)], and GDP-Fuc transport (SLC35C1) in the Genomics of Drug Sensitivity in Cancer (GDSC) and the Cancer Cell Line Encyclopedia (CCLE), two of the largest publicly available pharmacogenomics data sets ${ }^{[30,31]}$. We first evaluated the consistency of the pharmacogenomic data from the two datasets. Comparative analysis using the correlation between FUT gene expression and overall drug sensitivity (IC50 for GDSC and area under the curve, AUC for CCLE) as a metric showed that the molecular data are in concordance despite the apparent differences in cell lines and drug components (Supplemental Fig. 1). Although we should emphasize that there are obvious variabilities between the two datasets (i.e., variation in FUT expression values) that should be taken into consideration which might be the result of different cell lines representing a cancer lineage, assay protocols, or culture media used. We can only argue that investigating the potential 
confounding roles of such factors is an avenue for a separate study. Regardless, the consistent correlation between FUT expression and drug sensitivity reiterates the findings of previous efforts that looked into the reproducibility and biological consilience between profiling data from GDSC and $\mathrm{CCLE}^{[32,33]}$.

Upon clustering of cell line-derived data into 30 cancer types, we determined univariate correlation between gene expression and a summary drug response measure (based on IC50 or AUC means). Spearman's correlation coefficient indicated that there is a variable but widespread association between fucosylation gene expression and drug resistance in both data sets (Fig. 1A and Supplemental Fig. 2A). Of interest in terms of its consistent high pancancer expression profile in both data sets is FUT8-notably the only enzyme-encoding gene known to directly mediate core fucosylation via $\mathrm{N}$-linkages ${ }^{[34]}$. To scrutinize whether the correlation between FUT8 expression and drug resistance is significantly cumulated in drugresistant cells, we categorized cell lines that are either sensitive or resistant based on the generalized drug response measurement and determined their correlation per class of drugs (Fig. 1B). Indeed, FUT8 broadly correlated with resistance to a variety of compounds but more strongly to inhibitors of receptor tyrosine kinase (RTK), epidermal growth factor receptor (EGFR), and insulin-like growth factor receptor (IGFR). Across all compound types, resistance to targeted therapies displayed the strongest correlation with FUT8 expression. Moreover, cell lines that contain mutations near or specifically at GDP-Fuc binding sites (resulting in amino acid change that eliminates or decrease fucosylation) in FUTs or other fucosylation genes collectively exhibited higher sensitivity to drugs (Fig. 1A and Supplemental Fig. 2, B and C).

In a separate analysis of the Cancer Therapeutics Response Portal (CTRP), a large-scale small molecule sensitivity data set, using the Computational Analysis of Resistance (CARE) scoring algorithm ${ }^{[35]}$, we showed that fucosylation gene expression displays significant correlation with resistance to kinase inhibitors (data on at least 84 drugs; Supplemental Fig. $1, D$ and $E$ ). In addition, using publicly available microarray and RNA-seq data, we found that high expressions of FUK, SLC35C1, and FUT8 are generally correlated with poor first progression or relapse-free survival (RFS) in various cancer patient cohorts (Supplemental Fig. 3).

Given that many of the target N-glycoprotein scaffolds of FUT8-mediated fucosylation are secreted $^{[34]}$, we next asked whether the association between fucosylation gene expression and drug resistance is interrelated with expression changes in the components of the core cancer secretome (CCS). Using defined component gene sets for CCS and protein glycosylation ${ }^{[8]}$, we observed coordinated pan-cancer increase or decrease of CCS and wideranging increase in expression of genes associated with glycosylation in general (Fig. 1C). It is important to note that the glycosylation gene set contains subsets of annotated gene classes involved in secretome glycosylation (i.e., FUTs, solute carriers, positive/negative regulators of glycosylation in the Golgi). To add resolution to this analysis, we also evaluated two of the largest glycosylation subsets in the dataset, protein $\mathrm{O}$ - and $\mathrm{N}$-linked glycosylation. Similarly, there is an extensive pan-cancer gene expression increase in both groups (Fig. 1C). Overlapping genes between CCS and glycosylation significantly correlated with resistance to both targeted the cytotoxic drugs, which may indicate that glycosylation of CCS components predicates drug sensitivity states.

Regulation of the DNA methylome influences the N-glycomes of the cancer secretome and plasma proteome ${ }^{[36,37]}$. Curious as to how promoter methylation of FUTs can associate with drug sensitivity, we analyzed the methylation status at $1 \mathrm{~kb}$ upstream of the transcription start sites (TSS) of each FUT (since this TSS proximal region often are loci for dense hyper- and hypo-methylation in cancer cell lines ${ }^{[38]}$ and queried drug sensitivity data in the GDSC. The overall fraction of FUT methylated loci varied across tumor types (Fig. 1D). As predicted, we observed significant negative correlation between FUT mRNA expression and promoter 
methylation. While the association between FUT methylation and drug sensitivity appears indiscriminately, FUT methylation profiles contradicted the correlation between FUT gene expression and resistance. In other words, cancer types exhibiting higher FUT methylation are more sensitive to targeted therapies with the exception of FUT1 and FUT6 (Fig. 1D), suggesting that cancer cells can inhibit fucosylation upon increased methylation of FUT promoter are more susceptible to therapy.

Based on our analysis, we hypothesized that response and resistance to targeted therapies involve the systemic regulation of core fucosylation of CCS components (Fig. 1E). We performed a potpourri of biochemical assays to characterize fucosylation in multiple cancer cell lines, cell secretomes, and patient sera and tissues. To enrich protein samples for core fucosylation, we used a lectin-conjugated bead capture strategy, where Aleuria aurantia lectin (AAL) served as the carbohydrate probe for core fucose (Fig. 1F; see Methods). Remarkably, lectin blotting revealed a distinct signature of enriched core fucosylation of serum proteins between 30 and $60 \mathrm{kDa}$ in lung cancer (LC) patients who received multiple cycles of osimertinib, a third-generation EGFR-tyrosine kinase inhibitor (TKI), compared to those of treatment-naïve patients (Fig. 1F and Supplemental Fig. 4). To quantitatively validate this result, we modified an $\mathrm{N}$-glycan oxidation assay originally developed to assess the activity of PNGases in releasing N-linked oligosaccharide chains from glycosylated scaffolds. These cleaved N-glycans, upon deamination by water, possess hemiacetal moiety at their reducing terminus that is highly reactive to water soluble WST-1, a tetrazolium salt dye that serves as an oxidation agent for N-glycans. In this reaction, WST-1 is converted to a formazan, producing a colorimetric readout (see Methods) ${ }^{[39,40]}$. Due to its simplicity, we decided to adapt and optimize this assay to quantify the release of $\mathrm{N}$-glycans from our samples using the glucoamidase PNGase F and glycosidases Endo S and F1.

Following analysis of in-gel excised 30 60 kDa serum proteins, PNGase F-released N-glycans showed significantly higher levels in osimertinib-treated patients compared to treatment-naïve patients (Fig. 1G), while this apparent difference was considerably moderated when N-glycans were released by either Endo S or F1. While PNGase F can cleave all N-glycans, we assumed that the glycans released from our samples are mostly those that contain core fucose (cleavage at $\alpha 1,6$ site) because the subjected $\mathrm{N}$-glycoproteins were captured using AAL (Fig. 1F). Thus, the reduction in detected $\mathrm{N}$-glycans released by Endo $\mathrm{S}$ or $\mathrm{F} 1$ reflects a specificity in cleaving different $\mathrm{N}$-glycans (at $\beta 1,4$ site) other than those containing core fucose. Note that Endo $\mathrm{S}$ has a high specificity for removing $\mathrm{N}$-glycans within the chitobiose core of native IgG while Endo $\mathrm{F} 1$ cleaves high mannose and some hybrid type $\mathrm{N}$-glycans ${ }^{[41,42,43]}$. The results potentially suggest that the cancer TIS from patients contains an elevated pool of core fucosylated proteins $<60 \mathrm{kDa}$.

To couple these results with an overall measure of fucosylation in various perturbation models, we developed a sandwich enzyme-linked lectin assay with varying affinities for AAL-captured fucosylated proteins (ELLA; Supplemental Fig. 5; see Methods). Using Ulex europaeus agglutinin I (UEA1)-AAL sandwich ELLA, we measured core fucosylation of cell-derived secretomes (Fig. 1H). TIS derived from cancer cells treated with targeted inhibitors of EGFR, $\mathrm{BRAF}$, and HER2 signaling unanimously led to an elevated secretome fucosylation (Fig. 11). To extend these findings to models of therapy resistance, we generated 16 stable drugresistant (DR) clones from various cancer types (lung adenocarcinoma, melanoma, and breast cancer) following stepwise evolution to appropriate targeted inhibitor pressures (Supplemental Fig. 6). All DR clone-derived secretomes showed increased fucosylation compared to secretomes derived from parental clones (Fig. 1I). Both 30 60 kDa TIS and secretome proteins from DR clones contained unanimously higher amounts of PNGase Freleased $\mathrm{N}$-glycans than those from DMSO or parental cell secretomes (Fig. 1J), while Nglycans released by either Endo S or F1 did not discriminate the amounts from all samples mirroring our observations from the patient sera. 
To substantiate this, we analyzed tissues from small cohorts of breast cancer (BC) and LC patients that received sequential multi-component therapy. Gene expression and enzyme activity analysis revealed that high expression of the fucose salvage pathway, FUT8, and SLC35C1 are strongly correlated with relapse (Supplemental Fig. 7, A and B). There was an immediate increase (16 h post-treatment) in Golgi-localized core fucosylation in drug-stressed LC and melanoma cells, and sustained activation in their respective DR clones (Supplemental Fig. 7C and Fig. 1K). We next profiled the expression of fucosylation genes in cancer cells with various oncogenic drivers upon apoptosis-inducing targeted therapy. While drug-induced expression changes varied between FUTs responsible for $\mathrm{O}$ - and $\mathrm{N}$-linked glycosylation, there was a marked increase in FUT8 and SLC35C1 expression (Supplemental Fig. 7D), all of which are associated with apoptosis (3-day treatment; Supplemental Fig. 7, E and F). In DR clones, both expressions are also amplified except with a pronounced fucose salvage pathway (Supplemental Fig. 7G). Because FUT8 is highly expressed in both drug-stressed cells and DR clones, we probed its potential role in therapy resistance. We first analyzed independent, genome-wide RNAi screening data from the Cancer Dependency Map (DepMap) project ${ }^{[44]}$, which houses pan-cancer genetic vulnerability maps. FUT8 is not classified as an essential gene in both sensitive and resistant cancer cell lines (Supplemental Fig. 8A), despite marginally higher essentiality scores in TKI-resistant cells than sensitive cells (Supplemental Fig. 8B). Regardless, treatment with EGFR-TKI or BRAFi and selection for resistance both led to higher FUT8-dependent GDP-Fuc catalytic activity (Supplemental Fig. 8C). Non-lethal concentrations of nine kinase inhibitors induced FUT8 expression while near-lethal concentrations moderately mitigated this effect (Supplemental Fig. 8D). We then used RNAi to functionally dissect the role of FUT8 upon targeted therapy. FUT8-targeting siRNAs augmented drug-induced cell killing and subsequent rescue was observed upon transfection with FUT8 cDNA (Supplemental Fig. 8E), all independent of cell proliferation (Supplemental Fig. 8F). We obtained similar results with SLC35C1 (Supplemental Fig. 8, G to I). These results are consistent with the idea that direct or indirect mediators of core fucosylation confer resistance to targeted therapies.

We next characterized fucosylation in cancer cell-derived secretomes to verify the differential secretome core fucosylation signature. Targeted kinase inhibition by EGFR-TKIs (gefitinib, erlotinib), HER2-TKI (lapatinib), or BRAFi (vemurafenib) induced fucosylation of secreted proteins $<60 \mathrm{kDa}$ (Fig. 1L). Similarly, secretomes derived from DR clones displayed an induced $<60 \mathrm{kDa}$ protein fucosylation (Fig. 1M). These results mimic the osimertinib-induced core fucosylation in LC patient sera. These are further accompanied by an overall increase in relapsed BC patient tissues and EGFR-TKI-treated LC patient sera (Supplemental Fig. 9, A and B). Well-known core fucosylated cancer biomarkers $\alpha$-fetoprotein (AFP) and $\alpha-1$ antitrypsin (A1AT), both >50 kDa, displayed systemic elevation in LC patient sera following osimertinib treatment and in secretomes of drug-stressed cells and DR clones, at least those expressing basal A1AT (Supplemental Fig. 9, C and D). Using molecular weight cut-off filtration, we confirmed that concentrated secreted proteins of $>30 \mathrm{kDa}$ from targeted inhibitortreated cells, their respective DR clones, and EGFR-TKI-treated LC patients display distinctively enriched fucosylation and core $\alpha-1,6$-linkages, but less so in $>100 \mathrm{kDa}$ pooled proteins (Fig. 1N and Supplemental Fig. 9E). Following targeted therapy, pooled $>30 \mathrm{kDa} N-$ glycoproteins from sensitive cells displayed increased release of fucosylated $\mathrm{N}$-glycans even at very low drug concentrations (from $0.001 \mu \mathrm{M}$ ), particularly in hypersensitive cell lines (Supplemental Fig. 9F). These can be controlled by FUT8 or SLC35C1, at least shown in vitro (Supplemental Fig. 9G).

Considering that some parental cell lines in our panel carry putative resistance drivers to specific TKIs, we sought to address two of the major 'off-target' resistance backgrounds in the context of targeted EGFR inhibition: MET amplification in H1993 cells and mutant KRAS activity in H358 cells. In $\mathrm{H} 1993$ cells and the derived GR clones, we evaluated the 
consequences on secretome N-glycosylation upon MET inhibition using RNAi (Supplemental Fig. 10A). MET knockdown sensitized both parental cells and GR clones to gefitinib (Supplemental Fig. 10B) but did not affect the overall secretome glycosylation even after gefitinib treatment (Supplemental Fig. 10C). However, TIS from gefitinib-treated H1993 cells displayed higher fucosylation and N-glycan release upon MET RNAi (Supplemental Fig. 10D). In H358 cells and the derived ER clones, we assessed the effects of selective loss of KRAS oncogenic addiction or targeting of the KRAS GTP/GDP-binding pocket on secretome Nglycosylation. To achieve such, we used two strategies: (1) an RNAi known to functionally inhibit oncogenic KRAS mRNA in cells that harbor mutations at codon 12 (i.e., G12C) ${ }^{[45]}$ (Supplemental Fig. 11, A to C), and (2) a KRAS agonist (KRA-533) known to promote accumulation of GTP-KRAS by prevention of cleavage from GTP into GDP ${ }^{[46]}$ (Supplemental Fig. 11D). Both strategies led to sensitization of both parental cells and ER clones to erlotinib (Supplemental Fig. 11, E and F) but did not affect the overall secretome glycosylation even after erlotinib treatment (Supplemental Fig. 11, G and H). Mimicking the MET RNAi results in H1993 models, only TIS from erlotinib-treated H358 cells displayed higher fucosylation and $\mathrm{N}$-glycan release upon mutant KRAS RNAi or KRA-533 treatment (Supplemental Fig. 11, I and J). While in both cases all DR clones were sensitized to the respective EGFR-TKIs, no overt changes were afforded in secretome fucosylation or N-glycan release. Our combined results support the idea that sensitizing parental cells by targeting putative resistancepromoting mechanisms produces a more reactive TIS with enriched fucosylation.

Taken together, our results suggest that targeted therapies induce a prevalent pan-cancer secretome core fucosylation that is primarily regulated by the fucose salvage-SLC35C1-FUT8 pathway and is enriched in the Golgi prior to secretion. This therapy-induced modification presumably is an evolvable mechanism towards establishing resistance.

\section{Therapy resistance via drug-induced secretome fucosylation.}

Limited tumor regression upon targeted therapy implicates that the microenvironment undergoes remodeling to critically sustain the remaining tumor population ${ }^{[7,47]}$. TIS, which consists of soluble mediators from this remodeled niche, predominantly promotes the survival and outgrowth of remnant tumor cells fostering subsequent disease relapse ${ }^{[7]}$. Considering that our data point to core fucosylation as a widespread PTM of the pan-cancer TIS, we proposed that de-N-glycosylation of the TIS prevents the outgrowth of residual DR tumor cells. To model a regressing tumor in vitro, we performed a multicolor homotypic 'one-pot' admixture assay by mixing a small percentage $(1 \%)$ of red-tracker-labeled DR clones with a large pool $(99 \%)$ of green-tracker-labeled sensitive cells in both 2D and 3D cultures. We then subjected these admixtures to targeted therapy, exogenously added PNGase $\mathrm{F}$ to de-N-glycosylate secretome proteins, and tracked the rebound of DR clones and regression of the sensitive cell pool (Fig. 2A). Following the formation of a 3D tumor spheroid, the population of the admixed gefitinib-resistant (GR) clone gradually expanded (observable after day 1 and steady from day 5), while sensitive cell population significantly decreased upon targeted therapy (Fig. 2B and Supplemental Movie, 1 and 2; representative PC9 admixture in Supplemental Movie 3). Addition of PNGase $F$ to these admixture secretomes led to a striking protein de- $\mathrm{N}$ glycosylation in culture (Fig. 2, C and D). Therefore, therapy-induced regression of mostly sensitive cells and population expansion of admixed minority GR and erlotinib-resistant (ER) clones are tightly linked with increased secretome core fucosylation (Fig. 2E).

Considering an abundant core fucosylation in all biologically active conditioned media (CM) occur before apoptosis or senescence and is enriched in the soluble secretome rather than apoptotic bodies, it is likely that cell-derived TIS and its $\mathrm{N}$-glycosylation are actively produced as a result of targeted oncogene inhibition (Supplemental Fig. 12). In both $3 D$ and $2 D$ admixture assays, secretome de-N-glycosylation blocked the growth acceleration of the DR clone promoted by TIS in various cancer backgrounds and targeted therapy settings (Fig. 2 , 
433 F and G; and Supplemental Fig. 13A), delayed the S-phase cycle of residual cell populations, 434 and promoted apoptosis (Fig. 2G and Supplemental Fig. 13, B and C). Consistently, in a CM co-culture assay, TIS stimulated the proliferation of low-density seeded DR clones while exposure to de-N-glycosylated TIS limited their outgrowth (Supplemental Fig. 13D). Of note, de-N-glycosylation in fresh media or DMSO CM did not affect DR clone proliferation (Supplemental Fig. 13D). At day 5, depletion of FUT8 or SLC35C1 efficiently blocked the expansion of DR clone population in a regressed cell admixture (Fig. $2 \mathrm{H}$ and Supplemental Fig. 13E), suggesting that the similar effect afforded by PNGase $\mathrm{F}$ is via protein de-Nglycosylation. In such a circumstance, we observed depletion of fucosylation (in both apoptotic bodies and soluble secretome) and intracellular kinase phospho-proteome (Fig. 2, I and J). We corroborated these in a CM co-culture assay (Fig. 2K), wherein de-N-glycosylated TIS prevented DR clones to form colonies and decreased kinase phosphorylation activity of EGFR, MET, and ErbB3, at least in GR and ER clones, respectively (Fig. 2, L and M). De-Nglycosylation by PNGase F in CM co-cultures (fresh media or CM from same cell/clone source) did not significantly influence the drug sensitivity of both sensitive cells and DR clones (Supplemental Fig. 14A), except in sensitive cells cultured in their own de-N-glycosylated TIS, where there is a widespread drug sensitization (Supplemental Fig. 14A). These point to the idea that fucosylation of the TIS from drug-treated sensitive cells is critical to its survivalenhancing effects not only on DR clones but also in drug-sensitive cells. Across all cell lines and DR clones, PNGase $F$ in-culture for up to 5 days did not affect cell proliferation (Supplemental Fig. 14B). We assumed that PNGase F in our cell admixture assays not only de-N-glycosylates secreted scaffolds but should also affect cell surface N-glycans. We inspected the potential changes on $\mathrm{N}$-glycosylation of cell membrane proteins in our admixtures by pooling subcellular fractions (admixture set-up as in Fig. 2A). At day 5, we only observed a significant increase in fucosylation from TIS and ER/Golgi fractions, not from cell membrane fractions, of EGFR-TKI-treated H1993 and PC9 admixtures (Supplemental Fig. $\underline{15}, A$ and $B$ ). In addition, there were no changes in the $<60 \mathrm{kDa}$ fucosylation signature in cell membrane fractions of the admixtures, unlike the significant increase in ER/Golgi fraction (Supplemental Fig. 15C). Regardless, PNGase F effectively de-N-glycosylated cell membrane proteins in-culture of both EGFR-TKI-treated H1993 and PC9 admixtures (Supplemental Fig. 15, D and E). Although we cannot completely rule out alternative possibilities, these results favor the idea that core fucosylation of the TIS, and not of membrane proteins, promotes the DR clone population expansion observed in our cell admixture experiments.

Next, we established a 'sequentially layered' 3D spheroid in vitro co-culture and monitored the growth of red fluorescent protein (RFP)-expressing DR clones (H1993-GR, PC9-ER) in the absence or presence of sensitive cells treated with kinase inhibitors or vehicle (Supplemental Fig. 16A). Resembling our initial findings, co-culture with EGFR-TKI (gefitinib or erlotinib)treated sensitive cells significantly promoted the growth of DR clones while the addition of PNGase F in the culture pronouncedly led to their growth retardation (Supplemental Fig. 16, $B$ and $C$ ). In these 3D admixtures at day 5, TIS de-N-glycosylation triggered the senescenceassociated secretory phenotype (SASP) and impeded the gene expression of factors previously described to promote resistant cell outgrowth in a regressing $\operatorname{TME}^{[7]}(\underline{\text { Fig. }} \mathbf{1 N})$. It appears that the response of DR clones in these admixtures upon TIS de-N-glycosylation is independent of fucosylation gene activity since there was no marked changes in expression (Supplemental Fig. 16D). Notably, long-term passaging and culture of DR clones in de-Nglycosylated TIS initiated a senescence response shown by strong senescence-associated $\beta$ galactosidase (SA- $\beta$-gal) activity, SASP activation, and arrested growth (Supplemental Fig. $\underline{17}$ ), elucidating the inhibited proliferative capacity of these clones in cell admixtures upon TIS de-N-glycosylation. These results demonstrate that the rebound of DR clone population in a model of tumor regression is dependent on fucosylated scaffolds of the TIS.

PON1 fucosylation is a critical feature of therapy-induced cancer secretomes. 
To identify relevant components of the TIS- and DR clone-specific N-glycomes, we performed label-free in-gel proteomics using AAL-captured 30 70 kDa secretome proteins derived from H1993 cells treated with or without gefitinib or the GR clone (Fig. 3A). Our analysis retrieved a fairly reproducible amount of peptide sequences per sample, which we used for downstream target identification (Supplemental Fig. 18A). Base peak chromatogram revealed differential mass ranges in all samples, with relatively higher overlapping similarity between gefitinibtreated cells and GR clone (Supplemental Fig. 18B). Because of the preliminary culture (i.e., $2 \%$ serum) and stress condition (drug treatment) requirements to produce TIS and semiquantitative nature of our screen, many proteins identified by this method are expected to be 'contaminants' derived from non-secreted apoptotic proteins, serum proteins (trypsin, albumin, keratin), uncharacterized proteins, immunoglobulins, and proteins below or above the range of excised in-gel sections ( $<30$ and $>70 \mathrm{kDa}$ ). As expected, we derived $>60 \%$ 'contaminant' protein coverage. We filtered these out and retained proteins that are only classified as 'secretory' or 'extracellular' based on the annotation criteria by UniProt (i.e., possession of Nterminal signal sequence), yielding a total of 57 unique, secretory-predicted proteins across the three conditions. Gene ontology (GO) analysis showed significant enrichment of biological processes (BPs) implicated in stress response, secretory pathway, and protein maturation in the ER/Golgi (Fig. 3B). Interestingly, BPs related to the metabolic regulation of oxidative stress were significantly overrepresented. Following selection of overlapping fucosylated secretome proteins between gefitinib-treated $\mathrm{H} 1993$ cells and GR clone, we identified 11 top hits using two different quantitative approaches [label-free quantification (LFQ) and intensity-based absolute quantification (iBAQ)]. Many of these hits are serum proteins described to have aberrant $\mathrm{N}$-glycosylation during cancer progression such as $\mathrm{AFP}{ }^{[24]}$ and the protein disulfide isomerase PDIA3 ${ }^{[48]}$.

Among the identified fucosylated proteins, we focused on PON1, an antioxidant enzyme, as its biological function matched the overrepresented BPs (Fig. 3C and Supplemental Fig. $\underline{\mathbf{1 8 C}}$ ). We previously identified PON1 to be systemically fucosylated in sera of late-stage metastatic small cell LC (SCLC) patients in an integrated glycoproteomics screen ${ }^{[49]}$. In gefitinib-treated H1993 cell secretome, we confirmed strong fucosylation of PON1, which appeared to have two isoforms: one with an apparent molecular mass of $\sim 55 \mathrm{kDa}$ while the other is $\sim 45 \mathrm{kDa}$ (Fig. 3D). Intracellularly, PON1 has both nuclear and cytoplasmic isoforms where a $\sim 40 \mathrm{kDa}$ cytoplasmic isoform is selectively enriched in LC patient tissues and cell lines ${ }^{[50]}$. To quantitatively validate secretome PON1 fucosylation in drug-stressed cancer cells and DR clones, we employed PON1 fucosylation-specific hybrid lectin ELISA (HLE; Fig. 3E). Despite different cell lineages, different oncogenic drivers, and different drugs, we found a widespread elevation of fucosylated secretome PON1 levels in multiple cancer cells upon targeted therapy (Fig. 3F). Similarly, PON1 fucosylation is enriched in secretomes derived from DR clones (at least those that have detectable PON1 gene expression) and is strikingly elevated in LC patient sera upon osimertinib treatment (Fig. $\mathbf{3}, \mathrm{G}$ and $\mathrm{H}$ ).

Using receiver operating characteristic (ROC) curves, we investigated whether fucosylated PON1 can discriminate between non-treated and osimertinib-treated LC patient sera. PON1 fucosylation discriminated against the conditions with high sensitivity and specificity with associated area under the curve (AUC) of 0.901, based on HLE measurements (Fig. 31, left). We previously reported that systemic serum PON1 is diminished in LC patients where fucosylated serum PON1 is increased (i.e., extensive disease). We hypothesize that this inverse relationship reflects an N-linked glycosylation-dependent control of PON1 activity. Supporting this idea, serum paraoxonase and arylesterase activities of PON1 were significantly differentiated between non-treated and osimertinib-treated LC patient sera (Fig. 3I, right and Supplemental Fig. 19A). Also, both of these enzyme activities significantly discriminated the treatment group with AUCs ranging from 0.76-0.89. In LC patient tissues, PON1 fucosylation is associated with relapse and discriminated it from non-relapsed LC with 
an AUC of 0.77 (Supplemental Fig. 19B). Next, we characterized intracellular PON1 fucosylation in DR clones. PON1 is primarily localized in the Golgi and has active fucosylation in the Golgi/ER fractions of GR and vemurafenib-resistant (VR) clones (Fig. 3, J and K). SLC35C1 RNAi significantly reduced Golgi-enriched PON1 fucosylation and promiscuously induced overall fucosylation in the nucleus of both DR clones (Fig. $\mathbf{3}, \mathrm{L}$ and $\mathrm{M}$ ), indicating a functional defect in the transport of GDP-Fuc along the secretory pathway.

To identify direct regulators of PON1 fucosylation, we examined CCLE-annotated PON1 Spearman's correlation and identified the cellular localization of each protein (Fig. 3N). Among the top 20 proteins, only PON3 and VWA7 showed co-localization with PON1 in the secretory pathway. We were intrigued by PON3, also a serum paraoxonase known to both preferentially interact and share numerous conserved PTM (i.e., N-glycosylation) sites with PON $1{ }^{[51]}$. In both GR and VR clones, there is an active co-localization between PON1 and PON3 (Fig. 30). While PON1 expression did not discriminate non-relapsed and relapsed BC patient tissues, high PON3 expression correlated well with relapse (Supplemental Fig. 19C). In addition, PON3 expression is increased in various drug-stressed cells and DR clones (Supplemental Fig. 19D). In Golgi/ER of H1993-GR, both PON3 and SLC35C1 RNAi, but not PON1 RNAi, inhibited PON1 fucosylation (Fig. 3P), demonstrating that PON3 directs PON1 fucosylation prior to secretion. Moreover, only PON1 RNAi, not PON3 or SLC35C1 RNAi, impeded Golgi/ER-specific paraoxonase activity (Fig. 3P), reflecting known differences between the two PONs in hydrolyzing paraoxon ${ }^{[52]}$. In a cross-linking GDP-Fuc activity assay, we showed that PON3 or SLC35C1 RNAi can ablate FUT8-directed transfer of fucose moiety from GDPFuc to N-glycan GIcNAc residue of PON1 (Fig. 3Q), implying direct functional regulation of PON1 fucosylation by PON3. Confirming the depletion of secretome PON1 fucosylation by a glycosidase, we showed that in the secretome, PON1 is de-N-glycosylated upon exogenous addition of PNGase $F$ onto cultures of sensitive cells and DR clones but without marked changes in GDP-Fuc activity on PON1 in Golgi/ER or PON1 secretion in sensitive cells, DR clones, and cells engineered to overexpress PON1 (Supplemental Fig. 19, E to G). All these suggest that core fucosylated PON1 is a major component of the constitutive $\mathrm{N}$-glycome of the cancer TIS and a signature of targeted therapy resistance.

\section{Core fucosylation enhances PON1 stability and prompts PON1 for secretion in therapy- resistant cancer cells.}

Given our prior knowledge on how systemic serum PON1 activity is diminished in LC patients and mouse model profiled with high serum PON1 fucosylation ${ }^{[49,50]}$, we hypothesized that therapy-induced protein glycosylation rewires the maturation steps of PON1 in the secretory pathway (Fig. 4A). PON1 has 23 predicted N-glycosylation sites with four Asn-X-Ser/Thr sequons-consensus amino acid sequences that determine core $\mathrm{N}$-glycosylation efficiency-all scored above the 'high potential' threshold (Fig. 4B). PON1 is predicted to be mostly folded and has a negative net electrical charge (-16 at pH 7). All four sequons of PON1 (N227, N253, $\mathrm{N} 270, \mathrm{~N} 324$ ) and their immediate vicinity have either neutral (0) or negative (ranging from 0.4 to -0.2 ) net charge (Fig. 4B). Among the four sequons, N324(GT) and N270(IS) are well conserved throughout species while N253(WT) is uniquely conserved in mammals (Fig. 4C). Both N253(WT) and N324(GT) sequons are located in the outer region of PON1's $\beta$-propeller structure while the other sequons are found in the innermost tunnel structure near the calciumbinding sites (Fig. 4D). Whether or not these indicate preference for aberrant glycosylation remain an open question. Regardless, the net charge, polarity, and X amino acid (in Asn-XSer/Thr) of sequons and their vicinity can generate preferable environments for aberrant protein N-glycosylation ${ }^{[53,54]}$.

To structurally map the bound N-glycans on PON1, we analyzed our previous tandem mass spectrometry (MS/MS) data ${ }^{[49]}$. We determined six aberrantly fucosylated glycans released 
from immunoprecipitated PON1, where $\mathrm{GlcNAc}_{2} \mathrm{Man}_{3}+\mathrm{HexNAc}_{2} \mathrm{Fuc}_{1}$ putative glycan structures are commonly present (Fig. 4E). Two of the most abundant glycans (peaks 1 and 2) were identified to have high FUT8 substrate specificity, while the rest (peaks 3 to 6 ) has either low specificity or not yet identified (Fig. 4E, bottom) ${ }^{[55,56]}$. To probe PON1 fucosylation in a site-specific manner, we introduced single-point mutation in two PON1 sequons [N253(WT) and N324(GT)]-predicted to display loss of N-glycosylation along with protein destabilization upon Asp $\rightarrow$ Gly mutation-and transfected the full-length wild-type (FL) or mutant constructs into sensitive cells, DR clones, and PON1-edited cells (Fig. 4F). Both PON1 mutants selectively reduced PON1 core fucosylation and prevented efficient GDP-Fuc transfer (Fig. 4, $\mathrm{G}$ and $\mathrm{H}$ ). Note that N253G displayed more robust effects than N324G. These mutants only had subtle effects on overall secretome $\mathrm{N}$-glycosylation and did not alter gene expression of PON1, PON3, and fucose salvage factors FUK, GMD, SLC35C1, and FUT8 (Supplemental Fig. 19, $\mathrm{H}$ and I). FL or PON1 mutants did not have significant effects on the response of sensitive cells to EGFR-TKIs while both N253G and N324G mutants, not FL, sensitized both GR and ER clones to EGFR inhibition (Supplemental Fig. 19J), suggesting that PON1 fucosylation is a resistance selected mechanism.

To validate the predicted effects of N253G and N324G mutations on PON1 stability, we assayed PON1 folding and synthesis upon protein cleavage by trypsin or de novo protein synthesis inhibition by cycloheximide (CHX) treatment. Immunoblotting of whole GR clone lysates revealed that $\mathrm{N} 253 \mathrm{G}$ remarkably promoted $\mathrm{PON} 1$ misfolding by completely sensitizing PON1 to cleavage by trypsin. N324G induced a noticeable PON1 cleavage only at a higher trypsin concentration (Fig. 4I). In the Golgi/ER of GR clone, similar effects were also afforded on PON1 when tested using ELISA and on protein glycosylation after PON1 immunoprecipitation (Fig. 4J and Supplemental Fig. 19K). In Golgi/ER of A549 cells, where there is basal PON1 expression, N253G did not alter PON1's sensitivity to trypsin. Conversely, the same mutation rendered PON1 from PON1-overexpressing cells sensitized to trypsin (Supplemental Fig. 19L). Furthermore, EGFR-TKI resistance or PON1 overexpression delayed the degradation of nascent polypeptides upon $\mathrm{CHX}$ treatment (Supplemental Fig. 19M). In GR clone, N253G had no significant effect on overall protein synthesis (Fig. 4K). In addition, EGFR-TKI resistance delayed the degradation of total fucosylated proteins and Golgi-specific PON1-immunoprecipitated glycoproteins (Supplemental Fig. 19, N and O). In GR clone, N253G accelerated the degradation of the lower $\mathrm{kDa}$ isoform of PON1, presumably its fucosylated form (Fig. 4L). More importantly, N253G significantly ablated PON1 secretion while N324G displayed a modest effect (Fig. 4M). Unexpectedly, N253G inhibited the intracellular arylesterase, but not paraoxonase, activity in GR clone (Supplemental Fig. 19P). This is consistent with our hypothesis that $\mathrm{N}$-glycosylation of PON1 governs its enzyme activity. Taken together, our data suggest that core fucosylation promotes PON1 stability prior to secretion in DR clones and PON1-overexpressing cells. This offers an answer to our longstanding question of how fucosylation affords a more stable, degradation-resistant PON1 state in the secretion, which seems to involve a rewired enzyme activity.

As an initial investigation of the functional consequences of PON1-specific core fucosylation inhibition on TIS-driven therapy resistance, we performed similar PON3 RNAi and PON1 sitedirected mutagenesis experiments in sensitive cells followed by $2 \mathrm{D}$ cell admixture assays. Inhibition of PON1 core fucosylation via PON3 silencing or PON1 N253G mutation in sensitive cells significantly prevented the population expansion of DR clones in regressed cell admixtures at day 5 (Supplemental Fig. 20), consistently supporting our hypothesis that PON1 core fucosylation is a critical and functional component of the cancer TIS.

Blockade of secretome core fucosylation confines therapy resistance via UPR effectors and a pro-inflammatory niche. 
DR clones in a regressing tumor model, we preliminarily mapped changes in 45 intracellular signaling pathways using a dual-luciferase activity reporter array. In a retrieved fraction of GR clone upon TIS de-N-glycosylation in 2D admixture, ER stress (ATF6), amino acid response (AAR element; ATF2, ATF3, ATF4), androgen receptor (AR) pathways, and E2F transcription were distinctively up-regulated, while stem cell factors (SOX2, NANOG, OCT4), interferonstimulated response (ISR element; STAT1, STAT2), STAT3, and hypoxia (HIF) signaling were selectively repressed (Fig. 5A). We validated this expression signature in 3D admixtures of DR clone (H1993-GR or PC9-ER) and sensitive cells (Fig. 5B and Supplemental Fig. 21A). In these admixtures, PON1-N253G transfection in sensitive cells phenocopied the effects of TIS de-N-glycosylation on intracellular signaling, senescence, regressing TME cues, kinase phospho-proteome, and growth of GR and ER clones (Fig. 5C and Supplemental Fig. 21, B to $G)$. These data point to a cascade of ER stress and UPR-regulated translational reprogramming events as mediators in blocking the growth of DR clones upon TIS de-Nglycosylation or fucosylated PON1 inhibition.

To probe these processes, we focused on ATF6, an ER-localized UPR-specific stress sensor ${ }^{[57]}$. In a CM co-culture, both TIS de-N-glycosylation and fucosylated PON1 inhibition actively induced Golgi/ER-localized ATF6 with enriched co-localization with fucosylated scaffold residues in DR clones (Fig. 5D and Supplemental Fig. 21H), which probably implicates ER stress-induced translocation/sorting of ATF6 from ER to Golgi. Next, we asked whether these stress-induced responses are orchestrated with oxidative stress given the phenotypic response of cell admixtures to inhibition of TIS-specific PON1 fucosylation via PON1-N253G mutation. Indeed, de-N-glycosylating TIS and inhibiting fucosylated PON1 in regressing cell admixtures markedly stimulated the production of reactive oxygen and nitrogen species (ROS/RNS), a hallmark of redox imbalance (Fig. 5E and Supplemental Fig. 21I). In DR clones co-cultured in modified TIS with scarce PON1 fucosylation, silencing ATF6 prohibited the generation of intracellular ROS/RNS (Fig. 5F and Supplemental Fig. 21J). In gefitinib-treated 3D cell admixtures, PON1-N253G-bearing sensitive cells restrained the growth of GR clone while ATF6 RNAi reverted this effect (Fig. 5, G and H). PON1-N253G promoted a pro-inflammatory environment in the same cell admixtures with increased levels of IL-6, TNF- $\alpha$, and GM-CSF cytokines in the secretome while ATF6 RNAi antagonized this induced cytokine signature (Fig. 5I).

To assess in more detail how perturbations in PON1 fucosylation influence the growth of DR clones, we established cell models to differentially modify secretome PON1 fucosylation. When overexpressed at a high degree (>700 fold) in cells without detectable PON1 (H460 and $\mathrm{H} 1993$ ), we observed an active PON1 fucosylation in the secretion. Stably knocking-out PON1 in wild-type PON1-expressing cells (A549) did not alter PON1 fucosylation intracellularly or in the secretion while stably knocking-out SLC35C1 in PON1-overexpressing cells led to suppression. Additionally, transfection of PON1-N253G construct or exogenous PNGase F treatment in PON1-overexpressing cells mitigated secretome PON1 fucosylation (summary in Fig. 5J). Accordingly, modified cells with de-N-glycosylated secretome PON1 have increased ROS/RNS and a more pro-inflammatory secretome (Fig. 5, K and L). More importantly, PON1 fucosylation-enriched TIS amplified the growth of GR and ER clones while cells with de-Nglycosylated PON1 prevented this growth coupled with increased intracellular caspase activities (Fig. 5, M and N). Furthermore, UPR target genes were consistently up-regulated in DR clones co-cultured with TIS or PON1-overexpressing cell secretomes with de-Nglycosylated PON1 (Supplemental Fig. 21K). In the same DR clones, restricted growth is associated with nuclear translocation of XBP1 (Supplemental Fig. 21L), indicating ATF6induced transcription factor activation.

Transcriptomics reveals resistance-associated genes mediated by secretome PON1 core fucosylation. 
To identify resistance-relevant genes that modulate responses to changes in secretome fucosylation, we performed a transcriptome-wide analysis of gene expression in DR clones at $48 \mathrm{~h}$ after co-culture with altered secretome conditions in vitro (Fig. 6A). The similarity in gene expression profiles was observed in replicate samples and among conditions that represent control ( $\mathrm{H} 1993$ and $\mathrm{H} 460)$, positive fucosylation regulation (gefitinib-treated $\mathrm{H} 1993$ and $\mathrm{H} 460-$ PON1), or negative PON1 fucosylation regulation (PNGase-treated and PON1-N253Gtransfected H460-PON1) (Supplemental Fig. 22A). Secretome de-N-glycosylation and PON1 fucosylation inhibition led to 433 altered gene expression, and pathway analysis revealed that overlapped genes were enriched for negative regulators of the cell cycle, and regulators of transcription and metabolism (Fig. 6B). The same conditions generated a more downregulated DR clone transcriptome associated with regulation of receptor signaling pathways, cell communication, and cell proliferation, among others (Fig. 6B). Enriched secretome fucosylation led to fewer differentially expressed genes that are mostly involved in cellular metabolic reactions (Supplemental Fig. 22B). Only one overlapping altered gene was detected between the two conditions promoting secretome fucosylation (gefitinib treatment and PON1 overexpression; Supplemental Fig. 22C). To identify molecular drivers of the DR clone's response to the suppression of secretome PON1 fucosylation, we integrated the data of differentially expressed genes in both PNGase F treatment and PON1-N253G transfection conditions. Secretome de-N-glycosylation led to 135 down- and 65 up-regulated genes, and secretome PON1 fucosylation inhibition resulted in 150 down- and 83 up-regulated genes, all with statistically significant $p$ values (Fig. 6C). 21 genes were up-regulated while 90 genes were down-regulated in both conditions (Fig.6D). This analysis highlighted C19orf25, RPS27L, CLDN2, PAQR3, and SOX4 as putative blockers; while THBS1, F3, TAGLN, ANKRD1, and DKK1 as positive regulators of secretome PON1 fucosylation-mediated DR clone outgrowth (Fig. 6E). To elucidate the implication of these genes in targeted therapy resistance, we analyzed genome-scale loss-of-function screening data from the Cancer DepMap project. We observed variable pan-cancer dependency signatures among the top 10 up- and downregulated hits from our initial screen and found that only C19orf25 is denoted as "commonly essential' in a pan-cancer ranking scheme. Regardless, many of these genes display high dependency scores in a fraction of cancer cell lines (Fig. 6F). We then examined whether these dependency profiles correlate with drug sensitivity. Indeed, many of these gene dependencies strongly correlate with either a drug-sensitive or a drug-resistant state to inhibitors of EGFR or RTK signaling, albeit varied $p$ values mainly due to different cancer lineages screened. Intriguingly, top overlapping up-regulated genes upon secretome de-Nglycosylation and PON1 fucosylation inhibition mostly correlate with a drug-sensitive state, while the down-regulated genes are more associated with resistance to EGFR-TKIs or RTK inhibitors (Fig. 6F). Validating these results, their pan-cancer expression profiles also correlated with broadly similar drug sensitivity signatures (Supplemental Fig. 23A). In large LC patient cohorts, high expression of two up-regulated gene hits, RPS27L and C19orf25, are correlated with increased first progression survival or RFS, while high expression of two downregulated gene hits, DKK1 and THBS1, are associated with poor survival outcomes after therapy (Fig. 6G and Supplemental Fig. 23B). Collectively, our data indicate that modulatory genes controlling DR clone response to inhibited secretome PON1 fucosylation are functionally associated with drug sensitivity to targeted therapies and are potential therapeutic targets to limit DR clone outgrowth.

\section{Core secretome fucosylation is engaged during metastasis and influences the dissemination of therapy-resistant cancer cells.}

Systemic aberration in fucosylation is implicated in multiple stages of metastasis ${ }^{[27,29,58]}$. Although it will be interesting to dissect in detail how TIS-specific core fucosylation is required in the dissemination and metastasis of DR clones, we limited our analysis with phenotypic correlations using mouse models, cell lines, and patient data in the context of therapy resistance and relapse to generate a conduit of preliminary data. First, we established an in 
vivo orthotopic model of lung metastasis using Lewis lung carcinoma (LLC) cells in C57BL/6 background mice and concurrently made xenograft tumors derived from the same cells for comparative analysis (Supplemental Fig. 24A). The majority of fucosylation gene expression is up-regulated in both LLC metastasized and xenograft tumors. However, we observed a distinct glycosylation signature (i.e., high FUT8 and low POFUT expression) in the metastatic lung nodules (Supplemental Fig. 24B). Note that several of these FUTs exert divergent effects during metastasis of different tumor cell types ${ }^{[29]}$. In melanoma, indirect transcriptional repression of FUK and reduced FUT1 expression promote distant metastasis and seeding capacities $^{[59,60]}$. These effects could also be extended to other malignancies such as pancreatic cancer and oral squamous cell (OSCC) and hepatocellular (HCC) carcinomas where tumors require reduced $\alpha(1,2)$ fucosylation for progression ${ }^{[29]}$. In our lung metastasis model, FUT1 and FUT2 expression-known mediators of $\alpha(1,2)$ fucosylation-were downregulated compared to both normal and xenograft tumor tissues (Supplemental Fig. 24B). Ex vivo biochemical analysis of both tissues and sera revealed aberrant core fucosylation immediately preceding large metastasis (in micrometastasized tumors) compared to normal tissue or xenograft tumors (Supplemental Fig. 24, C and D).

Whereas the contribution of TIS to the metastatic outgrowth of remnant DR clones in regressing tumors has been previously investigated, almost nothing is known about the role of TIS fucosylation in mediating such process. To address this in vitro, we set up transwell invasion and monolayer gap-closing assays and used PNGase F to de-N-glycosylate cell TIS or LLC mouse serum (schematic in Supplemental Fig. 24E). Indeed, TIS or serum from mouse burdened with lung metastasis increased the invasion of both GR and ER clones. Notably, there was a marked reduction in their invasion upon protein de-N-glycosylation (Supplemental Fig. 24E). In a gene expression analysis of recovered invasive DR clones in the same co-culture conditions, we observed suppression of mesenchymal and cancer stem cell (CSC) phenotypes (Supplemental Fig. 24F). In addition, the same protein de-Nglycosylation conditions inhibited gap-closing migration of DR clones (Supplemental Fig. 24G). Substantiating these findings, high degree fucosylation is associated with BC relapse, lymph node metastasis, and HER2 expression in a small cohort of patients (Supplemental Fig. 24H). Furthermore, FUT8 expression significantly correlated with CSC expression in BC tissues from the same patient cohort (Supplemental Fig. 24l). Note that higher expressions were observed in relapsed tumors. In a much larger patient cohort (METABRIC dataset), amplification of fucosylation genes FUK, SLC35C1, and FUT8 are significantly associated with CSC gene expression (Supplemental Fig. 24J).

Lastly, we aimed to uncover a potential regulatory role for PON1 fucosylation in metastasis. We engineered LLC cells to overexpress PON1 and found a striking increase in secretome PON1 fucosylation (Supplemental Fig. 24, K and L). Using these PON1-modified cells (LLCPON1) along with control cells derived from the same parent cell line (LLC-CC), we interrogated their metastasis to the lung (Supplemental Fig. 24M). LLC-PON1 cells displayed higher metastatic propensity, tissue and serum core fucosylation and serum PON1 fucosylation than LLC-CC cells in vivo (Supplemental Fig. 24, $\mathrm{N}$ to $\mathrm{R}$ ). In metastasized lung nodules, LLC-PON1 displayed CSC and N-glycosylation-specific gene expression signatures (Supplemental Fig. 24S). Although further mechanistic work will be needed, our data suggest that metastasis is associated with an aberrant N-glycome-with elevated PON1 fucosylation as a critical component-that serves as a niche for developing therapy resistance.

\section{DISCUSSION}

Here we identify a distinct $\mathrm{N}$-glycome signature of the pan-cancer TIS. Using complementing fucosylation enrichment and detection approaches, we show that an induced secretome $<60$ $\mathrm{kDa}$ protein fucosylation is systemically aberrant in cancer cells and patients upon targeted therapy. This modification appeared to be selected during resistance evolution as cell-derived 
DR clones and relapsed cancer patients display the same secretome aberration. Counterintuitively, TIS marks both response and resistance to targeted therapy ${ }^{[7,47]}$. Subsequent regression of tumors and their TME in response to targeted therapy lead to the release of TIS that feeds the outgrowth of minority DR clones and survival of other cellular components (i.e., stromal cells) of the targeted microenvironment ${ }^{[7,47]}$. We reveal that core fucosylation of the TIS augments this effect. De-N-glycosylating the TIS by a glycosidase suppressed critical resistance-mediating survival cues and promoted a senescent state in regressing cell admixtures. Thereby glycans bound to $\mathrm{N}$-glycosylated scaffolds of the TIS, not the released $\mathrm{N}$-glycans per se, are required to establish a resistance-conferring niche. Mechanistically, directly blocking the transport of GDP-Fuc into Golgi or transfer of fucose onto proteins prevent the population rebound of remnant DR clones, encouraging a more drugresponsive cell population.

823
To date, there are 11 FUTs and two POFUTs known to catalyze fucose transfer from donor GDP-Fuc to various acceptor scaffolds such as glycoproteins and glycolipids, following Golgispecific transport of GDP-Fuc by SLC35C $1^{[27,28,29]}$. These enzymes can compete in a mutually exclusive fashion to synthesize glycans in the Golgi and are exploited during tumorigenesis. While previous studies have implicated FUTs in multidrug resistance in several cancer types ${ }^{[29]}$, there currently has no systemic analysis that describes the degree and scope of this connection. Mapping the pan-cancer pharmacogenomic profiles of these FUTs and SLC35C1 revealed that their expression broadly correlated with resistance to multiple targeted therapies while their inhibitory cues (i.e., promoter methylation and GDP-Fuc binding site mutations) are widely associated with a drug-sensitive state. We show that the FUK-SLC35C1-FUT8 core fucosylation axis is significantly correlated with patient relapse in both large and small patient cohorts. This fucose metabolism pathway appears to be a pre-requisite in driving our observed secretome fucosylation in drug-stressed cells and DR clones. High degree pan-cancer expression of FUT8 and its activity in the Golgi entail the substrate specificity of FUT8 in fucosylating scaffolds in the secretory pathway. In a much broader context, we corroborated this by showing that the gene set encoding for CCS components contains a subset of glycosylation genes that display increased expression signature. We reveal that FUT8 or SLC35C1 can directly regulate the distinct $<60 \mathrm{kDa}$ secretome fucosylation specifically in DR clones but not in sensitive cells. We note that $>100 \mathrm{kDa}$ secretome fucosylation can effectively be mediated by either of the two factors in sensitive cells, uncovering differential target processing of core fucosylated products prior to their secretion.

Our discovery of PON1 fucosylation as a component of the pan-cancer TIS contextualizes its systemic regulation in cancer patients upon therapy. Our previous study along with others suggests a compelling serological biomarker potential for fucosylated PON1 in advanced SCLC and early HCC ${ }^{[49,61,62]}$. While it is conceivable that overabundance of fucosylated PON1 in the secretion is due to overacting FUTs (i.e., FUT8) and fucose metabolic reactions in the liver, it does not provide an intuitive explanation for reduced serum PON1 level and restricted enzyme activity in multiple cancer patients and mouse models profiled previously ${ }^{[49]}$ and in this study. This lack thereof has led us to examine how fucosylation influences the stability of PON1 prior to its secretion. Our data show that core fucosylation at a sequon located in the terminal region of the arylesterase domain, a conserved site among mammals, determines PON1 stability and assures proper folding prior to the secretion of PON1 from DR clones or PON1-overexpressing cells. This indicates that induction of core fucosylation pathway rewires the maturation (i.e., folding) of PON1 along the secretory route, generating a more degradation-resistant PON1 with altered enzyme activity. We speculate that in disease states where there is an abundant serum fucosylation, non-fucosylated or less fucosylated PON1 cannot persist longer because of proteolytic insults in the blood. Among the three-member PON family, PON1 and PON3 are both secretory antioxidants bound to high-density lipoprotein (HDL) and share considerable structural homology ${ }^{[51,52]}$. Our data revealing PON3mediated PON1 fucosylation in the Golgi hence establishes an altered Golgi redox 
homeostasis prior to the secretion of proteins. Indeed, our pathway-focused screen reveals that defective secretome PON1 fucosylation in the TIS promotes the expression of transcription factors that regulate response to oxidative stress and pro-inflammatory niche, and repression of hypoxia in a suppressed DR clone. Concurrently, our transcriptome-wide analysis demonstrates that genes negatively regulating response to stimulus and cell communication act as modulators upon inhibition of secretome PON1 fucosylation. Thus, targeted strategies to control them might limit therapy resistance.

Metastasis of DR clones requires TIS-derived signals to spur eventual relapse after therapy ${ }^{[7}$, metastasis of several cancers ${ }^{[27,28,29]}$. In melanoma, a systems biology approach identified that transcriptional activation of FUT8 drives metastasis-distinct core fucosylation as opposed to $\alpha 1,2$-fucosylation in primary tumors ${ }^{[58]}$. While it remains largely unknown how systemic aberration in core fucosylation of serum proteins could influence specific steps in the metastatic cascade, several metastatic phenotypes have been linked with overacting core fucosylation. For example, FUT8 activity and TGF- $\beta$ receptor fucosylation transduce downstream effectors of the epithelial-to-mesenchymal transition (EMT) eliciting metastasis. Moreover, many cancer stem cell (CSC) markers are glycoproteins (i.e., CD44, CD133) that display differential glycosylation during metastasis ${ }^{[63,64]}$. Our data argue that increased serum fucosylation immediately precedes lung micro-metastases and is highly aberrant in large, latestage metastases, accompanied by high expression of FUT8 in metastasized lung tumors. This LC metastasis-specific serum fucosylation stimulated the invasion and migration of DR clones by promoting EMT and CSC gene signatures in vitro. Additionally, LC tumors with high PON1 fucosylation profile displayed higher metastatic propensity than primary tumors. These findings indirectly support the idea that TIS fucosylation promotes the dissemination of residual DR tumor cells.

To this end, we report an aberrant signature of secretome core fucosylation functionally associated with multi-targeted therapy resistance in different cancer lineages. Our study highlights the fucosylation of PON1 as a component of a complex, reactive secretome induced upon targeted therapy and in turn stimulates resistance. This proof-of-concept study underscores new insights into the biological basis of cancer recurrence. We acknowledge that while our findings are all reproducible, they still require further validation, perhaps using patient-derived animal models. Regardless, the generality of our findings implicates that targetable aberration in secretome fucosylation and modulatory factors controlling response to this niche should be considered in managing clinical cancer relapse.

\section{MATERIALS AND METHODS}

\section{Data reporting and statistics}

No statistical methods were used to predetermine sample size. The experiments were not randomized unless otherwise stated. The investigators were not blinded to allocation during experiments and outcome assessment. All quantitative data are presented as means \pm SD unless otherwise specified. Student's $t$, Mann-Whitney, Dunnett's, Wilcoxon rank-sum, Kruskal-Wallis, Mantel-Cox and chi-squared tests; and ROC analyses were performed with GraphPad Prism 8.4. The number of samples or biological replicates $(n)$ is indicated in each figure panel. For bioinformatics, all adjusted $p$ values $\left(p_{\text {adj }}\right)$ were adjusted to control for the false discovery rate (FDR) using the Benjamini-Hochberg procedure. Statistical significance was defined as $p<0.05$.

\section{Human cancer patient samples and ethics statement}

All human blood and tissues from three cohorts of patients diagnosed to have lung 
adenocarcinoma or squamous cell carcinoma or breast carcinoma were collected and analyzed with approved protocols in accordance with the ethical requirements and regulations of the Institutional Review Board of Seoul National University Hospital after securing written informed consent (IRB Nos. 1104-086-359 and B-1201/143-003). All samples were selected and categorized randomly. Patients underwent surgical resection of their primary or metastatic tumors at Seoul National University Hospital. Tissue and blood samples were obtained by core needle biopsy. In the first cohort, 14 samples of plasma and 30 samples of sera from treatment-naïve and osimertinib-treated NSCLC patients with EGFR-activating mutations were obtained in a routine diagnosis. In the second cohort, 53 paired lung cancer tumor tissues and adjacent normal tissues were obtained during surgery. Preoperative chemotherapy was not conducted on all patients in this cohort. In the third cohort, 33 breast cancer tissue samples were obtained during surgery. Patients received primary systemic therapy (PST) and adjuvant chemotherapy. Pathological complete response following PST was defined as complete disappearance of all invasive cancer or only residual ductal carcinoma in situ. In all cohorts, post- or preoperative radiation therapy was not performed. Blood and tissue processing and histopathological data interpretation were overseen by expert pathologist co-authors (H.S.R., S.C., T.M.K.). Clinicopathologic information from three patient cohorts was abstracted from medical records and de-identified as shown in Supplemental Tables 1, 2 , and $\underline{\mathbf{3}}$. Source DNAs and RNAs were extracted from archived Formalin-Fixed Paraffin-Embedded (FFPE) tumor and adjacent normal tissues. Lysates were obtained from frozen tumors. Frozen samples were "snap-frozen" in liquid nitrogen and stored at $-80^{\circ} \mathrm{C}$. For plasma collection, samples were centrifuged at $1,600 \mathrm{~g}$ for $10 \mathrm{~min}$ within an hour of the blood draw, then an additional centrifugation of $20,000 \mathrm{~g}$ for $10 \mathrm{~min}$ was carried out. For serum collection, blood was allowed to clot for 15-30 min at room temperature (RT) prior to the same centrifugation. All aliquots were stored at $-80^{\circ} \mathrm{C}$. Each aliquot was thawed no more than twice prior to use. Multiple Affinity Removal System (MARS) HSA/lgG spin columns (Agilent) were used to deplete albumins and IgGs from blood samples. Depleted samples were concentrated using Amicon Ultra-2 mL Centrifugal Filters [Merck; 3k molecular weight cut-off (MWCO)] according to manufacturer's instructions.

\section{Cell lines}

Human H292, H1993, H358, HCC4006, H460, H1299, and A549 cell lines [American Type Culture Collection (ATCC) nos. CRL-1848, CRL-5909, CRL-5807, CRL-2871, HTB-177, CRL5803, and CCL-185, respectively; obtained in 2014 to 2016] were grown under standard conditions in RPMI 1640 (Welgene) supplemented with 10\% fetal bovine serum (FBS) alternative Fetalgro bovine growth serum (RMBIO) or EqualFETAL bovine serum (Atlas biologicals), $2 \mathrm{mM} \mathrm{L-glutamine,} \mathrm{and} \mathrm{penicillin} \mathrm{(100} \mathrm{U/ml)-streptomycin} \mathrm{(100} \mathrm{\mu g/ml;} \mathrm{Invitrogen).}$ PC9 and HCC827 [originally provided by J. K. Rho (Asan Medical Center, University of Ulsan, Seoul, Korea)], EBC-1 [Japanese Collection of Research Bioresources (JCRB) Cell Bank no. JCRB0820], HCC78 [German Collection of Microorganisms and Cell Cultures (DSMZ) GmbH no. ACC563], H3122 [originally provided by P. A. Jänne (Dana-Farber Cancer Institute, Boston, MA, USA)], and SKBR3 [originally provided by D. M. Helfman (KAIST, Daejeon, Korea)], all obtained in 2017, cell lines were grown in RPMI 1640 with the same supplementation as mentioned above. Human H1975, H2009, A375, HEK-293T cell lines [ATCC nos. CRL-5908, CRL-5911, CRL-1619, CRL-3216; obtained in 2017] were grown in Eagle's minimum essential medium (Merck), DMEM/F12 (Gibco), and DMEM (Welgene) with the same supplementation as mentioned above except without additional L-glutamine and contained in addition $1 \mu \mathrm{g} / \mathrm{mL}$ amphotericin B. Mouse LL/2 (LLC1; ATCC no. CRL-1642; obtained in 2015) was grown in BME with Earle's salts (Merck) with the same supplementation as mentioned above. All cells were grown in a humidified incubator at $37^{\circ} \mathrm{C}$ with $5 \% \mathrm{CO} 2$ and were tested regularly for mycoplasma contamination. All cell lines used were negative for mycoplasma (Cosmogenetech mycoplasma test service). 


\section{Drug-resistant clones}

To generate DR clones, sensitive cell lines were seeded at low density and continuously exposed to gradually increasing concentrations of the drug for at least 12 weeks and for as long as $>52$ weeks. All clones were derived and expanded from colonies and maintained at specific drug concentrations. Clones were passaged every 2 or 3 days with adding fresh drug concentration. Characterization of resistance is summarized in Supplemental Fig. 6A.

\section{Cell secretomes preparation}

To generate secretomes, $3 \times 10^{6}$ sensitive cells and $7 \times 10^{6}$ DR clones were plated on $15-\mathrm{cm}$ plates in standard media and allowed to adhere overnight. The media was then replaced with fresh media with $2 \%$ dialyzed FBS and indicated drugs for $48 \mathrm{~h}$. FBS was dialyzed in-house (against $0.15 \mathrm{M} \mathrm{NaCl}$ until glucose reached $<5 \mathrm{mg} / \mathrm{dL}$ ) using $10 \mathrm{k} \mathrm{MWCO}$ dialysis tubing (Fisher Scientific) at $4^{\circ} \mathrm{C}$ for $6 \mathrm{~h}$. Secretomes were centrifuged at 1,000 r.p.m. for 5 min, vacuum filtered using $0.45 \mu \mathrm{m}$ cellulose acetate membranes (Whatman), and immediately placed on ice. For 2D co-culture, secretomes were stored at $4^{\circ} \mathrm{C}$, warmed prior to use, and were used only within $48 \mathrm{~h}$. For 3D co-culture, only freshly prepared secretomes were used and were further concentrated using Amicon Ultra-15 mL Centrifugal Filters (3k MWCO). For biochemical assays, secretomes were further concentrated using Amicon Ultra-15 mL Centrifugal Filters (3k, 10k, 30k, 50k, 100k MWCO as indicated) and depleted of albumins and IgGs using MARS HAS/lgG spin columns. Aliquots were "snap-frozen" in liquid nitrogen and stored at $-80^{\circ} \mathrm{C}$ until use. Aliquots were thawed only once.

\section{$\mathrm{N}$-glycosylation/fucosylation assays}

For enrichment of core fucosylated proteins/lipids, we used AAL as a probe to capture scaffolds with bound fucose linked $(\alpha 1,6)$ to $\mathrm{N}$-acetylglucosamine or fucose linked $(\alpha 1,3)$ to $\mathrm{N}$ acetyllactosamine related structures. We note that $A A L$ also reversibly binds fucose attached to nucleic acids. Bio-spin columns (Bio-Rad) were packed with $1.5 \mathrm{~mL}$ agarose bead-bound AAL (Vector Laboratories). Agarose beads were initially maintained in an inhibiting solution [10 mM HEPES (pH 7.5), $0.15 \mathrm{M} \mathrm{NaCl}, 10 \mathrm{mM}$ fucose, $0.04 \% \mathrm{NaN3}$ ] at $4^{\circ} \mathrm{C}$. Concentrated secretomes $(500 \mathrm{uL})$ or sera/plasma $(40 \mathrm{uL})$ were thawed at $4^{\circ} \mathrm{C}$ in ice, diluted in $1.5 \mathrm{~mL} \mathrm{AAL}$ adsorption buffer (AffiSpin-AAL kit; GALAB), incubated in ice for $5 \mathrm{~min}$, loaded onto packed bio-spins, and incubated at $4^{\circ} \mathrm{C}$ for at least $12 \mathrm{~h}$. Unbound proteins/lipids were removed by flow-through (only by gravity) and washing with adsorption buffer and PBS. Fucosylated proteins were eluted twice with $50 \mathrm{uL} \mathrm{AAL}$ elution buffer B1 or $40 \mathrm{uL}$ glycoprotein eluting solution for fucose-binding lectins (Vector Laboratories) at $4^{\circ} \mathrm{C}$ for $1 \mathrm{~h}$ per round. Remaining bound fucosylated proteins were forcedly eluted. Samples were scaled-up to produce at least $80 \mathrm{uL}$ eluted proteins. All reagents and columns were pre-chilled in ice prior to use. Eluted proteins were precipitated using the trichloroacetic acid (TCA)-sodium deoxycholate (DOC) method as described previously with minor modifications ${ }^{[65]}$. Protein concentrations were measured using the Bradford reagent (Bio-Rad).

We developed a sandwich ELLA assay to quantify fucosylated proteins in AAL-enriched samples (Supplemental Fig. 5). 96-well microtiter plates (Koma Biotech) were coated with $0.4 \mu \mathrm{g}$ native, unconjugated MAL II, SNA, LCA, BTL, PSA, UEA1, ConA, or RCA1 lectins (Vector Laboratories) in $100 \mathrm{uL}$ coating buffer (15 mM Na2CO3, $35 \mathrm{mM} \mathrm{NaHCO}, 0.02 \%$ $\mathrm{NaN3}, \mathrm{pH} 9.6$ ) at $37^{\circ} \mathrm{C}$ for $2 \mathrm{~h}$. The plates were additionally incubated with $0.1 \mathrm{~mL}$ oxidation buffer $(20 \mathrm{mM}$ NalO4) per well. Lectin solution was removed by three washes with PBSTween-20 (0.05\%; PBST). Plates were then blocked with 3\% bovine serum albumin (BSA) in PBST for $1 \mathrm{~h}$ at RT. Concentrated secretomes, lysates, or sera/plasma were added to each well and incubated at RT for $2 \mathrm{~h}$. The plate was gently washed three times with PBST to remove unbound proteins. $100 \mathrm{uL}$ of $4 \mu \mathrm{g} / \mathrm{mL}$ biotinylated AAL (Vector Laboratories) was 
added and incubated at RT for 90 min. Lectin solution was removed and HRP-conjugated streptavidin (Biolegend) was added and incubated at RT for 90 min followed by two additional washes with PBST. 1-Step Turbo TMB-ELISA substrate solution (Thermo Scientific) was used for detection. Absorbance was measured at $450 \mathrm{~nm}$ in a microplate reader (VersaMax, Molecular Devices).

For N-glycan release assay, we optimized a previously described protocol to quantify glycosidase-induced release of $\mathrm{N}$-glycans ${ }^{[39]}$. Briefly, $20 \mathrm{uL}$ concentrated samples were mixed with $2.5 \mathrm{uL}$ sodium phosphate or citrate buffer $(500 \mathrm{mM}, \mathrm{pH} 7.5)$ and $10 \mathrm{uL}$ total $8 \mathrm{U}$ PNGase $\mathrm{F}$ or $10 \mathrm{U}$ Endo $\mathrm{S} / \mathrm{F} 1$ and incubated at $37^{\circ} \mathrm{C}$ for $12 \mathrm{~h}$ in a humidified chamber and heatquenched at $95^{\circ} \mathrm{C}$ for $5 \mathrm{~min}$. Reactions were then mixed with $20 \mathrm{uL} 2.5 \mathrm{M}$ TCA solution, vortexed for $5 \mathrm{~min}$, and centrifuged at $12,000 \mathrm{~g}$ for $30 \mathrm{~min}$. $15 \mathrm{uL}$ supernatants were mixed with $7.5 \mathrm{uL}$ of $4 \mathrm{M} \mathrm{NaOH}, 12.5 \mathrm{uL} 1.7 \mathrm{mM}$ aqueous WST-1 solution, and incubated for $1 \mathrm{~h}$ at $50^{\circ} \mathrm{C}$. For in-gel N-glycan release, in-gel proteins were trypsin digested overnight (see details below). Samples containing extracted peptides were reduced in a SpeedVac until at least 10 $\mathrm{uL}$ was reached. Reduced samples were mixed with $10 \mathrm{uL} \mathrm{H} 2 \mathrm{O}$ and subjected to the same protocol as mentioned above. Absorbance was measured at $584 \mathrm{~nm}$. The amount of released $\mathrm{N}$-glycans were quantified using maltose (Sigma), an N-glycan mimic in this assay, as an external standard.

AAL blotting was performed as described previously ${ }^{[49]}$. Briefly, AAL-enriched precipitated samples (10-15 $\mu \mathrm{g}$ concentrated cell secretome or 3-5 $\mu \mathrm{g}$ of sera/plasma proteins) were subjected to $12 \%$ SDS-PAGE. The gels were transferred to nitrocellulose membranes (Whatman). The membranes were blocked with $1 \mathrm{x}$ Carbo-free blocking solution (Vector Laboratories) at $4^{\circ} \mathrm{C}$ for at least $2 \mathrm{~h}$ and incubated with $5-20 \mu \mathrm{g} / \mathrm{mL}$ of biotinylated AAL at RT for $1 \mathrm{~h}$. Membranes were washed three times with PBST, incubated with HRP-conjugated streptavidin at RT for $1 \mathrm{~h}$, washed three times with PBST, and developed using an ECL system (Amersham).

Glycoprotein staining of SDS-PAGE gels was performed using the GelCode glycoprotein staining kit (Pierce) according to manufacturer's protocol. Stained glycols appear as magenta/pink bands.

For HLE of target protein fucosylation, we modified a previously described protocol using an ELISA starter kit (Koma Biotech) ${ }^{[49]}$. Briefly, 96-well microtiter plates were coated with $120 \mathrm{ng}$ PON1 (18155-1-AP, Protein Tech), AFP (ab3980, Abcam), or A1AT (ab9399, Abcam) monoclonal antibodies in $100 \mathrm{uL}$ coating buffer at $37^{\circ} \mathrm{C}$ for $3 \mathrm{~h} .100 \mathrm{uL}$ of oxidation buffer was added per well for 30 min and blocked with $3 \%$ BSA in PBS for $2 \mathrm{~h}$ at RT. The plates were washed four times with PBST. All AAL-enriched samples were diluted 10-fold in PBS, $100 \mathrm{uL}$ of each sample was added to each well, and incubated at RT for $2 \mathrm{~h}$. After multiple washes with PBST, $2 \mu \mathrm{g} / \mathrm{mL}$ biotinylated AAL was added and incubated at RT for $90 \mathrm{~min}$. Lectin solution was removed and HRP-conjugated streptavidin (Biolegend) was added and incubated at RT for 90 mins followed by two additional washes with PBST. A 1-Step Turbo TMB-ELISA substrate solution (Thermo Scientific) was used for detection. Absorbance was measured at $450 \mathrm{~nm}$.

For lectin fluorescent staining of cells and paraffin sections, we used $15 \mu \mathrm{g} / \mathrm{mL}$ fluoresceinlabeled AAL (Vector Laboratories) or $4 \mu \mathrm{g} / \mathrm{mL}$ FITC-conjugated UEA1 (Thermo Scientific) according to manufacturer's protocol and following standard immunofluorescence protocols.

GDP-Fuc activity of FUT8 was assayed using GDP-Glo glycosyltransferase assay kit (Promega) following manufacturer's protocol. Luminescence was read on luminometer (POLARstar Omega). 
For in-culture and exogenous secretome/serum de- $\mathrm{N}$-glycosylation, $10 \mu \mathrm{g} / \mathrm{mL}$ recombinant PNGase F (9109-GH, R\&D Systems; 36405.01, SERVA) and 8U PNGase F (P0704L, NEB; P7367, Sigma) were used, respectively, unless otherwise specified, for indicated times described in each figure description. PNGase F was not removed in any of the in-culture experiments for indicated incubation periods, except in Supplemental Fig. 14A where PNGase F-treated media was replaced with drug-containing fresh media for drug sensitivity assay. Protein/lysate sample de-N-glycosylation using PNGase F (NEB), Endo S (NEB), or Endo F1 (Sigma) was performed following manufacturer's protocol with slight modifications on incubation period.

\section{Cell tracking experiments}

To fabricate a 3D tumor spheroid array, polydimethylsiloxane (PDMS)-based positive master mold with an array of 225 spherical microwells $(15 \times 15)$ was prepared as we previously described $^{[66]}$. The mold was immersed in $70 \%(\mathrm{v} / \mathrm{v})$ ethanol and sterilized for $30 \mathrm{~min}$ in UV before use. Agarose powder (LPS solution) was added to RPMI 1640 at a concentration of $3 \%$ $(\mathrm{w} / \mathrm{v})$ and heated for a short time to dissolve completely. Before gelation, the fully-melted agarose solution was poured in a $35-\mathrm{mm}$ cell culture dish (3 $\mathrm{mL} /$ dish; SPL) and the sterilized master mold was immediately inserted into the gel solution to create the microwells. After the agarose was solidified at RT for 20 min, master mold was gently removed. PBS ( $3 \mathrm{~mL} / \mathrm{dish})$ was added to the agarose-based microwell to keep them hydrated before use. Cell admixtures seeded into these microwells can immediately form spheroids (Supplemental Movie 1 and Fig. 2B). Cell line variability in the number and size of spheroids is observed per well (Supplemental Fig. 6D).

For tracking experiments in cell admixtures, sensitive cells were labeled with CellTrackerGreen (CMFDA) while DR clones with CellTracker-Red (CMTPX) or -Deep Red (Thermo Scientific) or transfected with pCAG-LifeAct-RFP (Ibidi) according to manufacturer's protocol. RFP-labeled clones were stably selected using geneticin (Thermo Scientific) following manufacturer's instructions. Labeled cells and clones were filtered using a cell strainer (Merck) and seeded either as 'one pot' or 'sequential layer' 2D and 3D admixtures as detailed (Fig. 2A and Supplemental Fig. 16A). 3D admixtures were live imaged while 2D admixtures were fixed with $3.7 \%$ formaldehyde in PBS for 5 min and washed with PBS prior to imaging. Live imaging of 3D tumor spheroids was performed using a fluorescence inverted microscope (Nikon Eclipse Ti) equipped with a CFI Apochromat TIRF objective. Time-lapse images were acquired at 15 min frame intervals to minimize photobleaching and phototoxicity by high illumination and analyzed by 3D reconstruction of stacked axes. Imaging of 2D admixtures was performed using a fluorescence inverted microscope (Leica DMI3000 B). Cell tracking and fluorescence analyses were performed using the plug-in TrackMate in Fiji/lmageJ.

\section{Plasmids, RNAi, and transfections}

$60 \mathrm{nM}$ to $120 \mathrm{nM}$ target-specific smart pool (mix of at least two different sequences each; Supplemental Table 4) of short interfering RNAs (siRNAs) or non-targeting scrambled siRNA/siLuciferase (IDT Korea) were delivered with Lipofectamine 3000 (Life Technologies) or DharmaFECT (Dharmacon) according to manufacturer's instructions. Target siRNAs were obtained from IDT Korea, Life Technologies, or Bioneer. Unless otherwise specified, most assays were analyzed $48 \mathrm{~h}$ post-transfection. pCMV6-AC-Myc-DDK and pCMV6-FUT8-MycDDK (Origene) expression plasmids were delivered with Lipofectamine 3000 or FuGene 6 (Promega) according to manufacturer's instructions. Transfections were performed for $48 \mathrm{~h}$. To establish PON1 and SLC35C1 knockout (KO) cells, pLKO.1-puro or pLKO.1 plasmids encoding target shRNA constructs (Supplemental Table 4; selected from TRC shRNA Library, Broad; purchased from Origene) were cloned as previously described. The sequence of the constructs was verified by DNA sequencing (Origene). Scrambled shRNA (Addgene) was 
used as shControl. Lentiviral co-transfection of $8 \mu \mathrm{g}$ of cloned transgene plasmids, $1 \mu \mathrm{g}$ pMD2.G (envelope plasmid; Addgene), and $3 \mu \mathrm{g}$ psPAX2 (packaging plasmid; Addgene) was performed using iN-fect (Intron Biotechnology) in HEK293T cells following manufacturer's protocol and transduction in indicated cell lines using standard procedures. Lentivirus titer was determined using Lenti-X p24 rapid titer kit (Takara Bio). 2-8 $\mu \mathrm{g} / \mathrm{mL}$ puromycin was added gradually to select stable cell lines for two weeks. Stably selected KO cells were maintained in $0.1 \mu \mathrm{g} / \mathrm{mL}$ puromycin-containing complete media. To establish PON1-overexpressing cells, bicistronic pLVX-EF1 $\alpha$-IRES-puro (Takara Bio) encoding the CDS of human PON1 single mRNA transcript was cloned as previously described. The empty vector was used as a control (-CC). Co-transfection of plasmids, transduction, and selection were performed as above, except infected cells were selected in $1 \mu \mathrm{g} / \mathrm{mL}$ puromycin. Validation of targeted overexpression or RNAi is shown in Supplemental Fig. 25.

\section{Site-directed mutagenesis}

To generate mutant PON1 constructs, a PCR-based Q5 site-directed mutagenesis kit (NEB) was used according to the manufacturer's instructions. PON1 cDNA template was cloned into pcDNA3.1 (Genscript) as described previously ${ }^{[67]}$. The mutagenesis primers were designed using the Primer X Tool (http://bioinformatics.org/primerX/). FL or mutant PON1 constructs were transfected using Xfect transfection reagent (Clontech) according to manufacturer's protocol.

\section{Gene expression analysis}

Whole RNA (1-3 $\mu \mathrm{g}$ total per $10 \mu \mathrm{L}$ volume) was isolated using RNAeasy mini kit (QIAGEN) or TRIzol (Life Technologies) following manufacturer's protocol. Tumor tissues were homogenized in a handheld homogenizer using RLT-ME buffer (Qiagen). Complementary DNA (cDNA) was generated using the Transcriptor First Strand cDNA synthesis kit (Roche). RNA was treated with deoxyribonuclease I (DNase I; Takara) and reverse-transcribed using RevertAid reverse transcriptase (Fermentas). cDNA was amplified by an SYBR Green PCR master mix (Applied Biosystems). Differential RNA levels were assessed using Taqman gene expression assays (Life technologies). Quantitative polymerase chain reaction (qPCR) was performed using SureCycler 8800 (Agilent) and AriaMx Real-Time PCR System (Agilent). Relative gene expression was normalized to internal control genes: GAPDH or ACTB. For nucleic acids extraction (total RNA and genomic DNA) from FFPE tumor samples, we used FFPE All-Prep kit (QIAGEN) following the manufacturer's protocol. Small portion of specimens were prepared from $\sim 80-\mu \mathrm{m}$ slices of FFPE tumor blocks, followed by dewaxing using Deparaffinization Solution (QIAGEN). Purified RNA was subjected to reverse transcription PCR (RT-PCR) and qPCR as above. Primers used in this study are detailed in Supplemental Table 5.

\section{Immunoblotting and immunoprecipitation}

Enrichment of golgi and ER was carried out using Minute Golgi/ER enrichment kit (Invent Biotech) and isolation of nuclear and cytosolic extracts was carried out using NE-PER Nuclear and Cytoplasmic Extraction Reagents (Pierce) following the manufacturer's instructions. Whole-cell pellets were lysed as described previously ${ }^{[68]}$. Following surgery, xenograft tumors were flash frozen in liquid nitrogen. A portion of the frozen tumor excised from mice was thawed on ice and homogenized in Complete Lysis Buffer (Active Motif) for whole lysate extraction Protein concentrations were determined using Bradford reagent. Samples were boiled for $5 \mathrm{~min}$ in Laemmlli buffer. Equivalent amounts of proteins (usually 30-50 $\mu \mathrm{g}$ ) were separated by SDS-PAGE (usually on $7.5,10$, and $12 \%$ gels). For immunoprecipitation, PON1 monoclonal antibody was coupled to protein G-Sepharose 4B beads (GE Healthcare) and eluted as described previously[69]. Proteins were transferred onto Immobilon PVDF 
membranes (Millipore) using a semidry transfer system (Amersham). The detection system was Clarity Max Western ECL Substrate (Bio-Rad) and Western Lightning Plus-ECL (PerkinElmer). Secondary antibodies were either goat antibodies to mouse immunoglobulin G-horseradish peroxidase (IgG-HRP; DACO), mouse lgGk-HRP (Santa Cruz Biotechnology) or donkey antibodies to rabbit IgG-HRP (GE Healthcare). For cross-linking, cells were prestarved in media containing $2 \%$ dialyzed FBS prior to cross-linking using $1 \mathrm{mM}$ EGS for 45 min at $4^{\circ} \mathrm{C}$ as previously described. Briefly, lysates were diluted twofold in assay buffer and incubated with capture beads for FUT8 (protein G-agarose beads; Abcam) overnight. Lysates were clarified by centrifugation at $16,400 \mathrm{~g}$ for $15 \mathrm{~min}$, and then precleared for $30 \mathrm{~min}$ with agarose resin. Lysate was then incubated with protein A/G agarose and PON1 antibody overnight at $4^{\circ} \mathrm{C}$. The next day, the resin was washed six times with lysis buffer and then incubated with $2 \mathrm{M}$ hydroxylamine $\mathrm{HCl}$ in phosphate-buffered saline $(\mathrm{pH} 8.5)$ for $6 \mathrm{~h}$ at $37^{\circ} \mathrm{C}$. The resin was then removed, and the supernatant was used for indicated assay. Primary antibodies used for immunoblotting were PON1 (ab24261, Abcam), RCAS1 (12290, CST), and GAPDH (6C5, Santa Cruz Biotechnology). Antibodies used for immunoprecipitation were PON1 (17A12, Santa Cruz Biotechnology) and FUT8 (ab191571, Abcam).

\section{ELISA}

Sandwich-based ELISA kits were used to detect PON1 (RayBiotech), FUT8 (LSBio), and ATF6 (Novus Biologicals) following manufacturer's protocol. For ELISA detection of SLC35C1, 96well microtiter plates were manually pre-coated with SLC35C1 antibody (CSBPA839285LA01HU, Cusabio) similar to HLE. Absorbance was measured at $450 \mathrm{~nm}$.

\section{Polypeptide synthesis assay}

EZClick global protein synthesis kit (Biovision) was used to detect nascent protein synthesis following manufacturer's protocol. This assay is based on alkyne analog of puromycin, OPropargyl-puromycin (OP-puro). OP-puro stops translation by forming covalent conjugates with the nascent polypeptide chains. Truncated polypeptides are rapidly turned over by the proteasome and can be detected based on a click reaction with the fluorescent azide. Fluorescence was measured by flow cytometry using LSR Fortessa (BD Biosciences). Excitation and emission wavelengths were set at 440 and $530 \mathrm{~nm}$, respectively. Analysis was done using BD FACSDiva software.

\section{Trypsin sensitivity and $\mathrm{CHX}$ chase assays}

To evaluate the folding status of PON1, we exogenously treated lysates with trypsin as described previously ${ }^{[70]}$. Briefly, lysates were clarified by centrifugation at $17,800 \mathrm{~g}$ at $4^{\circ} \mathrm{C}$ for $10 \mathrm{~min} .1 \mathrm{mg} / \mathrm{mL} 50 \mu \mathrm{L}$ aliquots of cleared lysates were incubated with $2 \mu \mathrm{L}$ of indicated trypsin concentration (Promega) at $4^{\circ} \mathrm{C}$ for $15 \mathrm{~min} .50 \mu \mathrm{L}$ stop buffer ( $1 \times$ SDS sample buffer, $100 \mathrm{mM}$ dithiothreitol, 10x protease inhibitor cocktail) was added to the samples and incubated at $100^{\circ} \mathrm{C}$ for $5 \mathrm{~min} .30 \mathrm{\mu g}$ of each sample was separated by SDS-PAGE and immunoblotted. To evaluate PON1 stability, cultured cells in 6 -well plates were incubated with $25 \mu \mathrm{g} / \mathrm{mL} \mathrm{CHX}$ (Sigma) at indicated times. Cells were subjected to immunoblotting or other assays as indicated.

\section{Phospho-RTK array and kinase phosphorylation assays}

Phosphorylated RTKs were measured using PathScan human RTK signaling antibody array kit (R\&D Systems) according to manufacturer's instructions. Tyrosine 1068 phosphorylation of EGFR, pan-tyrosine phosphorylation of MET and HER3/ErbB3, and tyrosine 1150/1151 phosphorylation of Insulin Receptor $\beta$ were assessed by solid-phase sandwich ELISA (CST PathScan kits) following manufacturer's protocol. The assay quantitatively detects 
endogenous levels of the indicated targets. Absorbance was measured at $450 \mathrm{~nm}$.

\section{Cignal 45-pathway reporter array}

Cignal 45-pathway reporter arrays (QIAGEN) were used to simultaneously measure the activity of 45 transcription factors/signaling pathways according to manufacturer's protocol. Briefly, cell admixtures grown for 5 days under different conditions were transferred to Cignal Finder 96-well plates (at least 30,000 cells/well). Reporter constructs resident in each well were introduced into cells via reverse transfection. Cell admixtures were grown in Opti-MEM (Gibco) supplemented with 5\% FBS and $0.1 \mathrm{mM}$ MEM non-essential amino acids (NEAA; Gibco) for $48 \mathrm{~h}$. Cell admixtures were then lysed and luciferase activity was measured using dual-emission optics of a plate reader (POLARstar Omega).

\section{Proliferation, survival, cell cycle, apoptosis, and senescence assays}

Cell proliferation and survival were assessed by sulforhodamine $B$ (SRB) and colony formation assays, as we previously described ${ }^{[69]}$. Cell sorting, cell cycle analysis by quantitation of DNA content, and cell death detection in the sub-G1 peak were performed by flow cytometry as we previously described using FACSCalibur (BD Biosciences) ${ }^{[69]}$. Analysis was done using BD CellQuest Pro software. At least 20,000 cells were used for each analysis. Changes in the percentage of cell distribution at each phase of the cell cycle were determined. To isolate apoptotic bodies, cells grown in indicated conditions were transferred to serum-free media with $0.35 \%$ BSA and cell debris was collected after $24 \mathrm{~h}$. Cells were centrifuged at $300 \mathrm{~g}$ for $10 \mathrm{~min}$, the remaining cell debris was removed, and the soluble secretome was collected. The mixture was centrifuged at $16,500 \mathrm{~g}$ for 20 min using a super speed vacuum centrifuge (Vision Scientific). To detect senescence, we measured SA- $\beta$-gal activity using senescence $\beta$ galactosidase staining kit (CST) following manufacturer's protocol. SA- $\beta$-gal positive cells were quantified based on three independent images from different stained regions analyzed by digital inverted light microscopy (40× phase-contrast; Leica DMi1). To evaluate senescent gene signature and SASP activity, we measured the gene expression of $\mathrm{p} 16$, LNMB1, IL-1 $\alpha$, IL-6, MMP-3, MMP-9, CXCL-1, CXCL-10, and CCL20 by qPCR.

\section{Caspase activity and intracellular ATP assays}

Caspase $3 / 7$ and 9 activities were assessed using a fluorescence-based Apo-ONE homogenous caspase 3/7 assay kit (Promega) and luminescence-based caspase-glo 9 assay system (Promega), respectively, following manufacturer's protocol. Excitation and emission wavelengths were set at 560 and $590 \mathrm{~nm}$, respectively. Luminescence was read on luminometer. For ATP measurement, cells were seeded in 96-well plates and were subjected to indicated treatment/culture conditions all in nutrient-restricted media (10\% dialyzed FBS). ATP levels were measured using the luminescence-based ATPLite system (Perkin-Elmer) following manufacturer's instructions.

\section{Enzyme activity assays}

Paraoxonase activity was assessed based on 4-nitrophenol formation as described previously ${ }^{[71]}$. Paraoxon (O,O-Diethyl O-(4-nitrophenyl) phosphate; Sigma) was used as a substrate. Absorbance was measured at $412 \mathrm{~nm}$. One unit of paraoxonase activity was defined as $1 \mathrm{nM}$ of 4-nitrophenol formed per min. Arylesterase activity was assessed based on phenol formation as described previously ${ }^{[71]}$. Phenylacetate (Sigma) was used as a substrate. Absorbance was measured at $217 \mathrm{~nm}$. One unit of arylesterase activity is equal to $1 \mathrm{mM}$ of phenylacetate hydrolyzed per min. 


\section{ROS/RNS and cytokine measurements}

Free radical ROS/RNS was measured using OxiSelect in vitro ROS/RNS assay kit (Cell Biolabs) according to manufacturer's protocol. This assay used DCFH probe and oxidative reactions were measured against $\mathrm{H} 2 \mathrm{O} 2$ or DCF standard. Excitation and emission wavelengths were set at 480 and $530 \mathrm{~nm}$, respectively. IL-6, TFN- $\alpha$, and GM-CSF levels were quantified using ELISA kits pre-coated with indicated capture antibodies per manufacturer's instructions (Sigma). IL-6 levels were preliminarily detected using a Q-Plex Human cytokine screen (16-plex; Quansys Biosciences). Absorbance was measured at $450 \mathrm{~nm}$.

\section{Immunofluorescence}

Cells were plated onto $0.1 \%$ gelatin-coated glass-bottom 30 -mm dishes (except for 3D tumor spheroids) and incubated overnight unless otherwise specified. The cells were fixed with $4 \%$ paraformaldehyde (in PBS) for $8 \mathrm{~min}$ at RT, quenched for $1 \mathrm{~min}$ in $10 \mathrm{mM}$ Tris (in PBS) at RT, and permeabilized in $0.1 \%$ Triton X-100 (in PBS). Cells were then blocked in $2 \%$ bovine serum albumin (BSA) (in PBS containing $0.01 \%$ Triton X-100) for 30 min at RT and incubated with primary antibodies diluted in $2 \% \mathrm{BSA}$ for $2 \mathrm{~h}$. Alexa Fluor or fluorescein isothiocyanateconjugated secondary antibodies were used to label primary antibodies. DAPI (4',6-diamidino2-phenylindole; $0.35 \mu \mathrm{g} / \mathrm{ml}$ ) was used to counterstain the nuclei. Cells were mounted using VECTASHIELD Mounting Medium (Vector Laboratories). Confocal microscopy was carried out using a ZEISS LSM 780 ApoTome microscope (Carl Zeiss) using C-Apochromat 40× lens with a numerical aperture of 1.20. Primary antibodies used for immunofluorescence were PON1 (ab24261, Abcam), PON3 (ab42322, Abcam), ATF6 (PA5-20215, Invitrogen), RCAS1 (12290, CST), and XBP1 (ab37152, Abcam).

\section{Immunohistochemistry}

Human or mouse FFPE tumor tissue sections were deparaffinized in xylene alternative (HistoClear, EMS; $3 \times 5 \mathrm{~min})$ and rehydrated in $\mathrm{EtOH} / \mathrm{H} 2 \mathrm{O}$ gradient series $(100 \%, 95 \%, 70 \%, 40 \%$, $5 \mathrm{~min}$ each). The rehydrated sections were washed in TBS for $10 \mathrm{~min}$. Epitopes were unmasked using heat-induced retrieval method with the use of the pre-heated Tris $(10 \mathrm{mM}$ Tris base, $0.05 \%$ Tween $20, \mathrm{pH} 8.0$ ) and citrate buffers ( $10 \mathrm{mM}$ sodium citrate, $0.05 \%$ Tween 20, $\mathrm{pH}$ 6.0). Sections were pressure-cooked for $12 \mathrm{~min}$ in Tris buffer, transferred to citrate buffer, heated for $12 \mathrm{~min}$, cooled at RT for $40 \mathrm{~min}$, and washed with TBS containing $0.1 \%$ Twee-20 for 10 min. Sections were permeabilized with $0.3 \%$ Triton X-100 in TBS for 45 min and washed in TBS $(2 \times 5 \mathrm{~min})$. Endogenous peroxidase activity was quenched in a peroxidase solution $(0.3 \% \mathrm{H} 2 \mathrm{O} 2$ in TBS) and sections were blocked in a universal blocking solution $(10 \%$ normal donkey serum in 1\% BSA/TBS) or carbo-free blocking solution (for fucosylation detection) for $2 \mathrm{~h}$. Slides were blotted to remove the serum and then primary antibodies were applied at predetermined concentrations (1:400 or 1:800). Slides were incubated overnight at $4^{\circ} \mathrm{C}$ in a humidified chamber and washed with TBS $(3 \times 5 \mathrm{~min})$. Biotinylated link and HRPconjugated secondary antibodies were applied onto sections and were incubated for $2 \mathrm{~h}$ in a dark humidified chamber RT followed by washing. Replicate slides were also stained H\&E (Vector Labs) according to manufacturer's protocol. A VECTASHIELD hard set mounting medium (Vector Labs) was used to mount the slides. The positive staining density was measure using a Leica CCD camera connected to a Leica DMi1 microscope. Biotinylated AAL $(20 \mu \mathrm{g} / \mathrm{ml})$ was used to detect fucosylation. Primary antibodies used were PON1 (18155-1-AP, Protein tech) and PON3 (OTI1A5, Thermo Scientific).

\section{Xenografts and induction of lung metastases}

Procedures were performed at the College of Veterinary Medicine, Seoul National University following guidelines approved by the Institutional Animal Care and Use Committee. 5-7 week 
old C57BL/6 background male mice were purchased from Orient Bio Inc. and fed with free access to standard diet (PMI LabDiet) and water. For xenografts, LLC-CC and LLC-PON1 cell suspensions $\left(1.2 \times 10^{7}\right.$ cells $)$ in $200 \mu \mathrm{L}$ of culture medium/growth factor-reduced Matrigel (BD Biosciences) in a 1:1 ratio were subcutaneously injected into the right flank of each mouse. For metastases, LLC-CC and LLC-PON1 cell suspensions $\left(2.5 \times 10^{6}\right.$ cells $)$ in $150 \mu \mathrm{L}$ culture media were intravenously injected through the tail vein. Mice were sacrificed at indicated times after injection (Supplemental Fig. 24, A and M). Lung, liver, spleen, and other sites that developed primary or metastasized tumors were collected in ice-cold PBS for further testing. All mice were maintained under continuous sedation by administering $2-4 \%$ isoflurane (Sigma) via an anesthesia mask during surgery and prior to euthanasia. Tumor volume was determined using digital caliper measurements and calculated using the following formula: tumor volume $=(D \times d 2) / 2$, in which $D$ and $d$ refer to the long and short tumor diameter, respectively. The body weight of each mouse was also monitored. To sample serum, mice were isolated in a cage under an infrared lamp for few min to increase blood flow. Portion of the tails were swabbed and blood was collected. Blood samples were transferred to tubes, incubated at $4^{\circ} \mathrm{C}$ for at least $4 \mathrm{~h}$, and centrifuged at $10,000 \mathrm{~g}$ at $4^{\circ} \mathrm{C}$ for $10 \mathrm{~min}$. Serum was collected and recentrifuged and "snap frozen" in liquid nitrogen until further use.

\section{Transwell invasion and gap-closure assays}

Chemotactic invasion assay was carried out in Boyden chamber wells (24-well format, $8 \mu \mathrm{m}$ pore size; Corning) with matrigel/ECM-based membrane. Matrigel matrix (Corning) was diluted to $1 \mathrm{mg} / \mathrm{mL}$ with serum-free Opti-MEM and applied on the insert in the upper chambers. For chemotaxis induction of cells, $800 \mu \mathrm{L}$ culture media supplemented with $8 \%$ FBS or indicated mouse serum was added to the lower chambers. After incubation for $24 \mathrm{~h}$ or $48 \mathrm{~h}$ at standard culture conditions, the membrane inserts were removed and the non-invading cells were removed from the upper surface of the membrane while invading cells were removed non-enzymatically using $1 \times$ cell dissociation solution (Corning) following manufacturer's protocol. Invaded cells were stained with Mayer's modified hematoxylin (Abcam) for 20 min and washed with water. For gap closing assay, cells were seeded and grown until confluent. A P10 tip was used to generate a gap. Cells were washed and secretomes were added. Images were acquired overtime to monitor gap closure using a digital inverted light microscopy.

\section{Label-free proteomics}

Precipitated AAL-enriched secretomes $(45 \mu \mathrm{g})$ were run on a $1 \mathrm{~mm}$ thick $10 \%$ SDS-PAGE gel and stained with CBB G-250 staining solution (Bio-Rad) at RT for 1.5 h. 30-70 kDa lane portions were excised into $2 \times 2 \mathrm{~mm}$ cubes and transferred to Protein Lo-Bind tubes (Eppendorf). Excised gels were partitioned into tubes, and destained multiple times in $75 \mathrm{mM}$ ammonium bicarbonate (Sigma) and $40 \% \mathrm{EtOH}(1: 1)$ in a shaking rack. Destained gel pieces were washed with $100 \mathrm{mM}$ ammonium bicarbonate and acetonitrile (1:1), vortexed, and incubated at RT for $15 \mathrm{~min}$. Gel pieces were diluted with $100 \mathrm{mM}$ ammonium bicarbonate and reduced with $10 \mathrm{mM}$ dithiothreitol at $51^{\circ} \mathrm{C}$ for $1 \mathrm{~h}$. Gel pieces were cooled down to RT for 30 min followed by alkylation with $20 \mathrm{mM}$ of iodoacetamide at RT for $45 \mathrm{~min}$ in the dark. Gel pieces were dehydrated in $100 \%$ acetonitrile and dried in a SpeedVac. In-gel proteins were digested with trypsin at a protein:enzyme ratio of $20: 1$ at $37^{\circ} \mathrm{C}$ for $12 \mathrm{~h}$ in a shaking incubator. Peptides were extracted in $100 \mu \mathrm{L}$ extraction buffer $(5 \%$ acetic acid/acetonitrile; $1: 2)$ and incubated at $37^{\circ} \mathrm{C}$ for $15 \mathrm{~min}$ in a shaking incubator. Tryptic peptide mixture was eluted from the gel with $0.1 \%$ acetic acid.

Mass spectrometry was performed as we described previously ${ }^{[49]}$. Briefly, nanospray liquid chromatography tandem mass spectrometry (LC-MS/MS) was performed on an LTQ-Orbitrap mass spectrometer (Thermo Electron) coupled to Agilent 1200 series G1312B binary pump SL and NanoLC AS-2 autosampler (Eksigent Technologies). Peptide mixtures (2 $\mu \mathrm{L}$ per 
sample) were loaded via the autosampler on $75-\mu \mathrm{m}$ (inner diameter) fused silica capillary columns with electrospray tip packed with C18 reversed phase resin (Magic C18, 5- $\mu \mathrm{m}$ particles, 200-Å pore size; Michrom BioResources). Peptides were separated by reversed phase liquid chromatography with mobile phases as we described previously. The tandem mass spectra were processed using Sorcerer 3.4 beta2 (Sorcerer Web interface). All MS/MS samples were analyzed using SEQUEST Cluster (Thermo Scientific) and Mascot generic format (MGF) files were set to query the human IPI v3.68 database. Searches were performed with and without oxidation of methionine and carbamidomethyl modification of cysteine as variable modifications. False positives and false discovery rates were calibrated through the decoy option during data search in Sorcerer to reduce noise effects. Scaffold v4.0.5 (Proteome Software) was used to validate MS/MS-based peptide and protein identification. PeptideProphet was used to validate peptide and protein assignments to MS/MS spectra (>95\% probability). Subtractive proteomic analysis for each dataset was performed by normalization using total ion current (TIC; normalized by average of all the TIC values of the spectra assigned to a protein). MS .RAW files were processed in MaxQuant ${ }^{[72]}$, version 1.5.5.2. The FASTA file Homo_sapiens.GRCh38.pep.all.fa was downloaded from Ensembl.

\section{RNA-seq}

Low density H1993-GR were grown under indicated conditions for $48 \mathrm{~h}$ (Fig. 6A) and whole RNA was extracted using the RNAeasy mini kit. $2 \times 101$ paired-end RNA-seq libraries were constructed using TruSeq stranded total RNA H/M/R prep kit and sequenced using the Novaseq6000 system (Illumina). Raw paired-end sequencing reads were mapped to the human genome (build hg38) with HISAT2 v2.1.0 using default parameters except with the options "--dta" and "--dta-cufflinks". Stringtie v.2.0.6 was used to quantify the expression of genes and transcripts by employing transcriptome information from GENCODE v27. Ballgown package was used to perform differential gene expression analysis generating FPKM for each gene. Genes with FDR $<0.05$, fold change larger than 2 or smaller than 0.7 -fold, and average read counts larger than 10 were treated as differentially expressed genes. Gene ontology analysis was performed using DAVID 6.8. GO of biological process or molecular function were detected and summarized. GO terms with $\mathrm{P}<0.01$ were selected as significant. Semanticsimilarity network visualization of GO terms were done using REVIGO (www.revigo.irb.hr). Hierarchical clustering was performed using pheatmap library in R. Row-value filtered FPKM values were analyzed using default options. Heatmap colors indicate z-score in each row (Supplemental Fig. 22A).

\section{Bioinformatics}

Drug response (as IC50 per drug and cell line) and gene expression data (as log2 transformed RMA normalized basal expression or RNA-seq TPM expression per cell line) were derived from GDSC (v2, accessed from www.cancerRxgene.org) and CCLE (v2, accessed from www.depmap.org) projects. All IC50s are expressed in $\mu \mathrm{M}$. Categorical grouping of cell lines per cancer type was done and plotted in $\mathrm{R}$ (see code availability). Discretization threshold for each drug (log IC50/cell line) was determined as described previously ${ }^{[30,68,69]}$. Cell lines without corresponding drug or expression measurement were not included in the analysis. Drug sensitivity data were evaluated using IC50 values (including extrapolated values) for GDSC and activity area for CCLE. Correlation analysis between drug response and gene expression per cancer type were performed by quantitatively matching pre-processed values. All Spearman's correlation coefficients, relative quantitation, and plotting were performed in Python (see code availability). Only correlations with $p<0.05$ are shown. Summary reference on drug categories and target pathways is accessible in GDSC (available as Excel file TableS1F.xlsx in their database). Mutation dataset were obtained from CCLE (v2, accessed from www.cbioportal.org) and FUT domain information were searched in the Pfam database (www.pfam.xfam.org). CTRP dataset was analyzed using the CARE algorithm 
(www.care.dfci.harvard.edu).

CCS gene set $(n=1,810)$ was obtained and filtered from UniProt as described previously ${ }^{[8]}$. Glycosylation (N-/O-glycosylation) gene sets were obtained by conducting gene set analysis using the GOs "glycosylation", "protein N-linked glycosylation", and "protein O-linked glycosylation" from MSigDB. The GO "glycosylation" contains 22 annotated sub-GOs. These gene sets were used in their complete form, and were not filtered. Both total and overlapping glycosylation genes $(n=264$ or 19 for overall glycosylation, $n=81$ or 1 for $N$-linked, and $n=193$ or 18 for O-linked) with the CCS gene set were included in the analysis. For CCS, missing values on FC_2 were removed and 0 was considered as missing value. $P$ values were calculated by two-sided Student's $t$-test and adjusted ( $p_{\text {adj }}$ ) to control for FDR using BenjaminiHochberg procedure. Methylation data analysis was performed using the pre-processed reduced representation bisulfite sequencing (RRBS) dataset from CCLE v2. Drug sensitivity data were obtained from GDSC as mentioned above.

PON1 co-expressing genes were obtained from CCLE v2 based on RNA-seq RPKM mRNA abundance data. Interaction rank was based on Spearman's correlation and $p$ value. Quantitative analysis and plotting were done in Python (see code availability). Localization for each gene-encoding protein were queried in the Human Protein Atlas (www.proteinatlas.org).

$\mathrm{N}$-glycosylation sites from the PON1 protein sequence were predicted using NetNGlyc (www.cbs.dtu.dk/services/NetNGlyc). Folded and charged regions within PON1 were visualized with Foldlndex (www.fold.weizmann.ac.il/fldbin/findex) and EMBOSS charge prediction tool (www.bioinformatics.nl/cgi-bin/emboss/charge). Functional protein stability and folding effects of specific amino acid substitutions were predicted using MutPred v2 (www.mutpred.mutdb.org) and I-Mutant v3 (www.gpcr2.biocomp.unibo.it/cgi/predictors/lMutant3.0/l-Mutant3.0.cgi).

Cancer dependency profiles were obtained from the DepMap portal (www.depmap.org/portal) RNAi screen dataset (CRISPR Avana Public 20Q2). Dependency scores across all cancer types were grouped by lineage type as predefined by DepMap and were subsequently used for correlation analysis with drug response for indicated targeted therapy obtained from GDSC v2 (AUC values). Spearman's correlation coefficients and linear regression-derived $p$ values were obtained from pre-computed associations in the DepMap portal. Lineages with less than four cell lines for a specific gene inquiry were removed from the dataset. Raw essentiality scores were derived from the Profiling Relative Inhibition Simultaneously in Mixtures (PRISM) drug screen and Project Achilles gene dependency screen both from the Broad Institute.

For patient survival analysis (first progression or RFS), the data were queried in the KM plotter (www.kmplot.com/analysis) for lung cancer or pan-cancer. For co-occurrence gene analysis, data from the breast cancer METABRIC cohort were used (accessed from the cBioPortal). The co-occurring genes in patients with indicated fucosylation gene copy number amplification, deep deletion, mRNA upregulation, or mRNA downregulation were stratified.

\section{References}

1. D. S. Krause, R. A. van Etten, Tyrosine kinases as targets for cancer therapy. N. Engl. J. Med. 353, 172-187 (2005).

2. J. Baselga, Targeting tyrosine kinases in cancer: the second wave. Science $312,1175-1178$ (2006).

3. J. J. Lin, A. T. Shaw, Resisting resistance: targeted therapies in lung cancer. Trends Cancer 2, 350-364 (2016). 
4. P. B. Chapman, A. Hauschild, C. Robert, J. B. Haanen, P. Ascierto, J. Larkin, R. Dummer, C. Garbe, A. Testori, M. Maio, D. Hogg, P. Lorigan, C. Lebbe, T. Jouary, D. Schadendorf, A. Ribas, S. J. O'Day, J. A. Sosman, J. M. Kirkwood, A. M. M. Eggermont, B. Dreno, K. Nolop, J. Li, B. Nelson, J. Hou, R. J. Lee, K. T. Flaherty, G. A. McArthur, BRIM-3 Study Group, Improved survival with vemurafenib in melanoma with BRAF V600E mutation. N. Engl. J. Med. 364, 2507-2516 (2011).

5. C. L. Arteaga, M. X. Sliwkowski, C. K. Osborne, E. A. Perez, F. Puglisi, L. Gianni, Treatment of HER2-positive breast cancer: current status and future perspectives. Nat. Rev. Clin. Oncol. 9, 16-32 (2011).

6. R. Salgia, P. Kulkarni, The genetic/non-genetic duality of drug 'resistance' in cancer. Trends Cancer 4, 110-118 (2018).

7. A. C. Obenauf, Y. Zou, A. L. Ji, S. Vanharanta, W. Shu, H. Shi, X. Kong, M. C. Bosenberg, T. Wiesner, N. Rosen, R. S. Lo, J. Massagué, Therapy-induced tumour secretomes promote resistance and tumour progression. Nature 520, 368-372 (2015).

8. J. L. Robinson, A. Feizi, M. Uhlén, J. Nielsen, A systematic investigation of the malignant functions and diagnostic potential of the cancer secretome. Cell Rep. 26, 2622-2635 (2019).

9. S. M. Hanash, S. J. Pitteri, V. M. Faca, Mining the plasma proteome for cancer biomarkers. Nature 452, 571-579 (2008).

10. R. Kalluri, V. S. LeBleu, The biology, function, and biomedical applications of exosomes. Science 367, eaau6977 (2020).

11. G. P. Gupta, J. Massagué, Cancer metastasis: building a framework. Cell 127, 679-695 (2006).

12. M. Uhlén, L. Fagerberg, B. M. Hallström, C. Lindskog, P. Oksvold, A. Mardinoglu, Å. Sivertsson, C. Kampf, E. Sjöstedt, A. Asplund, I. Olsson, K. Edlund, E. Lundberg, S. Navani, C. A. Szigyarto, J. Odeberg, D. Djureinovic, J. O. Takanen, S. Hober, T. Alm, P. Edqvist, H. Berling, H. Tegel, J. Mulder, J. Rockberg, P. Nilsson, J. M. Schwenk, M. Hamsten, K. von Feilitzen, M. Forsberg, L. Persson, F. Johansson, M. Zwahlen, G. von Heijne, J. Nielsen, F. Pontén, Tissue-based map of the human proteome. Science 347, 1260419 (2015).

13. C. K. Barlowe, E. A. Miller, Secretory protein biogenesis and traffic in the early secretory pathway. Genetics 193, 383-410 (2013).

14. E. C. Madden, A. M. Gorman, S. E. Logue, A. Samali, Tumour cell secretome in chemoresistance and tumour recurrence. Trends Cancer 6, 489-505 (2020).

15. J. M. Pitt, A. Marabelle, A. Eggermont, J. Soria, G. Kroemer, L. Zitvogel, Targeting the tumor microenvironment: removing obstruction to anticancer immune responses and immunotherapy. Ann. Oncol. 27, 1482-1492 (2016).

16. R. G. Spiro, Protein glycosylation: nature, distribution, enzymatic formation, and disease implications of glycopeptide bonds. Glycobiology 12, 43-56 (2002).

17. C. Reily, T. J. Stewart, M. B. Renfrow, J. Novak, Glycosylation in health and disease. Nat. Rev. Nephrol. 15, 346-366 (2019).

18. X. Zhang, Y. Wang, Glycosylation quality control by the golgi structure. J. Mol. Biol. 428, 3183-3193 (2016).

19. D. Wlodkowic, J. Skommer, D. McGuinness, C. Hillier, Z. Darzynkiewicz, ER-Golgi network-a future target for anti-cancer therapy. Leuk. Res. 33, 1440-1447 (2009).

20. R. G. Lichtenstein, G. A. Rabinovich, Glycobiology of cell death: when glycans and lectins govern cell fate. Cell Death Differ. 20, 976-986 (2013).

21. J. R. Cubillos-Ruiz, S. E. Bettigole, L. H. Glimcher, Tumorigenic and immunosuppressive effects of endoplasmic reticulum stress in cancer. Cell 168, 692-706 (2017).

22. A. F. Costa, D. Campos, C. A. Reis, C. Gomes, Targeting glycosylation: a new road for cancer drug discovery. Trends Cancer S2405-8033, 30129-1 (2020).

23. P. Pothukuchi, I. Agliarulo, D. Russo, R. Rizzo, F. Russo, S. Parashuraman, Translation of genome to glycome: role of the Golgi apparatus. FEBS Lett. 593, 2390-2411 (2019). 
24. S. S. Pinho, C. A. Reis, Glycosylation in cancer: mechanisms and clinical implications. Nat. Rev. Cancer 15, 540-555 (2015).

25. G. Lauc, M. Pezer, I. Rudan, H. Campbell, Mechanisms of disease: The human N-glycome. Biochim. Biophys. Acta 1860, 1574-1582 (2016).

26. O. M. T. Pearce, Cancer glycan epitopes: biosynthesis, structure and function. Glycobiology 28, 670-696 (2018).

27. A. Blanas, N. M. Sahasrabudhe, E. Rodríguez, Y. van Kooyk, S. J. van Vliet, Fucosylated antigens in cancer: an alliance toward tumor progression, metastasis, and resistance to chemotherapy. Front. Oncol. 8, 39 (2018).

28. M. Schneider, E. Al-Shareffi, R. S. Haltiwanger, Biological functions of fucose in mammals. Glycobiology 27, 601-618 (2017).

29. T. S. Keeley, S. Yang, E. Lau, The diverse contributions of fucose linkages in cancer. Cancers 11, 1241 (2019).

30. F. Iorio, T. A. Knijnenburg, D. J. Vis, G. R. Bignell, M. P. Menden, M. Schubert, N. Aben, E. Gonçalves, S. Barthorpe, H. Lightfoot, T. Cokelaer, P. Greninger, E. van Dyk, H. Chang, H. de Silva, H. Heyn, X. Deng, R. K. Egan, Q. Liu, T. Mironenko, X. Mitropoulos, L. Richardson, J. Wang, T. Zhang, S. Moran, S. Sayols, M. Soleimani, D. Tamborero, N. Lopez-Bigas, P. RossMacdonald, M. Esteller, N. S. Gray, D. A. Haber, M. R. Stratton, C. H. Benes, L. F. A. Wessels, J. Saez-Rodriguez, U. McDermott, M. J. Garnett, A landscape of pharmacogenomic interactions in cancer. Cell 166, 740-754 (2016).

31. J. Barretina, G. Caponigro, N. Stransky, K. Venkatesan, A. A. Margolin, S. Kim, C. J. Wilson, J. Lehár, G. V. Kryukov, D. Sonkin, A. Reddy, M. Liu, L. Murray, M. F. Berger, J. E. Monahan, P. Morais, J. Meltzer, A. Korejwa, J. Jané-Valbuena, F. A. Mapa, J. Thibault, E. Bric-Furlong, P. Raman, A. Shipway, I. H. Engels, J. Cheng, G. K. Yu, J. Yu, P. Aspesi Jr, M. de Silva, K. Jagtap, M. D. Jones, L. Wang, C. Hatton, E. Palescandolo, S. Gupta, S. Mahan, C. Sougnez, R. C. Onofrio, T. Liefeld, L. MacConaill, W. Winckler, M. Reich, N. Li, J. P. Mesirov, S. B. Gabriel, G. Getz, K. Ardlie, V. Chan, V. E. Myer, B. L. Weber, J. Porter, M. Warmuth, P. Finan, J. L. Harris, M. Meyerson, T. R. Golub, M. P. Morrissey, W. R. Sellers, R. Schlegel, L. A. Garraway, The Cancer Cell Line Encyclopedia enables predictive modelling of anticancer drug sensitivity. Nature 483, 603-607 (2012).

32. Cancer Cell Line Encyclopedia Consortium; Genomics of Drug Sensitivity in Cancer Consortium, Pharmacogenomic agreement between two cancer cell line data sets. Nature 528, 84-87 (2015).

33. P. M. Haverty, E. Lin , J. Tan, Y. Yu , B. Lam, S. Lianoglou, R. M. Neve, S. Martin, J. Settleman, R. L .Yauch, R. Bourgon, Reproducible pharmacogenomic profiling of cancer cell line panels. Nature 533, 333-337 (2016).

34. Q. Yang, R. Zhang, H. Cai, L. Wang, Revisiting the substrate specificity of mammalian a1,6fucosyltransferase reveals that it catalyzes core fucosylation of N-glycans lacking a1,3-arm GlcNAc. J. Biol. Chem. 292, 14796-14803 (2017).

35. P. Jiang, W. Lee, X. Li, C. Johnson, J. S. Liu, M. Brown, J. C. Aster, X. S. Liu, Genome-scale signatures of gene interaction from compound screens predict clinical efficacy of targeted cancer therapies. Cell Syst. 6, 343-354 (2018).

36. R. Saldova, E. Dempsey, M. Pérez-Garay, K. Mariño, J. A. Watson, A. Blanco-Fernández, W. B. Struwe, D. J. Harvey, S. F. Madden, R. Peracaula, A. McCann, P. M. Rudd, 5-AZA-2'deoxycytidine induced demethylation influences $\mathrm{N}$-glycosylation of secreted glycoproteins in ovarian cancer. Epigenetics 6, 1362-1372 (2011).

37. A. Wahl, S. Kasela, E. Carnero-Montoro, M. van Iterson, J. Štambuk, S. Sharma, E. van den Akker, L. Klaric, E. Benedetti, G. Razdorov, I. Trbojević-Akmačić, F. Vučković, I. Ugrina, Marian Beekman, J. Deelen, D. van Heemst, B. T. Heijmans, BIOS Consortium, M. Wuhrer, R. Plomp, T. Keser, M. Šimurina, T. Pavić, I. Gudelj, J. Krištić, H. Grallert, S. Kunze, A. Peters, J. T. Bell, T. D. Spector, L. Milani, P. E. Slagboom, G. Lauc, C. Gieger, IgG glycosylation and 
DNA methylation are interconnected with smoking. Biochim. Biophys. Acta 1862, 637-648 (2018).

38. P. A. Jones, Functions of DNA methylation: islands, start sites, gene bodies and beyond. Nat. Rev. Genet. 13, 484-492 (2012).

39. T. Wang, S. Zheng, L. Liu, J. Voglmeir, Development of a colorimetric PNGase activity assay. Carbohydr. Res. 472, 58-64 (2019).

40. H. H. Freeze, C. Kranz, Endoglycosidase and glycoamidase release of N-linked glycans. Curr. Protoc. Protein Sci. 12, 12.4 (2010).

41. R. B. Trimble, A. L. Tarentino, Identification of distinct endoglycosidase (endo) activities in Flavobacterium meningosepticum: endo F1, endo F2, and endo F3. Endo $F 1$ and endo $H$ hydrolyze only high mannose and hybrid glycans. J. Biol. Chem. 266, 1646-1651 (1991).

42. M. Collin, A. Olsén, EndoS, a novel secreted protein from Streptococcus pyogenes with endoglycosidase activity on human IgG. EMBO J. 20, 3046-3055 (2001).

43. J. J. Goodfellow, K. Baruah, K. Yamamoto, C. Bonomelli, B. Krishna, D. J. Harvey, M. Crispin, C. N. Scanlan, B. G. Davis, An endoglycosidase with alternative glycan specificity allows broadened glycoprotein remodelling. J. Am. Chem. Soc. 134, 8030-8033 (2012).

44. A. Tsherniak, F. Vazquez, P. G. Montgomery, B. A. Weir, G. Kryukov, G. S. Cowley, S. Gill, W. F. Harrington, S. Pantel, J. M. Krill-Burger, R. M. Meyers, L. Ali, A. Goodale, Y. Lee, G. Jiang, J. Hsiao, W. F. J. Gerath, S. Howell, E. Merkel, M. Ghandi, L. A. Garraway, D. E. Root, T. R. Golub, J. S. Boehm, W. C. Hahn, Defining a cancer dependency map. Cell 170, 564-576 (2017).

45. C. V. Pecot, S. Y. Wu, S. Bellister, J. Filant, R. Rupaimoole, T. Hisamatsu, R. Bhattacharya, A. Maharaj, S. Azam, C. Rodriguez-Aguayo, A. S. Nagaraja, M. P. Morelli, K. M. Gharpure, T. A. Waugh, V. Gonzalez-Villasana, B. Zand, H. J. Dalton, S. Kopetz, G. Lopez-Berestein, L. M. Ellis, A. K. Sood, Therapeutic silencing of KRAS using systemically delivered siRNAs. Mol. Cancer Ther. 13, 2876-2885 (2014).

46. K. Xu, D. Park, A. T. Magis, J. Zhang, W. Zhou, G. L. Sica, S. S. Ramalingam, W. J. Curran, X. Deng, Small molecule KRAS agonist for mutant KRAS cancer therapy. Mol. Cancer 19, 93 (2020).

47. J. Massagué, A. C. Obenauf, Metastatic colonization by circulating tumour cells. Nature 529 , 298-306 (2016).

48. M. LaMantia, T. Miura, H. Tachikawa, H. A. Kaplan, W. J. Lennarz, T. Mizunaga, Glycosylation site binding protein and protein disulfide isomerase are identical and essential for cell viability in yeast. Proc. Natl. Acad. Sci. USA 88, 4453-4457 (1991).

49. J. Ahn, H. Sung, Y. Yoon, B. Kim, W. S. Yang, C. Lee, H. Park, B. Kim, B. Kim, S. Lee, H. An, J. Cho, Integrated glycoproteomics demonstrates fucosylated serum paraoxonase 1 alterations in small cell lung cancer. Mol. Cell. Proteomics 13, 30-48 (2014).

50. M. B. D. Aldonza, Y. S. Son, H. Sung, J. M. Ahn, Y. Choi, Y. Kim, S. Cho, J. Cho, Paraoxonase-1 (PON1) induces metastatic potential and apoptosis escape via its antioxidative function in lung cancer cells. Oncotarget 8, 42817-42835 (2017).

51. M. Harel, A. Aharoni, L. Gaidukov, B. Brumshtein, O. Khersonsky, R. Meged, H. Dvir, R. B. G. Ravelli, A. McCarthy, L. Toker, I. Silman, J. L. Sussman, D. S. Tawfik, Structure and evolution of the serum paraoxonase family of detoxifying and anti-atherosclerotic enzymes. Nat. Struct. Mol. Biol. 11, 412-419 (2004).

52. D. I. Draganov, J. F. Teiber, A. Speelman, Y. Osawa, R. Sunahara, B. N. L. Du, Human paraoxonases (PON1, PON2, and PON3) are lactonases with overlapping and distinct substrate specificities. J. Lipid Res. 46, 1239-1247 (2005).

53. M. R. M. Hussain, Z. Iqbal, W. M. Qazi, D. C. Hoessli, Charge and polarity preferences for Nglycosylation: a genome-wide in silico study and its implications regarding constitutive proliferation and adhesion of carcinoma cells. Front. Oncol. 8, 29 (2018).

54. S. H. Shakin-Eshleman, S. L. Spitalnik, L. Kasturi, The amino acid at the $X$ position of an Asn$\mathrm{X}$-Ser sequon is an important determinant of $\mathrm{N}$-linked core-glycosylation efficiency. J. Biol. 
Chem. 271, 6363-6366 (1996).

55. A. García-García, L. Ceballos-Laita, S. Serna, R. Artschwager, N. C. Reichardt, F. Corzana, R. Hurtado-Guerrero, Structural basis for substrate specificity and catalysis of a1,6fucosyltransferase. Nat. Commun. 11, 973 (2020).

56. T. H. Tseng, T. W. Lin, C. Y. Chen, C. H. Chen, J. L. Lin, T. L. Hsu, C. H. Wong, Substrate preference and interplay of fucosyltransferase 8 and $\mathrm{N}$-acetylglucosaminyltransferases. J. Am. Chem. Soc. 139, 9431-9434 (2017).

57. D. Ron, P. Walter, Signal integration in the endoplasmic reticulum unfolded protein response. Nat. Rev. Mol. Cell Biol. 8, 519-529 (2007).

58. P. Agrawal, B. Fontanals-Cirera, E. Sokolova, S. Jacob, C. A. Vaiana, D. Argibay, V. Davalos, M. McDermott, S. Nayak, F. Darvishian, M. Castillo, B. Ueberheide, I. Osman, D. Fenyö, L. K. Mahal, E. Hernando, A systems biology approach identifies FUT8 as a driver of melanoma metastasis. Cancer Cell 31, 804-819 (2017).

59. E. Lau, Y. Feng, G. Claps, M. N. Fukuda, A. Perlina, D. Donn, L. Jilaveanu, H. Kluger, H. H. Freeze, Z. A. Ronai, The transcription factor ATF2 promotes melanoma metastasis by suppressing protein fucosylation. Sci. Signal. 8, ra124 (2015).

60. M. Aubert, L. Panicot, C. Crotte, P. Gibier, D. Lombardo, M. O. Sadoulet, E. Mas, Restoration of alpha( 1,2$)$ fucosyltransferase activity decreases adhesive and metastatic properties of human pancreatic cancer cells. Cancer Res. 60, 1449-1456 (2000).

61. C. Sun, P. Chen, Q. Chen, L. Sun, X. Kang, X. Qin, Y. Liu, Serum paraoxonase 1 heteroplasmon, a fucosylated, and sialylated glycoprotein in distinguishing early hepatocellular carcinoma from liver cirrhosis patients. Acta Biochim. Biophys. Sin. 44, 765-773 (2012).

62. S. Zhang, K. Jiang, Q. Zhang, K. Guo, Y. Liu, Serum fucosylated paraoxonase 1 as a potential glycobiomarker for clinical diagnosis of early hepatocellular carcinoma using ELISA Index. Glycoconj. J. 32, 119-125 (2015).

63. C. Tu, M. Wu, Y. Lin, R. Kannagi, R. Yang, FUT8 promotes breast cancer cell invasiveness by remodeling TGF- $\beta$ receptor core fucosylation. Breast Cancer Res. 19, 111 (2017).

64. B. W. Mallard, J. Tiralongo, Cancer stem cell marker glycosylation: nature, function and significance. Glycoconj. J. 34, 441-452 (2017).

65. A. Bensadoun, D. Weinstein, Assay of proteins in the presence of interfering materials. Anal. Biochem. 70, 241-250 (1976).

66. J. Cha, P. Kim, Time series assessment of the effects of hypoxic stress on glioma tumorsphere development within engineered microscale niches. Biomaterials 194, 171-182 (2019).

67. R. J. Boado, Y. Zhang, Y. Zhang, Y. Wang, W. M. Pardridge, IgG-paraoxonase-1 fusion protein for targeted drug delivery across the human blood-brain barrier. Mol. Pharm. 5, 1037$1043(2008)$.

68. M. B. D. Aldonza, J. Ku, J. Hong, D. Kim, S. J. Yu, M. Lee, M. C. Prayogo, S. Tan, D. Kim, J. Han, S. K. Lee, S. G. Im, H. S. Ryu, Y. Kim, Prior acquired resistance to paclitaxel relays diverse EGFR-targeted therapy persistence mechanisms. Sci. Adv. 6, eaav7416 (2020).

69. M. B. D. Aldonza, R. D. Delos Reyes, Y. S. Kim, J. Ku, A. M. Barsallo, J. Y. Hong, S. K. Lee, H. S. Ryu, Y. Park, J. Y. Cho, Y. Kim, Chemotherapy confers a conserved secondary tolerance to EGFR inhibition via AXL-mediated signaling bypass. Sci. Rep. 11, 8016 (2021).

70. C. Xu, S. Wang, G. Thibault, D. T. W. Ng, Futile protein folding cycles in the ER are terminated by the unfolded protein O-mannosylation pathway. Science 340, 978-981 (2013).

71. E. T. Elkiran, N. Mar, B. Aygen, F. Gursu, A. Karaoglu, S. Koca, Serum paraoxonase and arylesterase activities in patients with lung cancer in a Turkish population. BMC Cancer 7, 48 (2007).

72. S. Tyanova, T. Temu, J. Cox, The MaxQuant computational platform for mass spectrometrybased shotgun proteomics. Nat. Protoc. 11, 2301-2319 (2016).

73. D. Bach, D. Kim, S. Y. Bae, W. K. Kim, J. Hong, H. Lee, N. Rajasekaran, S. Kwon, Y. Fan, T. Luu, Y. K. Shin, J. Lee, S. K. Lee, Targeting nicotinamide N-methyltransferase and miR-449a 
in EGFR-TKI-resistant non-small-cell lung cancer cells. Mol. Ther. Nucleic Acids 11, 455-467 (2018).

74. D. Kim, D. Bach, Y. Fan, T. Luu, J. Hong, H. J. Park, S. K. Lee, AXL degradation in combination with EGFR-TKI can delay and overcome acquired resistance in human non-small cell lung cancer cells. Cell Death Dis. 10, 361 (2019).

75. D. Bach, T. Luu, D. Kim, Y. J. An, S. Park, H. J. Park, S. K. Lee, BMP4 upregulation is associated with acquired drug resistance and fatty acid metabolism in EGFR-mutant nonsmall-cell lung cancer cells. Mol. Ther. Nucleic Acids 12, 817-828 (2018).

\section{Acknowledgment}

We thank the members of the Yoosik Kim and Je-Yoel Cho labs for support and feedback. We are grateful to Thannaree Chottitisupawong for establishing lapatinib- and PHA605752resistant cell lines; Jung-Mo Ahn for help with N-glycosylation peptide mapping; Stephanie Tan and Monica Prayogo for support in secretome preparation; Sang Kook Lee for PC9- and HCC827-derived cell lines; Eui-Cheol Shin, Eugene Cho, David Helfman, Seung-Jae Lee, Ki Jun Jeong, and Jinyoung Kang for critical kits, reagents, antibodies, and plasmids; core FACS and confocal microscopy facilities of Seoul National University College of Pharmacy, KAIST Biological Sciences, and KAIST GSMSE for technical support. We also thank the glycobiology Twitter (\#glycotime) and Open Memeing Frame communities for open discussion and experimental advice.

\section{Funding}

This research was supported by the KAIST College of Engineering Global Initiative Convergence Research (grant no. N11190234), the Basic Science Research Program (grant nos. NRF-2019R1C1C1006672 and NRF-2018R1A6A3A01012494), the Bio \& Medical Technology Development Program (grant no. NRF-2016M3A9B6026771) through the National Research Foundation (NRF) funded by the Korean government's Ministry of Science and ICT, and the Korea Health Technology R\&D Project through the Korea Health Industry Development Institute (KHIDI), funded by the Korean government's Ministry of Health \& Welfare (grant no. HI14C1324). M.B.D.A. is supported by the Hyundai Motor Chung MongKoo Foundation Global Scholarship (FHS-20-008).

\section{Author contributions}

M.B.D.A. conceived the project, designed, performed, and analyzed most of the experiments, and wrote the manuscript with Yoosik K.; J.C. and I.Y. established the tumor spheroid culture, performed and analyzed live cell imaging experiments; J.K. analyzed RNA-seq data and helped with data analysis; P.S. analyzed LC-MS/MS data; D. L., D. K., and M.B.D.A. performed LC-MS/MS experiments; R.D.D.R. assisted in computational and data analysis using public datasets; R.E.C., M.K., and Yongsuk K. generated stable RFP cells and assisted in fucosylation experiments; H.J.S. and M.B.D.A. generated all PON1-modified cell lines and established the LLC metastasis model; Soyeon K. and T.M.K. prepared patient serum samples and established some resistant cell lines; G.P. assisted in RNA work; T.M.K., S.C., and H.S.R. provided and curated all IRB-approved human cancer patient specimens; Yoosik K., J.Y.C., and P.K. supervised the project.

\section{Competing interests}

Yoosik K., M.B.D.A., P.K., J.C., I.Y., and J.Y.C. have filed a patent for the biomarker signature revealed in this study. J.Y.C. is CEO of ProtanBio Inc., a disease biomarker venture company 
of Seoul National University. H.J.S. was employed in the same company. The authors declare that they have no other competing interests.

\section{Data and materials availability}

All sequencing data produced for this publication has been deposited to the NCBI Gene Expression Omnibus (GEO) database under the accession number GSE160205. The mass spectrometry proteomics data have been deposited to the ProteomeXchange Consortium via the PRIDE partner repository with the dataset identifier PXD022240. Other data associated with this study are present in the paper, Supplementary Materials, or source data files. Additional data related to this paper may be requested from M.B.D.A., Yoosik K., or J.Y.C. Reagents and cell lines described here are accessible through a materials transfer agreement.

\section{Code availability}

The main scripts used for data analyses and plotting are described in detail and are available upon request from M.B.D.A. or from https://github.com/borrisHUBO/Aldonza-et-al.-NatureCommunications.

\section{Figure description}

Fig. 1. Secretome fucosylation is a post-translational mechanism associated with targeted therapy resistance in cancer.

(A) Heat-scatterplot visualization of correlation between indicated FUT gene expression and drug response per cancer type screened in GDSC and CCLE. Per-sample estimates of area under the fitted dose response curve were used as metric of drug response per cell line. Size of circle refers to mean log2 gene expression while color corresponds to Spearman's rank coefficients. Only statistically significant correlations are shown $(P<0.05)$. Beside are relative mean proportion of mutational signatures of all FUT genes per cancer type queried in GDSC and CCLE. FUT mutations were classified as "GDP-Fuc binding site mutations" if any mutations (amino acid change) occurred near ( \pm 5 amino acid position) or at the annotated GDP-Fuc binding sites. Domain information was queried in UniProt. Spearman's rank coefficients (correlation between FUT expression and drug response) were calculated in cell lines carrying these mutations as opposed to those that do not ("others").

(B) Positive correlation between FUT8 gene expression and resistance to drugs grouped accordingly per target process in GDSC. Data from both GDSC and CCLE are summarized. Color represents Spearman's rank coefficients per target process. Only statistically significant correlations are shown $(P<0.01)$. Bars indicate number of drugs per class while size of circle corresponds to relative Spearman's rank coefficients per drug. Beside is a proportion of drug categories (GDSC classification) from all drugs with resistance profiles positively correlated with FUT8 expression.

(C) Heat-scatterplot visualization of differential (TCGA primary tumor versus paired normal) $\mathrm{CCS}$ and overlapped glycosylation gene set expressions (including $\mathrm{O}-\mathrm{N} \mathrm{N}$-linked glycosylation) per cancer type. Size of circle refers to adjusted $-\log 10 p$ value while color corresponds to log2 fold change in expression. Statistically significant $(P<0.05)$ Spearman's correlation between drug sensitivity and CCS or glycosylation expression derived from GDSC are shown as heatmap. In total, 169 drug profiles were queried; 33 are targeted and 10 are cytotoxic drugs. (D) Heat-scatterplot visualization of mean promoter methylation fraction $1 \mathrm{~kb}$ upstream of the TSS of indicated FUT genes per cancer type from CCLE RRBS dataset. Size of circle refers to number of screened cell lines while color corresponds to FUT promoter methylation. Only statistically significant changes are shown $(P<0.05)$. Correlation between drug sensitivity and methylation are shown as heatmap as in $C$.

(E) Schematic of secretome N-glycoprotein core fucosylation.

(F) AAL blot analysis of total fucosylation in indicated crude patient sera prepared as in the left 
panel. Representative of two independent experiments. Equal loading controls and AAL specificity are presented in Supplemental Fig. 4.

(G) N-glycan release assay using indicated $\mathrm{N}$-glycan-cleaving enzymes in crude patient sera prepared as in F. Prior to this assay, samples were separated by SDS-PAGE followed by Coomassie staining. 30 60 kDa in-gel proteins were then excised followed by exogenous de$\mathrm{N}$-glycosylation (total 8U PNGase F or total 10U Endo S/F). Glycan cleavage site is shown for each enzyme. Values indicate mean absorbance at $584 \mathrm{~nm}$ from three replicates. Representative of two independent experiments. Sample size $\mathrm{n}$, unique patient samples. For statistical analysis, two-tailed Mann-Whitney $U$ test was used. NS, not significant.

(H) Preparation of cell secretomes and schematic of sandwich ELLA.

(I) Characterization of fucosylation by sandwich ELLA in indicated secretomes from sensitive cells or DR clones prepared as in $\mathrm{H}$ following treatment with or without indicated drugs for 48 h. Values are relative to DMSO (means \pm SD of three biological replicates). $P$ values are indicated as size of the corresponding circle; Student's $t$-test. NS, not significant.

(J) N-glycan release assay using indicated $\mathrm{N}$-glycan-cleaving enzymes in indicated secretomes from sensitive cells or DR clones prepared as in $\mathrm{H}$ following treatment with or without indicated drugs for $48 \mathrm{~h}$. 30 60 kDa in-gel proteins were then excised followed by exogenous de-N-glycosylation (total $8 \mathrm{U}$ PNGase F or total 10U Endo S/F). Glycan cleavage site is shown for each enzyme. Values indicate mean absorbance at $584 \mathrm{~nm}$ from three replicates. Representative of two independent experiments. For statistical analysis, two-tailed Mann-Whitney $U$ test was used. NS, not significant.

(K) Representative confocal images of indicated DR clones stained for RCAS1 (golgi marker; green), fluorescein-conjugated AAL (core fucosylation; red), and DAPI (nuclei; white). Colocalization histogram plot of indicated line is shown. Representative of two independent experiments.

( $\mathbf{L}$ and $\mathbf{M}$ ) AAL blot analysis of total fucosylation in indicated secretomes from sensitive or resistant cells following treatment with or without indicated drugs for $48 \mathrm{~h}$. Samples were prepared as in $\mathrm{H}$. Representative of two independent experiments. Equal loading controls and AAL specificity are presented in Supplemental Fig. 4.

(N) Characterization of fucosylation by sandwich ELLA in indicated secretomes from sensitive cells or DR clones following treatment with or without respective drug IC50s for $48 \mathrm{~h}$; or sera from patients treated with or without osimertinib. Cell secretomes were prepared as in $\mathrm{H}$ while patient sera were prepared as in F; except filtered according to their indicated nominal molecular weight limit (NMWL). Values are relative to unfiltered secretome/sera (means $\pm S D$ of three biological replicates). $P$ values are indicated as size of the corresponding circle; Student's $t$-test. NS, not significant.

Fig. 2. Secretome fucosylation promotes resistance rebound in regressing cell admixtures.

(A) Schematic of multi-color cell tracker assay in 'one pot' admixture culture.

(B) Representative live-imaging confocal images of indicated 3D tumor spheroid admixture prepared as in A and treated with or without $2 \mu \mathrm{M}$ gefitinib for $24 \mathrm{~h}$. Scale bar indicates 100$\mu \mathrm{m}$. Mean intensity profiles of both fluorescently-tagged cells are shown. See also Supplemental Movies 1, 2 and 3.

(C) Representative Coomassie stained SDS-PAGE gels showing fucosylated secretome proteins from indicated $3 \mathrm{D}$ cell admixtures prepared as in $\mathrm{A}$, treated with $2 \mu \mathrm{M}$ gefitinib or 0.1 $\mu \mathrm{M}$ erlotinib for 1 or 5 day/s, and incubated with or without $10 \mu \mathrm{g} / \mathrm{mL}$ recombinant PNGase $\mathrm{F}$. Secretomes were concentrated using a $>3 \mathrm{kDa}$ NMWL filter. Representative of two independent experiments.

(D) Characterization of fucosylation by sandwich ELLA in indicated cell admixture secretomes with conditions as in $\mathrm{C}$. Values are relative to non-treated secretome (means $\pm \mathrm{SD}$ of three biological replicates). ${ }^{* * *} P<0.001$, Student's $t$-test.

(E) Characterization of fucosylation by AAL blotting, sandwich ELLA, and N-glycan release assay in indicated 2D cell admixtures prepared as in $A$, treated with or without $1 \mu \mathrm{M}$ gefitinib 
or $0.1 \mu \mathrm{M}$ erlotinib, and incubated with or without $10 \mu \mathrm{g} / \mathrm{mL}$ recombinant PNGase $\mathrm{F}$ for up to 5 days. Secretomes were concentrated using a $>30 \mathrm{kDa}$ NMWL filter. Blots are representative of two independent experiments. Values are relative to day 0 (means \pm SD of two biological replicates). ${ }^{* *} P<0.01$, Student's $t$-test.

(F) Representative confocal images of fluorescently-tagged GR clone in 3D cell admixtures prepared as in A, treated with $2 \mu \mathrm{M}$ gefitinib, and incubated with or without $10 \mu \mathrm{g} / \mathrm{mL}$ recombinant PNGase F for 24 or $48 \mathrm{~h}$. Scale bar indicates $100-\mu \mathrm{m}$. Intensity profiles of trackertagged GR clone are shown. Values are relative to day 0 (means \pm SD of three biological replicates). ${ }^{* * *} P<0.001$, Student's $t$-test. NS, not significant.

(G) Tracking of both fluorescently-tagged cells in 2D cell admixtures prepared as in A, treated with or without $1 \mu \mathrm{M}$ gefitinib, and incubated with or without $10 \mu \mathrm{g} / \mathrm{mL}$ recombinant PNGase $\mathrm{F}$ for indicated times. Values are relative to day 0 (means \pm SD of three biological replicates). Beside shows cell cycle states of adherent cells and apoptosis of floating cells in indicated cell admixtures with same conditions at day 5 . Cell cycle assays are representative of two independent experiments. ${ }^{* *} P<0.01,{ }^{* * *} P<0.001$, two-tailed Mann-Whitney $U$ test. NS, not significant.

(H) Similar tracking experiments as in G, except upon FUT8 or SLC35C1 RNAi in sensitive cells for $48 \mathrm{~h}$ prior to admixing and culture for 5 days. $\mathrm{H} 1993$ admixture was treated with or without $1 \mu \mathrm{M}$ gefitinib, PC9 admixture was treated with or without $0.1 \mu \mathrm{M}$ erlotinib, and A375 admixture was treated with or without $0.1 \mu \mathrm{M}$ vemurafenib. Values are relative to day 0 (means \pm SD of two biological replicates). ${ }^{* *} P<0.01$, ${ }^{* * *} P<0.001$, two-tailed Mann-Whitney $U$ test. NS, not significant. PNGase F controls are presented in Supplemental Fig. 14E.

(I) Characterization of fucosylation by sandwich ELLA in indicated apoptotic debris and secretomes from the same cell admixtures as in $\mathrm{G}$. Values are relative to control apoptotic debris (means $\pm \mathrm{SD}$ of three biological replicates). ${ }^{* * *} P<0.001$, Student's $t$-test. NS, not significant.

(J) Phospho-RTK array of indicated cell admixtures in the same conditions as in G. The blots reflect the phosphorylation status of 49 RTKs. Each RTK is spotted in duplicate, and the three pairs of dots in each corner are positive or negative controls. Representative of two independent experiments.

(K) Schematic of CM co-culture.

(L) Colony formation of indicated DR clones prepared as in K. Representative of two independent experiments.

(M) ELISA sandwich-based measurement of indicated RTK phosphorylation in indicated DR clones prepared as in K. Values are relative to DMSO (means \pm SD of three biological replicates). ${ }^{* *} P<0.01,{ }^{* * *} P<0.001$, Student's $t$-test.

(N) qPCR analysis of indicated gene expression in 3D cell admixtures prepared as in A, treated with or without $2 \mu \mathrm{M}$ gefitinib, and incubated with or without $10 \mu \mathrm{g} / \mathrm{mL}$ recombinant PNGase $F$ for 5 days. Values are relative to DMSO and were normalized to GAPDH levels (means \pm SD of three biological replicates). $P$ values are indicated as size of the corresponding circle; Student's $t$-test. NS, not significant.

\section{Fig. 3. Identification of fucosylated PON1 as a critical component of therapy-induced cancer secretomes.}

(A) Schematic of label-free secretome analysis workflow.

(B) GO enrichment analysis for overrepresented BPs in cell-specific secretomes. Fold enrichment is shown as heatmap. - $\log 10 p$ values (red), false discovery rates (green), and number of gene components per BP (gray) are displayed. Results were analyzed from two biological replicates.

(C) Protein candidate screening approach and log10 LFQ intensities (relative protein abundances) of indicated overlapped proteins in secretomes of both gefitinib-treated $\mathrm{H} 1993$ cells and GR clone. Top 11 protein hits with MWs between 30 and $70 \mathrm{kDa}$ are shown. Results were analyzed from two biological replicates.

(D) Immunoblot and AAL blot analyses of PON1 expression and fucosylation status in PON1 
immunoprecipitates from $1 \mu \mathrm{M}$ gefitinib-treated H1993 secretomes. Secretomes were exogenously treated with or without $8 \mathrm{U}$ PNGase F. Bottom panel shows glycoprotein stained SDS-PAGE gel of the same PON1 immunoprecipitates. Representative of two independent experiments.

(E) Schematic of HLE for detecting PON1 fucosylation.

(F) HLE analysis of PON1 fucosylation in secretomes from indicated cells and DR clones treated with or without indicated drug concentrations for $48 \mathrm{~h}$. Values are relative to DMSO or parental (means \pm SD of three biological replicates). $P$ values are indicated as size of the corresponding circle; Student's $t$-test. NS, not significant.

(G) Immunoblot analysis of PON1 expression in indicated crude patient sera exogenously treated with or without $8 U$ PNGase F. Secretomes were either flow-through or enriched in $A A L$ columns. Representative of two independent experiments. (H) AAL blot analysis of PON1 fucosylation in PON1 immunoprecipitates from indicated patient sera. Representative of two independent experiments.

(I) HLE analysis of PON1 fucosylation in indicated crude patient sera. Values indicate mean absorbance at $450 \mathrm{~nm}$ from three replicates. Representative of two independent experiments. Beside shows quantification of paraoxonase activity in the same crude patient sera. Values indicate mean fluorescence units at $412 \mathrm{~nm}$ from three replicates. Representative of two independent experiments. ROC curves for both PON1 fucosylation and paraoxonase activity are shown. For statistical analysis, nonparametric Kruskal-Wallis test was used.

(J) Representative confocal images of indicated DR clones stained for RCAS1 (golgi marker; green), PON1 (red), and DAPI (nuclei; white). Co-localization histogram plot of indicated line is shown. Representative of two independent experiments.

(K) AAL blot analysis of PON1 fucosylation in PON1 immunoprecipitates from indicated subcellular fractionated H1993-GR. Middle panel shows glycoprotein stained SDS-PAGE gel of subcellular fractionated cell lysates. Bottom panel shows immune blot analysis of RCAS1 in the same cell lysates. Representative of two independent experiments.

(L) HLE analysis of PON1 fucosylation in indicated subcellular fractionated DR clone lysates upon SLC35C1 RNAi for $48 \mathrm{~h}$. Values indicate absorbance at $450 \mathrm{~nm}$ (means \pm SD of three biological replicates). ${ }^{* * *} P<0.001$, Student's $t$-test. NS, not significant.

(M) Representative confocal images of H1993-GR upon SLC35C1 RNAi for $48 \mathrm{~h}$. GR clones were stained for SLC35C1 (white) and DAPI (nuclei; blue).

(N) Genes co-expression network of PON1 queried in the CCLE. All nodes represent statistically significant co-expression with a gene. Top 20 PON1 co-expressing genes are highlighted. Colored nodes indicate cellular localization of protein-coding genes queried in The Human Protein Atlas.

(O) Representative confocal images of indicated DR clones stained for PON1 (red), PON3 (green), and DAPI (nuclei; white). Co-localization histogram plot of indicated line is shown.

(P) HLE analysis of PON1 fucosylation and quantification of paraoxonase activity in Golgi/ER fractionated H1993-GR lysates upon SLC35C1, PON1, or PON3 RNAi for 48 h. Values are relative to siControl (means $\pm S D$ of three biological replicates). ${ }^{* *} P<0.001$, Student's $t$-test. NS, not significant.

(Q) GDP-Fuc activity analysis of FUT8 in cross-linked FUT8 and PON1 co-immunoprecipitates from Golgi/ER fractionated H1993-GR lysates. Values indicate luminescence units and are relative to control reaction (means $\pm S D$ of three biological replicates). ${ }^{*} P<0.05,{ }^{* *} P<0.001$, two-tailed Mann-Whitney $U$ test.

Fig. 4. Core fucosylation impacts PON1 folding and stability prior to secretion in therapy-resistant cancer cells.

(A) Hypothetical model of $\mathrm{N}$-glycosylation control of PON1 stability.

(B) Full-length PON1 N-glycosylation site prediction using NetNGlyc 1.0, folding prediction using Foldlndex, and charge prediction using EMBOSS. $>0.5$ threshold score means significant glycosylation potential. Unfolded regions are depicted in red, folded regions in green. Positive charged is marked in red shades, negative charge in blue, and neutral charge 
in white.

(C) Conservation of indicated PON1 sequons throughout species.

(D) Closed conformation surface structure of PON1 (PDB ID: 1V04) highlighting arylesterase domain and predicted N-glycosylation sites and sequons. 3D surface view was visualized using PyMOL.

(E) N-glycan structural analysis of PON1 from our previous tandem MS/MS dataset. The $\mathrm{m} / \mathrm{z}$ 1,647.62 [(M+Na)+corresponding to GlcNAc2Man3+HexNAc2Hex1Fuc1] is the base peak (not visualized). Putative structure visualization of indicated monosaccharides and FUT8 substrate specificity were based on CID data and known glycobiology.

(F) Prediction of PON1 stability, structural and functional properties upon indicated in silico $\mathrm{N} \rightarrow \mathrm{G}$ substitution at specific sequons using MutPred 2.0 and I-Mutant 3.0. Two $\mathrm{N} \rightarrow \mathrm{G}$ substituted sequons (N253G and N324G) with statistically significant potential of loss of Nglycosylation were chosen for validation experiments.

(G) AAL blot analysis of PON1 immunoprecipitates from H1993-GR upon transfection with indicated full-length PON1, PON1-N253G, or PON1-N324G constructs for $36 \mathrm{~h}$. Representative of two independent experiments. Beside shows HLE analysis of secretome PON1 fucosylation and N-glycan release assay in AAL-enriched PON1 immunoprecipitates from H1993-GR upon similar transfection. Values are relative to full-length PON1 (means \pm SD of three biological replicates). ${ }^{* *} P<0.001$, Student's $t$-test.

(H) GDP-Fuc activity analysis of FUT8 in cross-linked FUT8 and PON1 co-immunoprecipitates from H1993-GR upon transfection with constructs as in G. Values indicate luminescence units and are relative to control reaction (means $\pm S D$ of three biological replicates). ${ }^{* \star} P<0.01$, ${ }^{* * *} P<0.001$, two-tailed Mann-Whitney $U$ test.

(I) Immunoblot analysis of PON1 expression in H1993-GR upon transfection with constructs as in G. Lysates were exogenously treated with or without indicated trypsin concentration. Representative of two independent experiments.

(J) ELISA analysis of PON1 expression in H1993-GR upon transfection with constructs as in G. Golgi/ER fractionated cell lysates were exogenously treated with or without indicated trypsin concentrations. Values are relative to no treatment (means $\pm S D$ of three biological replicates). ${ }^{*} P<0.05,{ }^{* *} P<0.01,{ }^{* *} P<0.001$, Student's $t$-test. NS, not significant.

(K) EZClick labeling analysis of polypeptide synthesis in H1993-GR upon transfection with constructs as in $\mathrm{G}$ and treated with or without $25 \mu \mathrm{g} / \mathrm{mL} \mathrm{CHX}$ concentrations for indicated times. Values indicate raw fluorescence units (means \pm SD of two biological replicates). For statistical analysis, Student's $t$-test was used. NS, not significant.

(L) Immunoblot analysis of PON1 expression in H1993-GR upon transfection with constructs as in $\mathrm{G}$ and treated with or without $25 \mu \mathrm{g} / \mathrm{mL} \mathrm{CHX}$ for indicated times. GAPDH was used as a loading control. Blot intensity quantification of the lower PON1 kDa isoform is shown. Representative of two independent experiments.

(M) ELISA analysis of secretome PON1 expression in H1993-GR upon transfection with constructs as in $\mathrm{G}$. Values are relative to full-length (means \pm SD of three biological replicates). ${ }^{*} P<0.05,{ }^{* *} P<0.001$, Student's $t$-test.

\section{Fig. 5. Secretome PON1 fucosylation promotes resistance via neutralization of} inflammatory response and ROS.

(A) Cignal 45-pathway array of reporter transcriptional activities in indicated cell admixtures treated with $1 \mu \mathrm{M}$ gefitinib and incubated with or without $10 \mu \mathrm{g} / \mathrm{mL}$ recombinant PNGase $\mathrm{F}$ for 5 days. Log 2 values were normalized by control condition and represented as fold changes in luciferase units (means \pm SD of two biological replicates). Highlighted top up-/down-regulated hits are all statistically significant $(P<0.001$, Dunnett's test).

(B) qPCR analysis of indicated gene expression in 3D cell admixtures with same conditions as in $\mathrm{A}$, except treated with $2 \mu \mathrm{M}$ gefitinib for 2 or 5 days. Values are relative to day 0 control and were normalized to GAPDH levels (means \pm SD of three biological replicates). $P$ values are indicated as size of the corresponding circle; Student's $t$-test. NS, not significant.

(C) qPCR analysis of indicated gene expression in 3D cell admixtures with same conditions 
as in B upon transfection with full-length PON1 or PON1-N253G construct for $36 \mathrm{~h}$. Values are relative to full-length and were normalized to GAPDH levels (means \pm SD of three biological replicates). $P$ values are indicated as size of the corresponding circle; Student's $t$-test. NS, not significant.

(D) Representative confocal images of H1993-GR grown for 5 days in indicated secretomes from $1 \mu \mathrm{M}$ gefitinib-treated $\mathrm{H} 1993$ cells exogenously treated with total 8U PNGase $\mathrm{F}$ or transfected with full-length PON1 or PON1-N253G construct for $36 \mathrm{~h}$. GR clones were stained for fluorescein-conjugated AAL (core fucosylation; green), ATF6 (red), and DAPI (nuclei; blue). Beside shows ELISA analysis of ATF6 expression in Golgi/ER fractionated H1993-GR with the same conditions. Values are relative to full-length (means \pm SD of three biological replicates). ${ }^{* *} P<0.01,{ }^{* * *} P<0.001$, Student's $t$-test. C. Values are relative to day 0 or full-length (means \pm SD of two biological replicates). ${ }^{* *} P<0.01$, ${ }^{* * *} P<0.001$, Student's $t$-test. NS, not significant. secretomes from PON1-N253G-transfected H1993 cells treated with or without $1 \mu \mathrm{M}$ gefitinib for $72 \mathrm{~h}$. Values are relative to DMSO siControl (means \pm SD of two biological replicates). ${ }^{* * *} P<0.001$, Student's $t$-test. NS, not significant.

(G) Schematic of sequentially layered admixture.

(H) Tracking of RFP-tagged H1993-GR upon ATF6 RNAi in 3D cell admixtures as in G. Sensitive cells were transfected with full-length PON1 or PON1-N253G for $36 \mathrm{~h}$. Values are relative to day 0 (means \pm SD of two biological replicates). ${ }^{*} P<0.05,{ }^{* *} P<0.01,{ }^{* * *} P<0.001$, two-tailed Mann-Whitney $U$ test. NS, not significant. prepared as in $D$, except in $2 D$. Values are relative to siControl full-length (means $\pm S D$ of two biological replicates). ${ }^{*} P<0.05,{ }^{* * *} P<0.001$, Student's $t$-test. NS, not significant. (J) Modified secretomes from PON1-edited cells with varying PON1 fucosylation.

(K) ROS/RNS detection in secretomes described as in J. Values are relative to control (means \pm SD of three biological replicates). ${ }^{*} P<0.05,{ }^{* *} P<0.01$, ${ }^{* * *} P<0.001$, Student's $t$-test.

(L) Sandwich ELISA analysis pf indicated cytokines in secretomes described as in J. Values are relative to control/shControl (means \pm SD of two biological replicates). ${ }^{*} P<0.05,{ }^{* *} P<0.01$, ${ }^{* * *} P<0.001$, Student's $t$-test. NS, not significant.

(M) Tracking of RFP-tagged H1993-GR in 3D cell admixtures described in the schematic. Admixtures were grown in secretomes described as in $\mathrm{J}$. Values are relative to day 0 (means $\pm \mathrm{SD}$ of two biological replicates). ${ }^{*} P<0.05,{ }^{* *} P<0.01$, ${ }^{* * *} P<0.001$, two-tailed Mann-Whitney $U$ test. NS, not significant.

(N) Caspase activity analysis in 3D cell admixtures as in $M$ and grown in secretomes described as in $\mathrm{J}$ for 5 days. Values are relative to control/shControl (means \pm SD of two biological replicates). ${ }^{* * *} P<0.001$, Student's $t$-test.

Fig. 6. Transcriptome-wide analysis reveals modulator genes associated with secretome PON1 fucosylation-induced therapy resistance.

(A) Schematic of co-culture conditions and preparation of transcript library from H1993-GR for RNA-seq.

(B) GO analysis of gene expression changes in H1993-GR grown in indicated conditions showing enriched GO terms. Size of circle indicates frequency of the GO term in the underlying GOA database while color indicates adjusted $-\log 10 p$ value. Highly similar GO terms are linked by edges in the graph, where the line width indicates the degree of similarity.

(C) Volcano plots showing differentially expressed genes deregulated by indicated conditions. Significantly up-regulated genes are in red, while down-regulated genes in blue.

(D) Venn diagram indicating overlap of up-regulated or down-regulated genes in indicated conditions.

(E) Log2 fold changes and - $\log 10 p$ values of indicated top 20 overlapped up-regulated or down-regulated genes in indicated conditions as in D. Data are means. $P$ values were 
2094 calculated using a two-tailed Mann-Whitney U test.

(F) Violin plots depicting dependency scores of indicated top differentially expressed genes from two conditions as in D. Scores reflect data from 23 different cancer lineages. Central lines indicate median. Data was obtained from DepMap RNAi screen. Beside shows heatscatterplot visualization of correlation between indicated pan-cancer gene dependency and drug response screened in GDSC. Size of circle refers to linear regression $p$ value while color corresponds to Spearman's rank coefficients.

2101 (G) Kaplan-Meier plots of FP or RFS in multiple lung cancer patient cohorts. Patient survival data were stratified by indicated gene expression (low or high) in their primary tumors based on microarray (FP) or RNA-seq (RFS) data. $P$ values were calculated using a log-rank test. 


\section{Figure 1.}
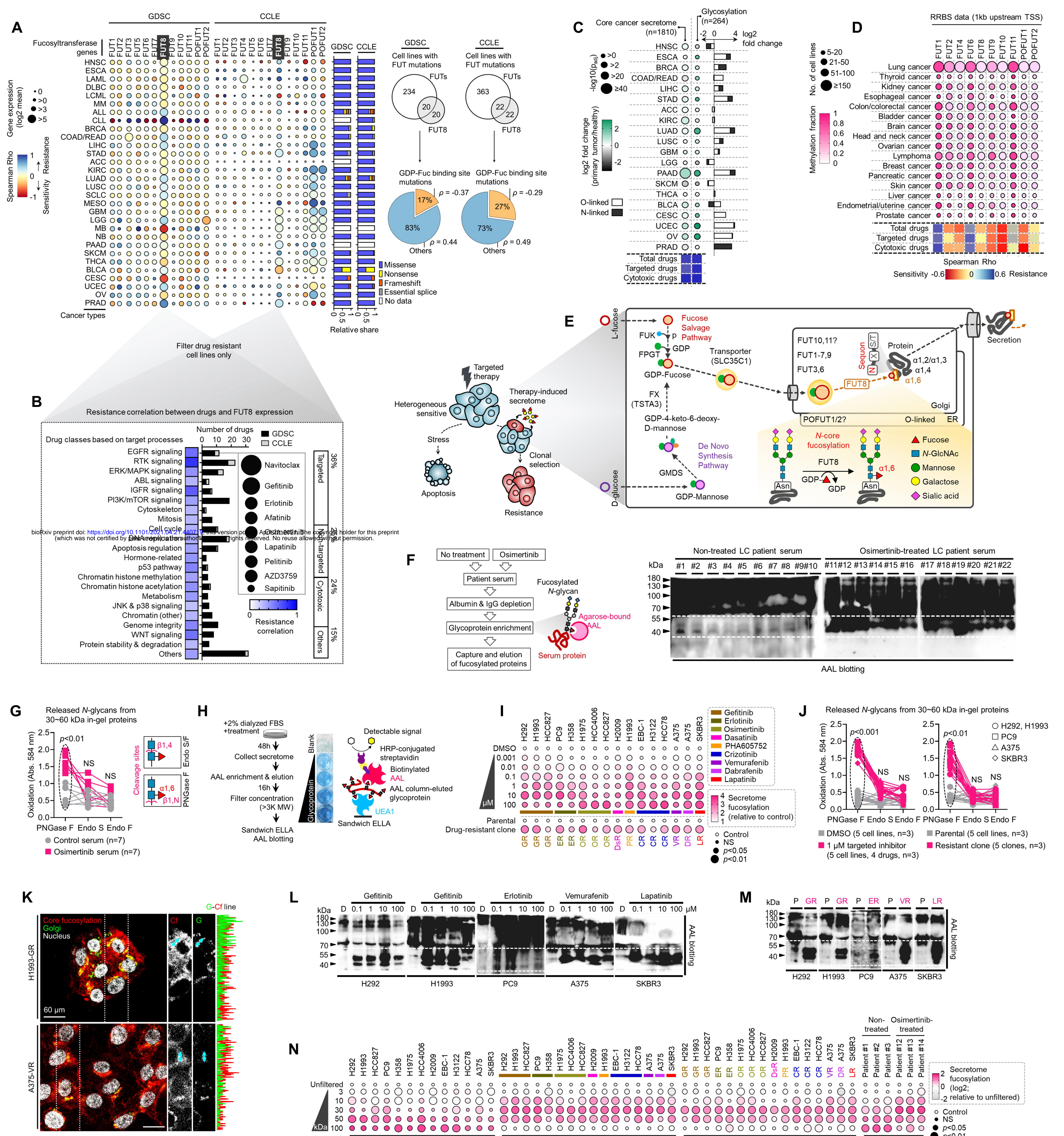
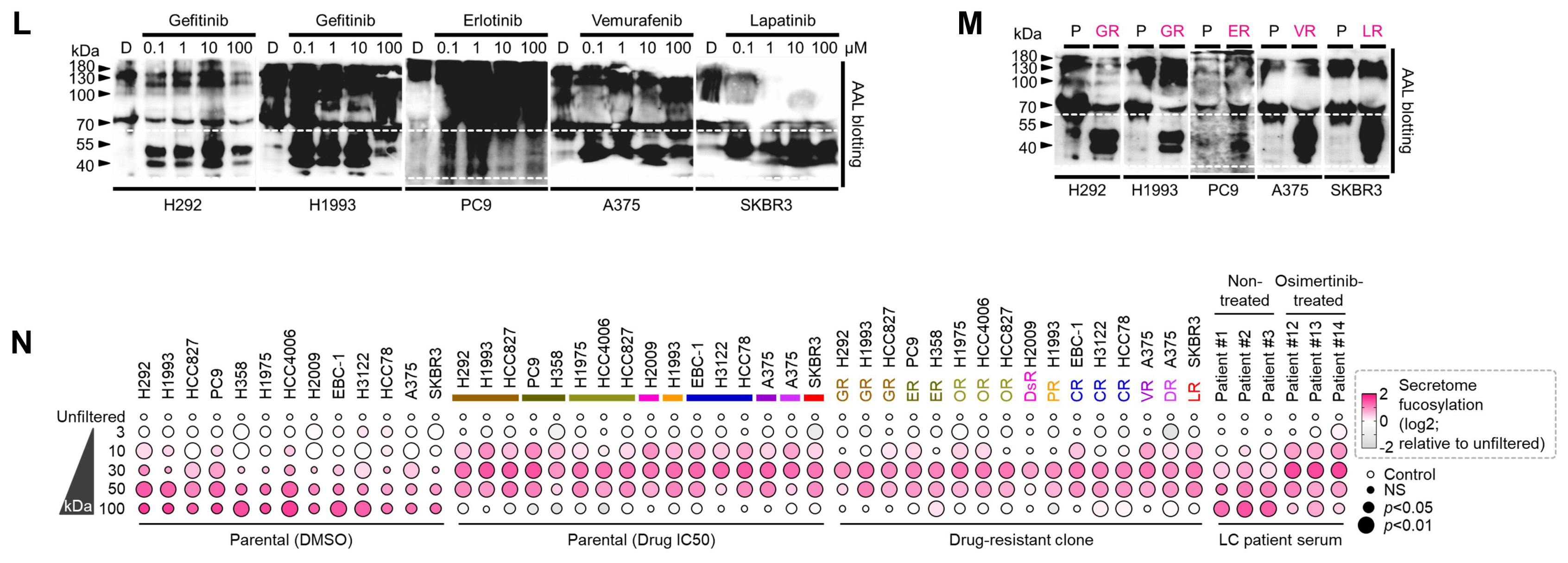


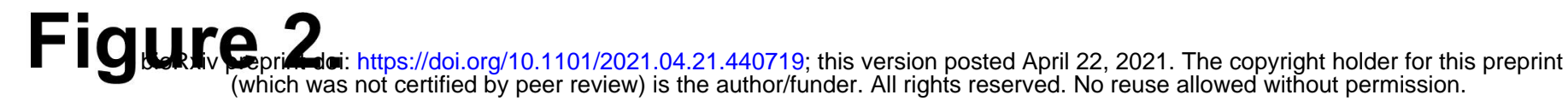

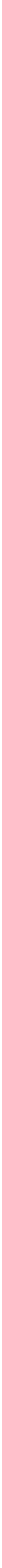




\section{Figure 3.}
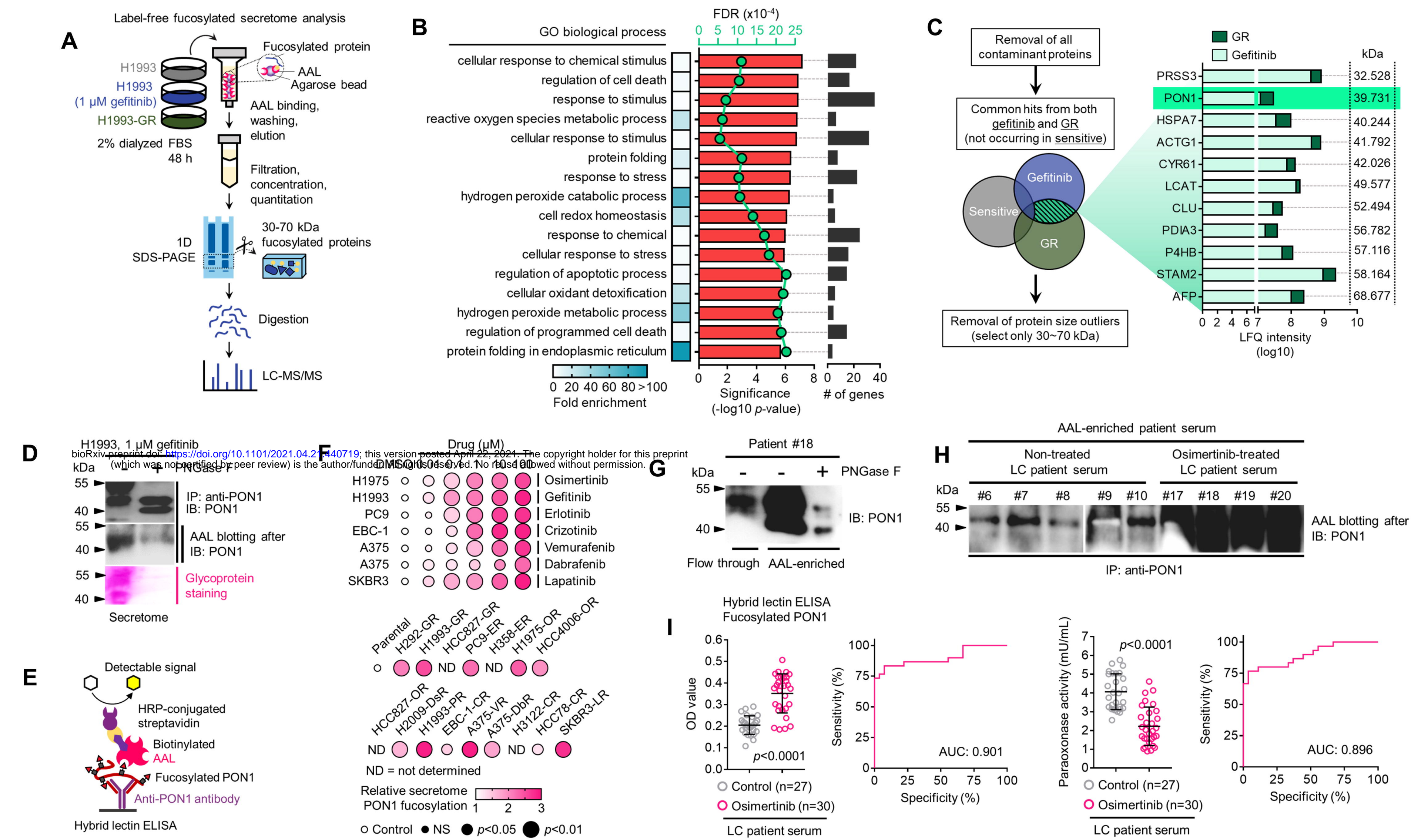

$$
\text { Hybrid lectin ELISA }
$$$$
\text { o Control •NS } p<0.05 \bigcirc p<0.01
$$
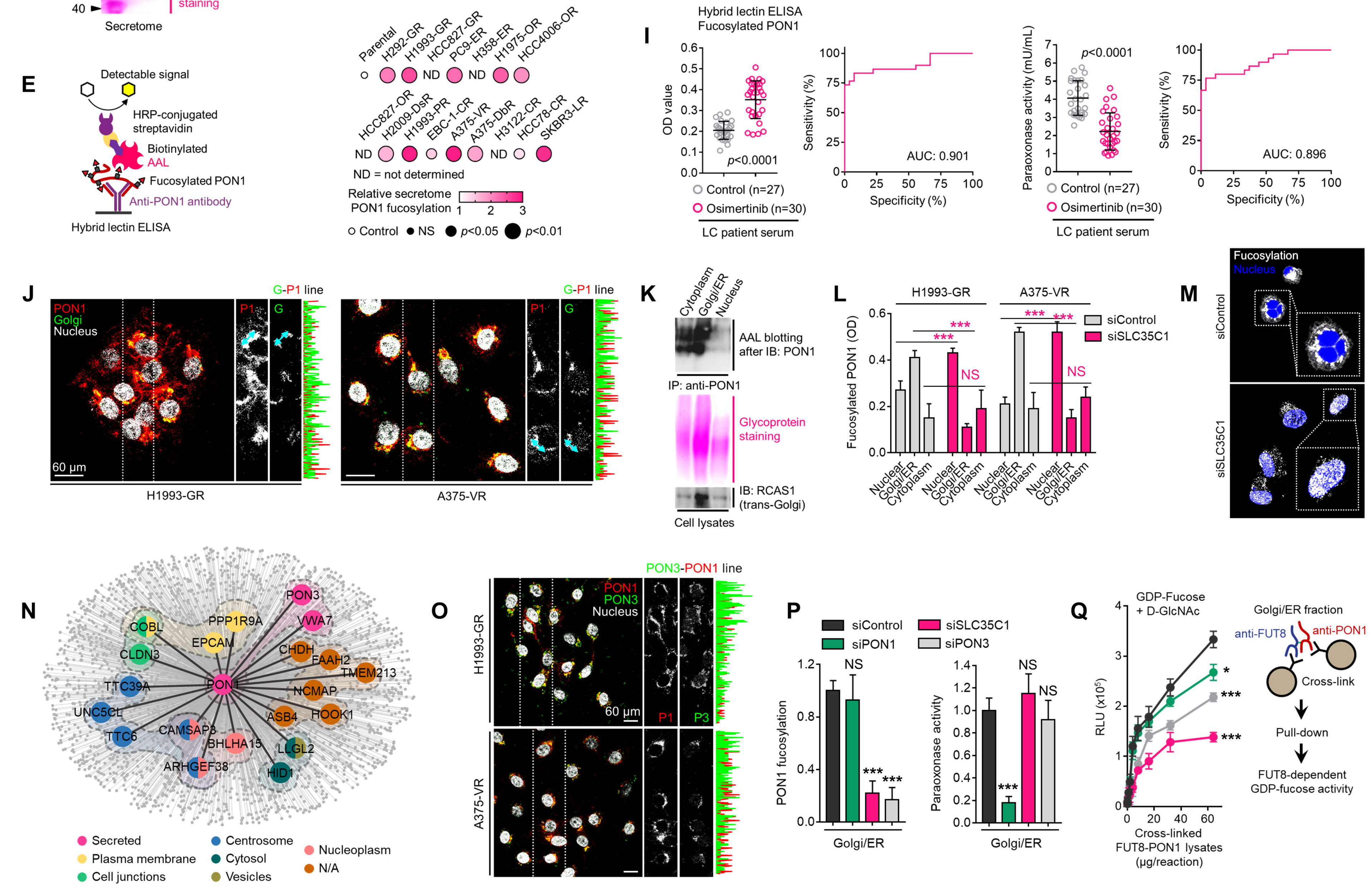

\footnotetext{
$\begin{array}{lll}\text { Plasma membrane } & \text { Cytosol } \\ \text { Cell junctions } & \text { Vesicles }\end{array}$
}
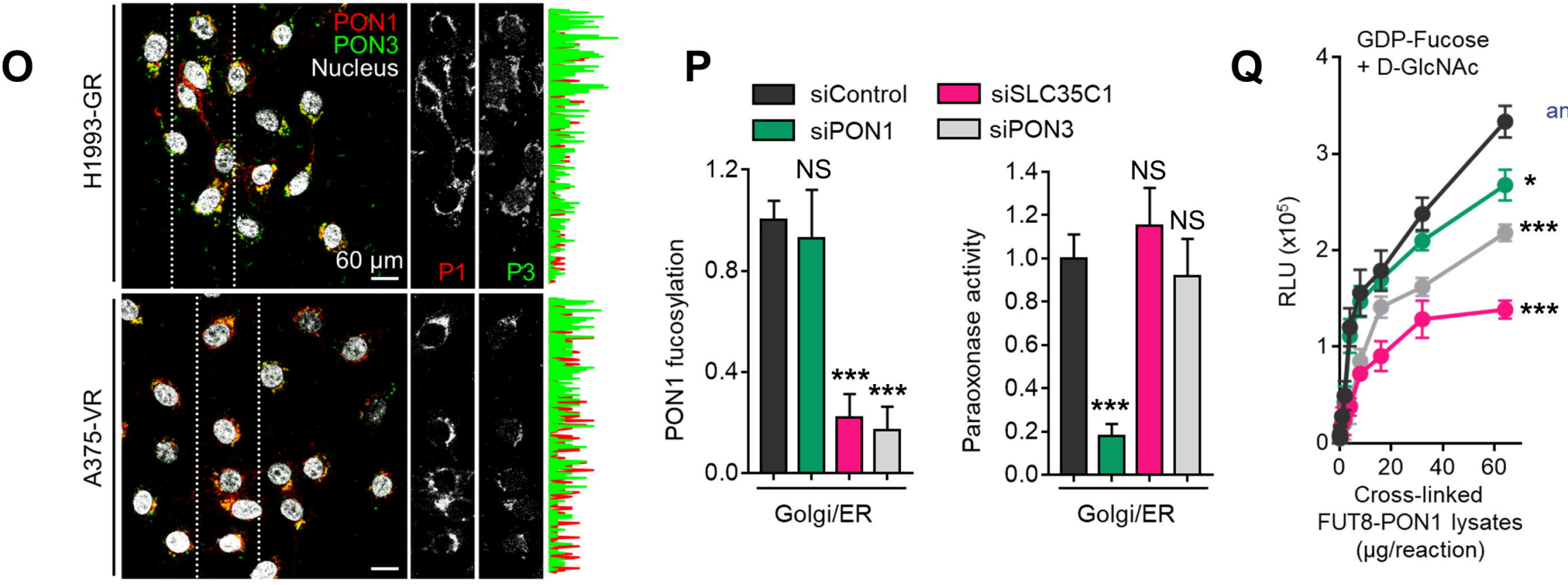

Golgi/ER fraction

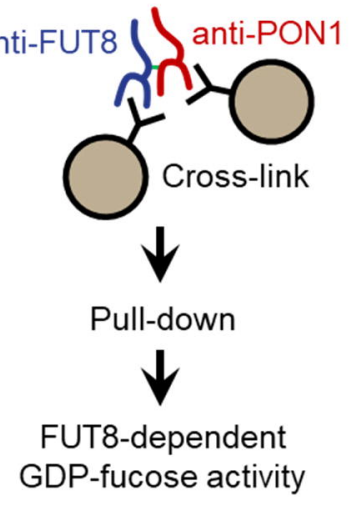


Figure 4.
A

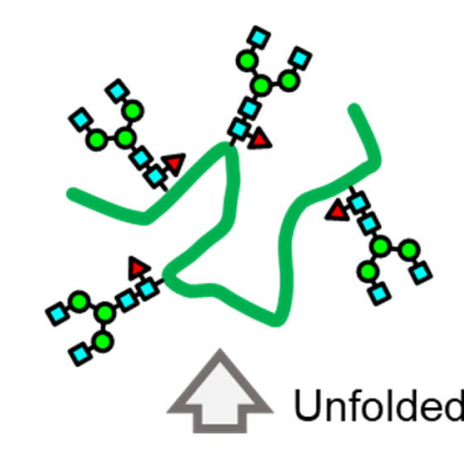

PON1 Folding,

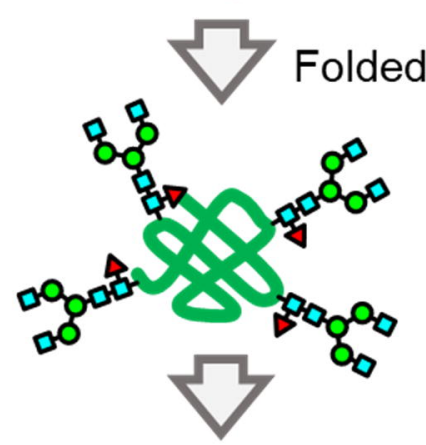

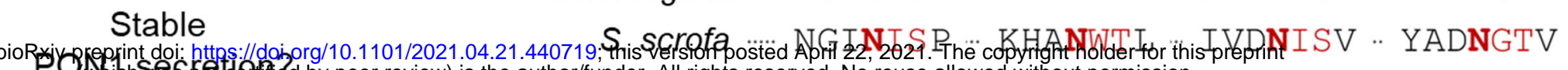

B

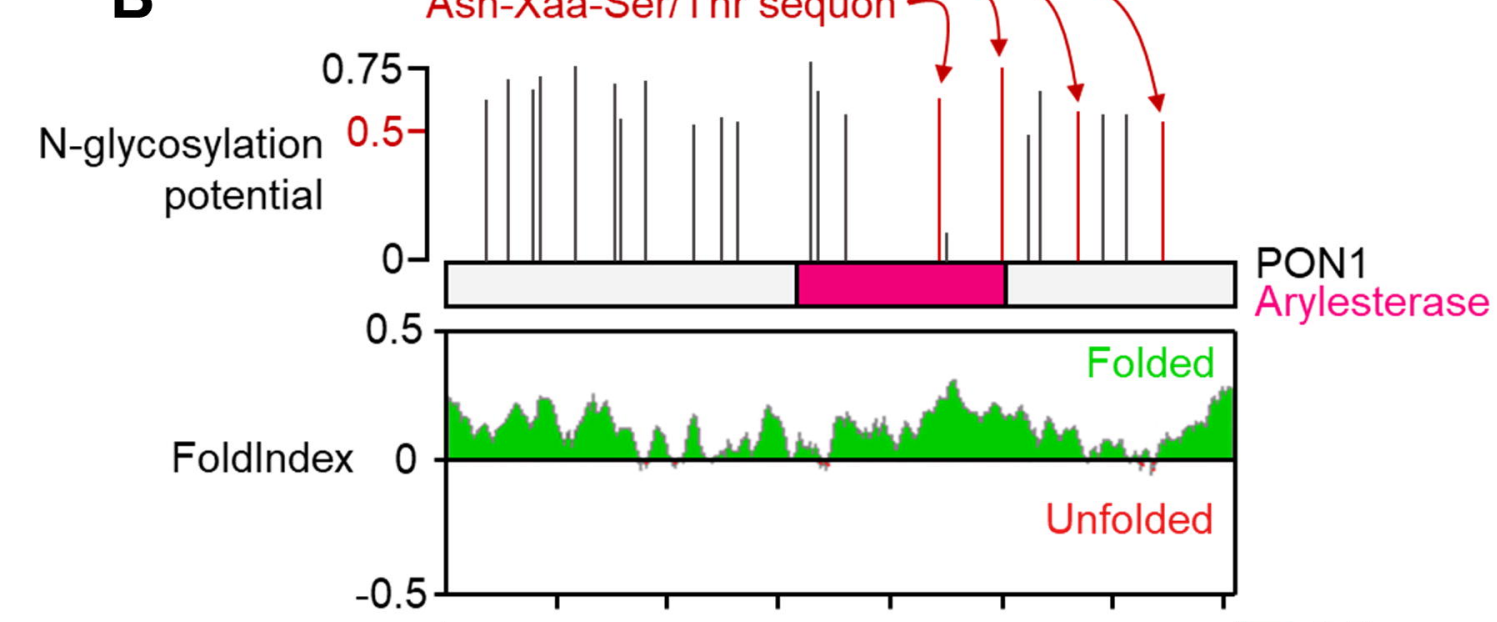

C

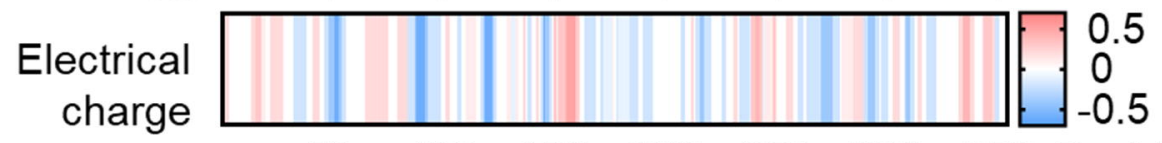

$\begin{array}{llllllll}50 & 100 & 150 & 200 & 250 & 300 & 350 & \text { Residue (aa) }\end{array}$

H. sapiens …. NGINISP … KHANWTL … LVDNISV .. YAENGTV P. troglodytes .... NGINISP ... KHANWTL … LVDNISV .. YAENGTV M. musculus ..... NGIGISL ... KHANWTL ... LVDNISV .. YAENGTV R. norvegicus .... NGIGISL ... KHANWTL ... LVDNISV .. YAENGTV X. tropicalis …. NGIAMST … KQADWSL … LLDNLSV .. YVNNGTV

D. rerio … NGINLSL … IKKNTVL … LADNIEL .. YSDNGSV

T. rubripes …. NGINISP ... RNEDNSL … LCDNVEV .. YADDGGV ...

$\begin{array}{cccc}\vdots & \vdots & \vdots & \vdots\end{array}$

$\mathbf{F}$

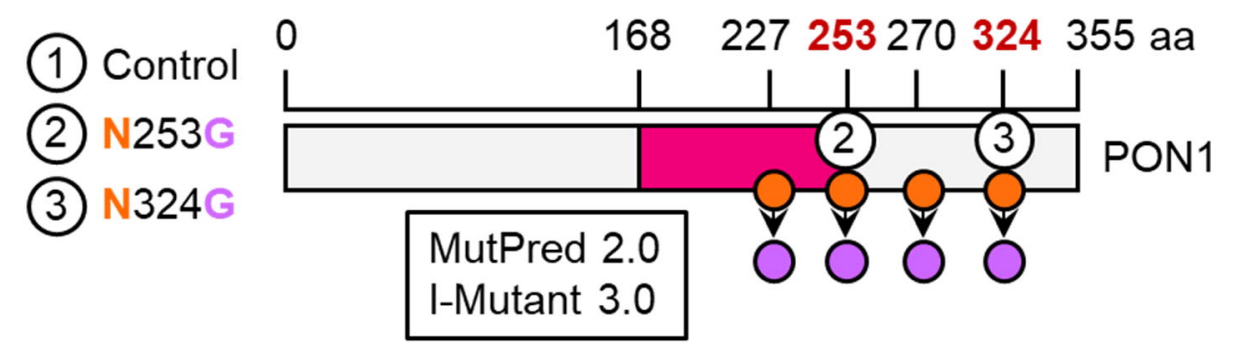
$\Delta \Delta \mathrm{G}$ value prediction: $-1.27 \mathrm{Kcal} / \mathrm{mol}$ Loss of $\mathrm{N}$-linked glycosylation $(P=\mathrm{NS})$

Change in protein stability: decrease $\Delta \Delta G$ value prediction: $-0.56 \mathrm{Kcal} / \mathrm{mol}$ Loss of N-linked glycosylation $(P=9.3 e-05)$ Ф Altered transmembrane protein $(P=1.6 \mathrm{e}-03) \mid$

Change in protein stability: decrease $\Delta \Delta \mathrm{G}$ value prediction: $\mathbf{- 1 . 2 4 \mathrm { Kcal } / \mathrm { mol }}$

Loss of $\mathrm{N}$-linked glycosylation ( $P=\mathrm{NS}$ ) Altered transmembrane protein $(P=N S)$

Change in protein stability: decrease $\Delta \Delta \mathrm{G}$ value prediction: $-0.76 \mathrm{Kcal} / \mathrm{mol}$ Altered transmembrane protein $(P=1.5 e-05)$ Altered ordered interface Gain of loop
Change in protein stability: decrease Loss of N-linked glycosylation $(P=7.4 \mathrm{e}-03)$ 岕

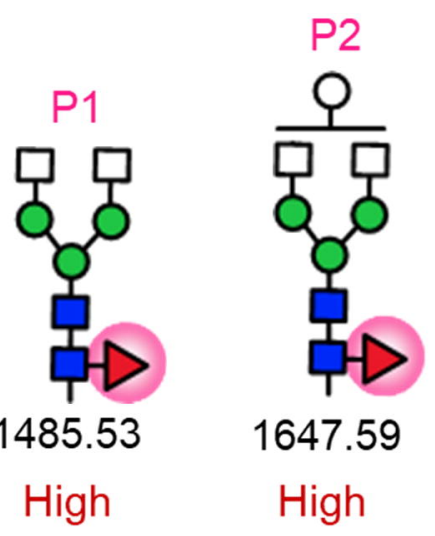

G

AAL blotting after IB: PON1

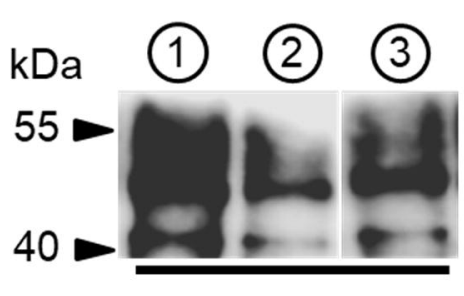

IP: anti-PON1

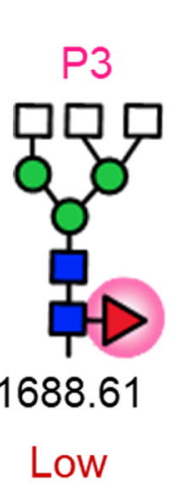

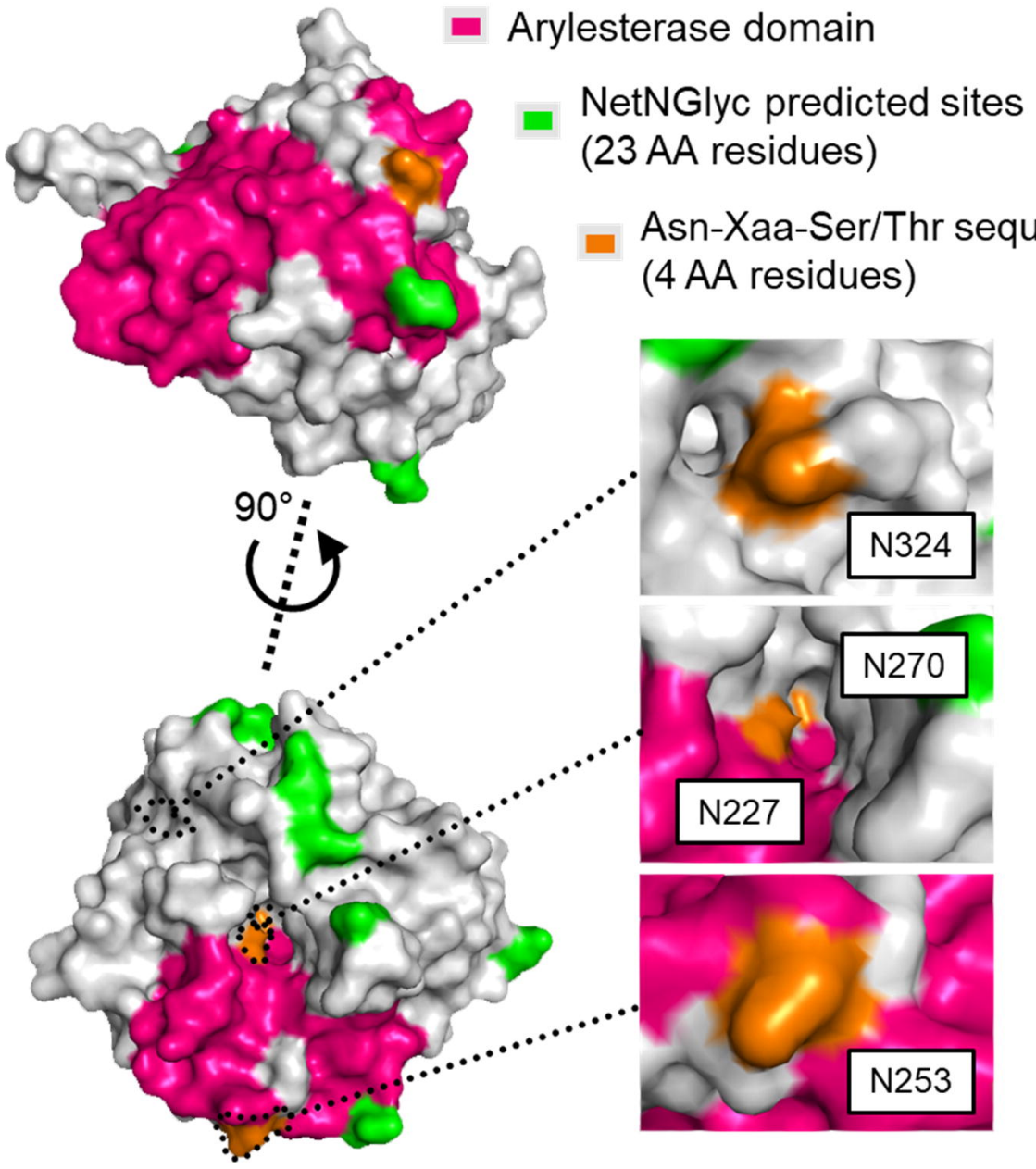
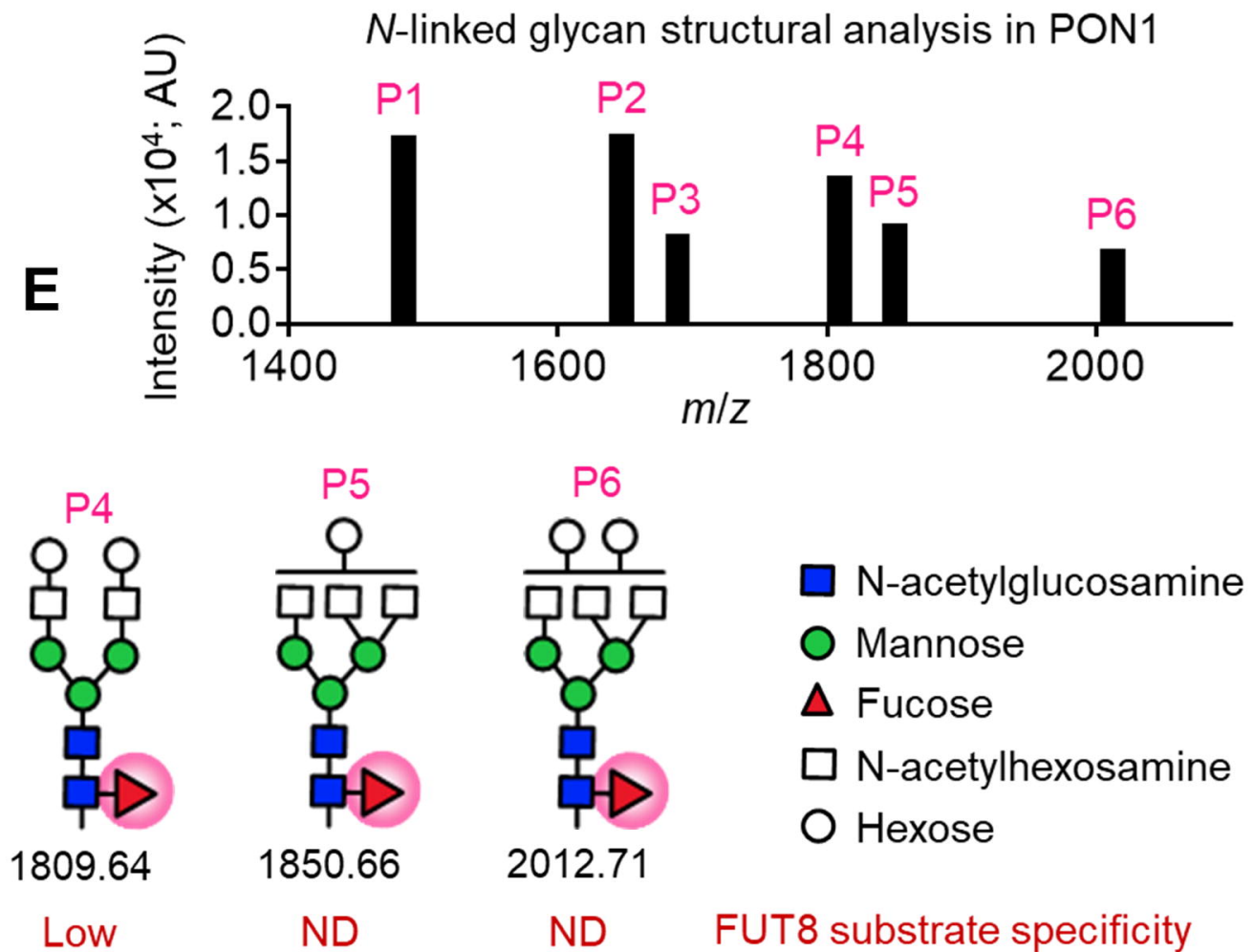

$\mathrm{N}$-acetylglucosamine

O Mannose

$\triangle$ Fucose

$\square$ N-acetylhexosamine

O Hexose

ND

FUT8 substrate specificity

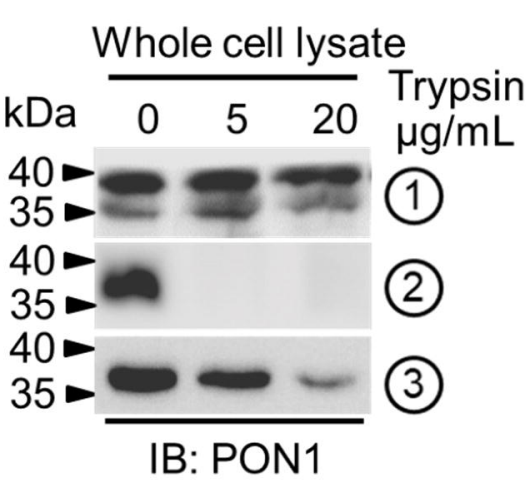

J $\underset{\text { fractionation }}{\text { Golgi/ER }} \rightarrow$ Trypsin $\rightarrow \begin{aligned} & \text { PON1 } \\ & \text { ELISA }\end{aligned}$

J

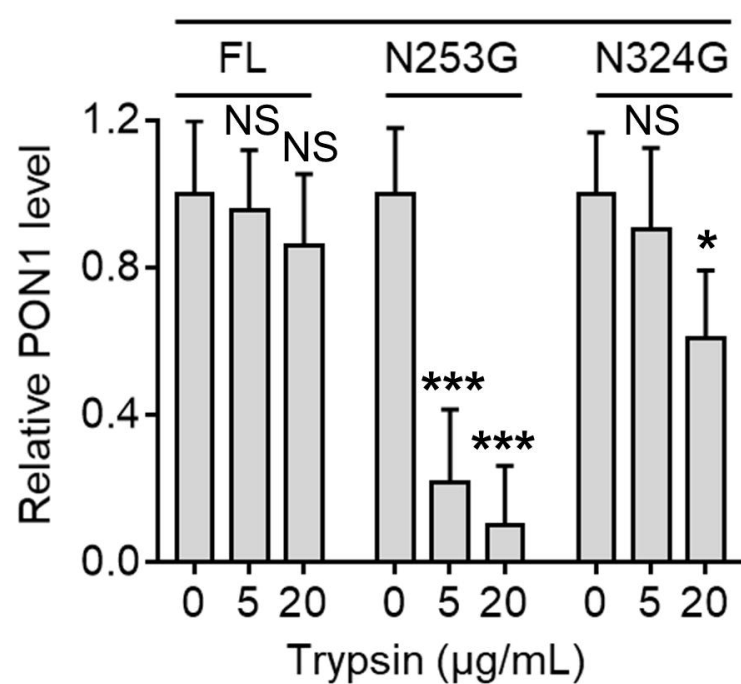

K Nascent polypeptide synthesis

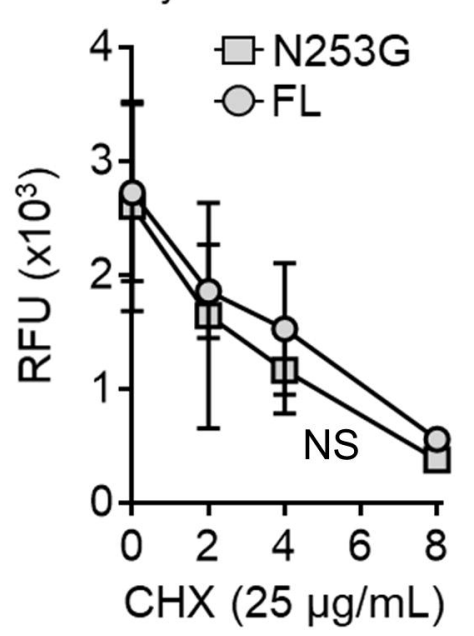

L

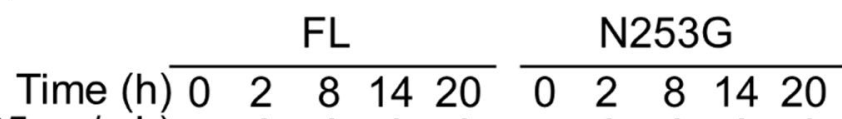

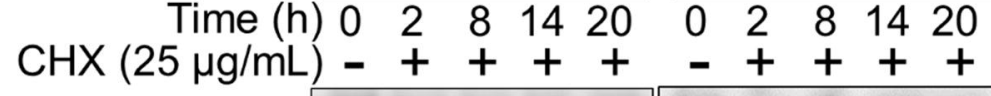

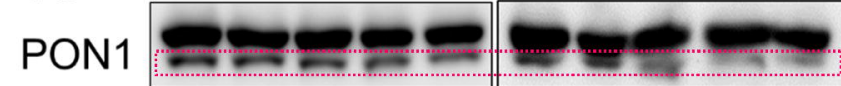

GAPDH

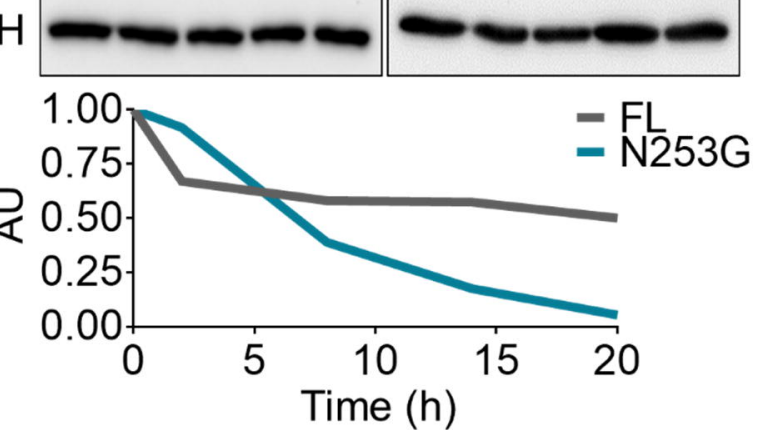

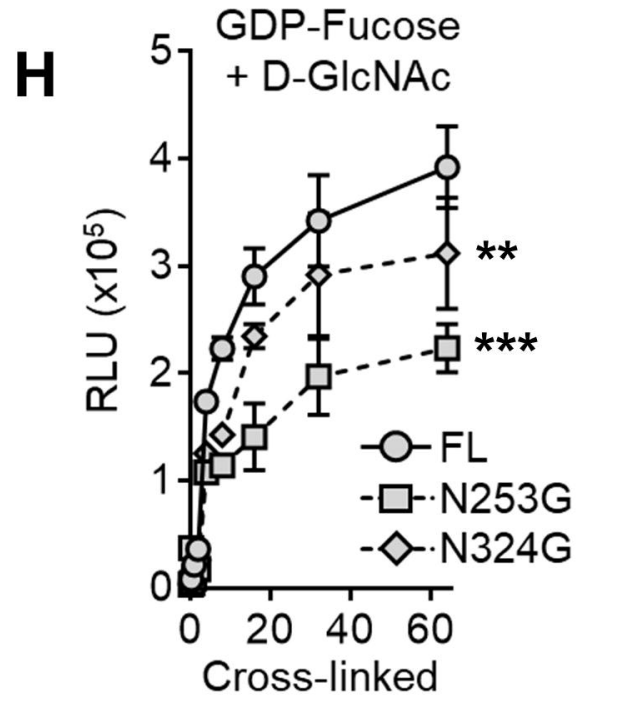

Cross-linked FUT8-PON1 lysates ( $\mu \mathrm{g} /$ reaction)

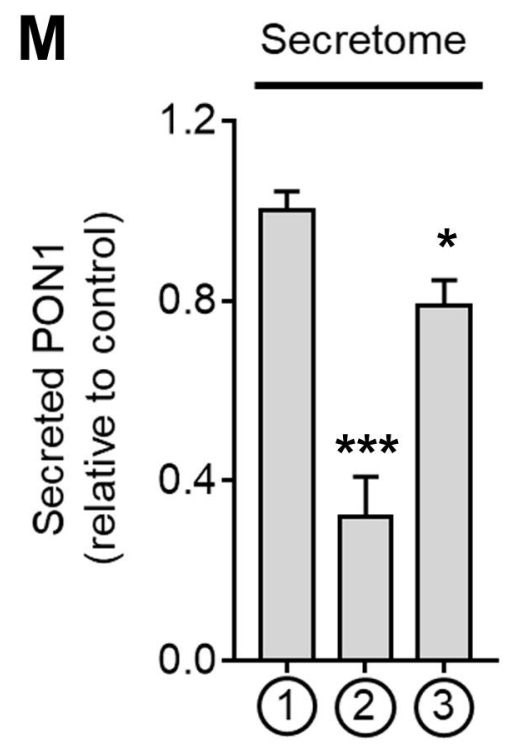




\section{Figure 5.}

A

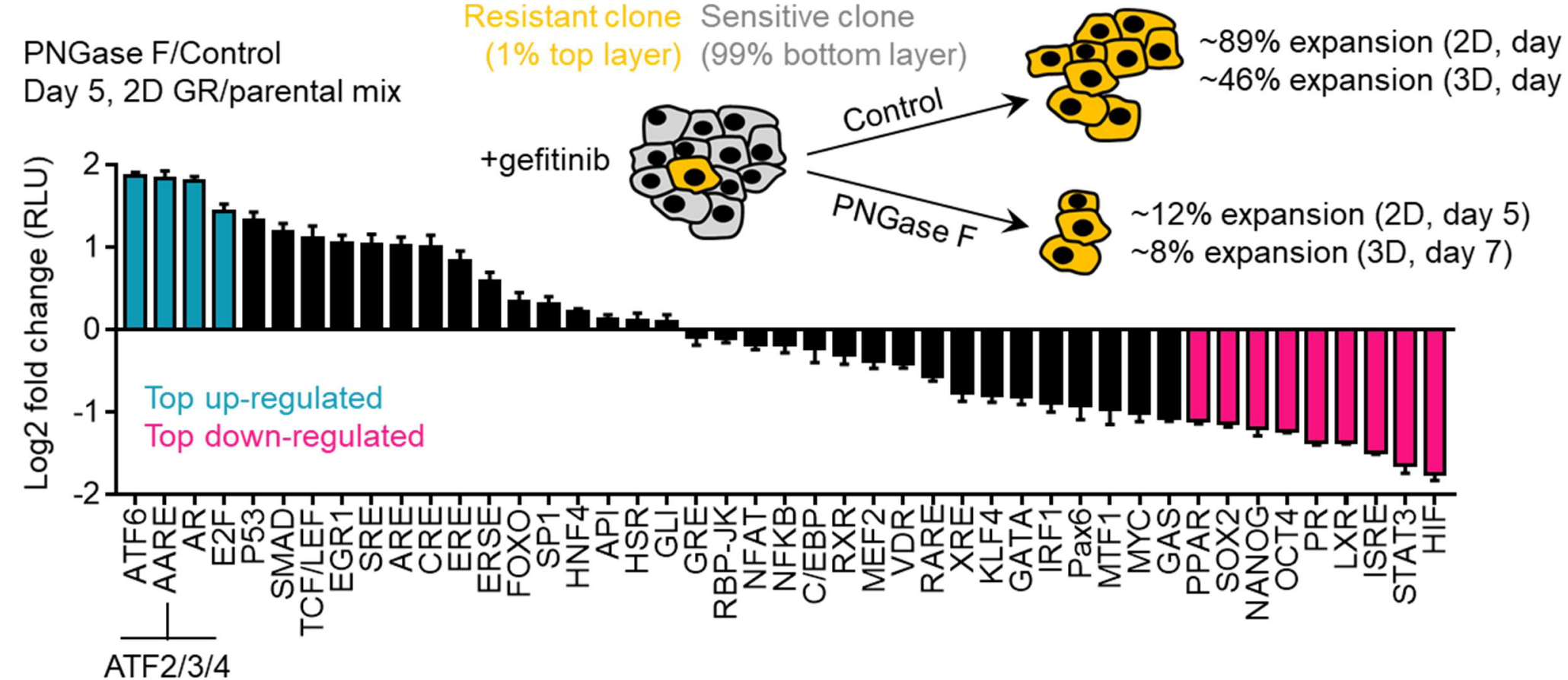

3D GR/parental mix

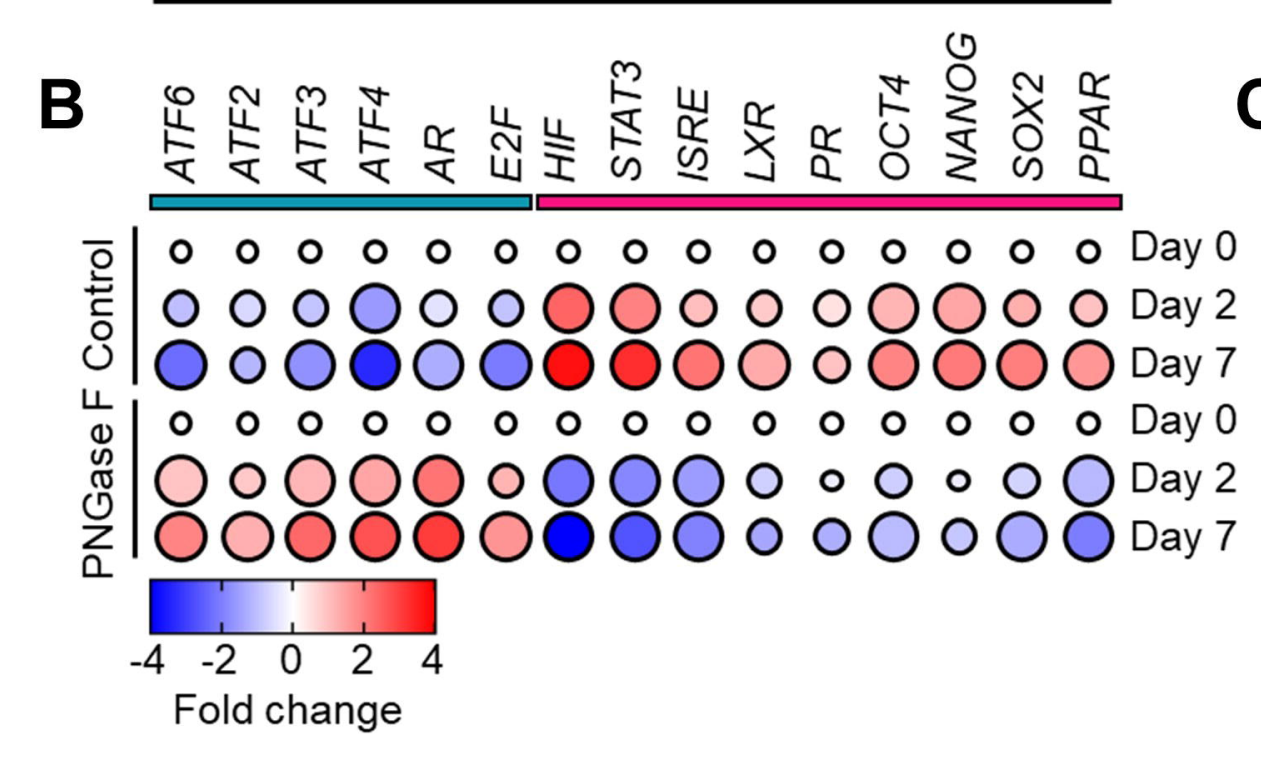

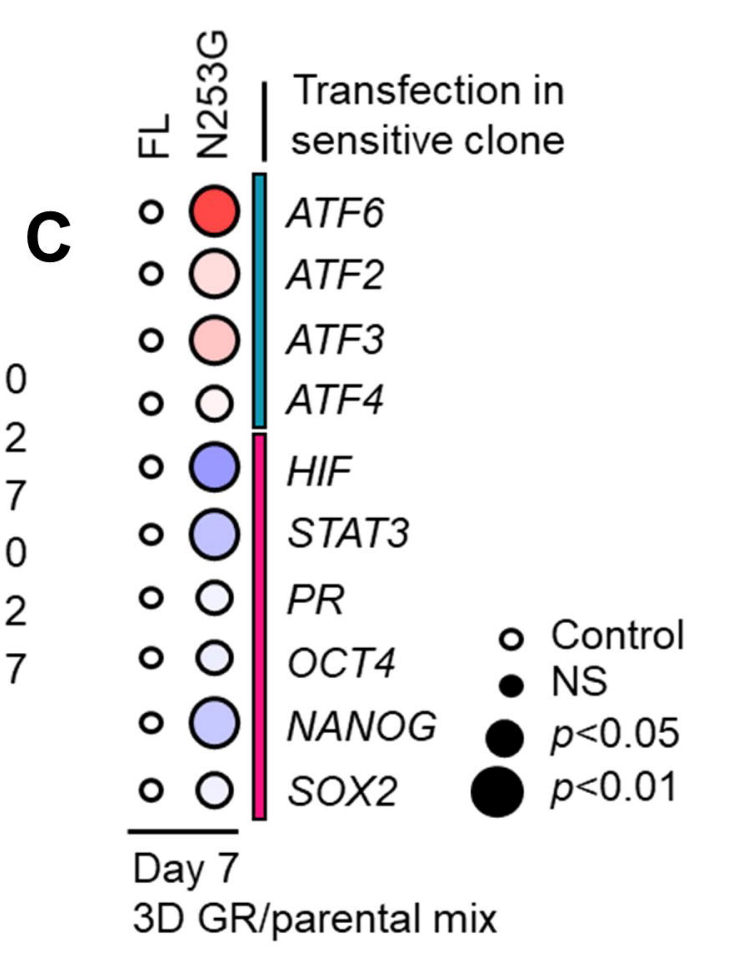

D

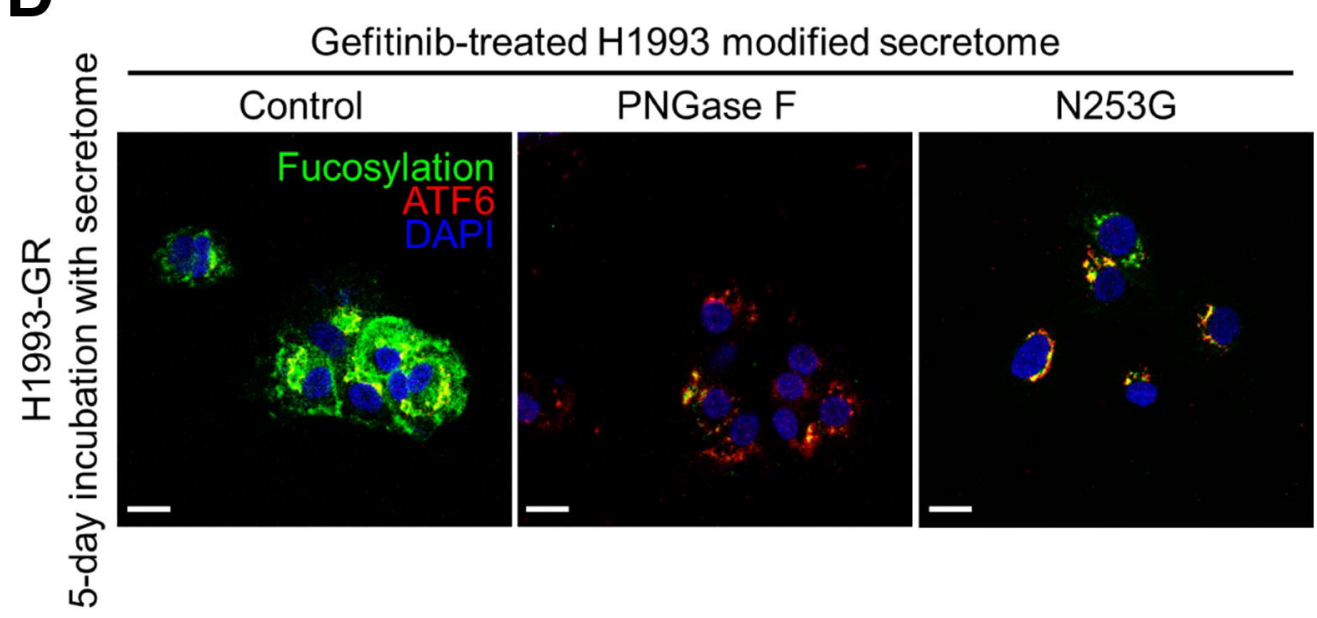

F

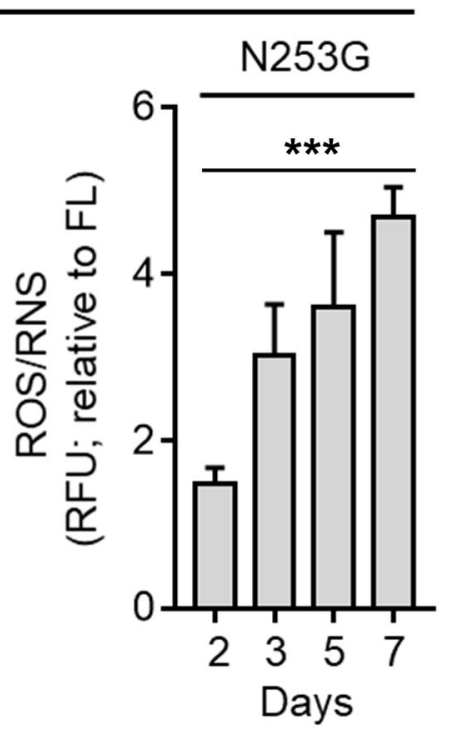

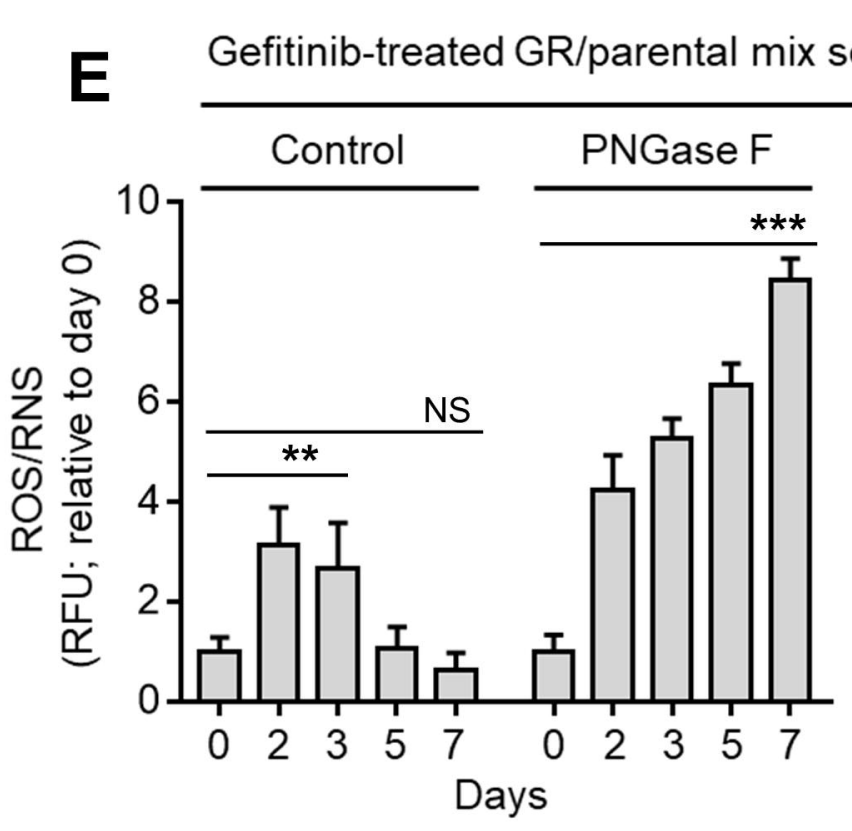

$\underset{2}{2 H}$

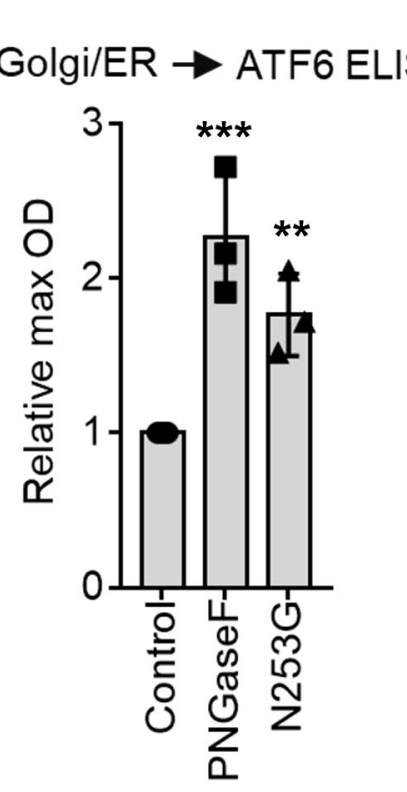

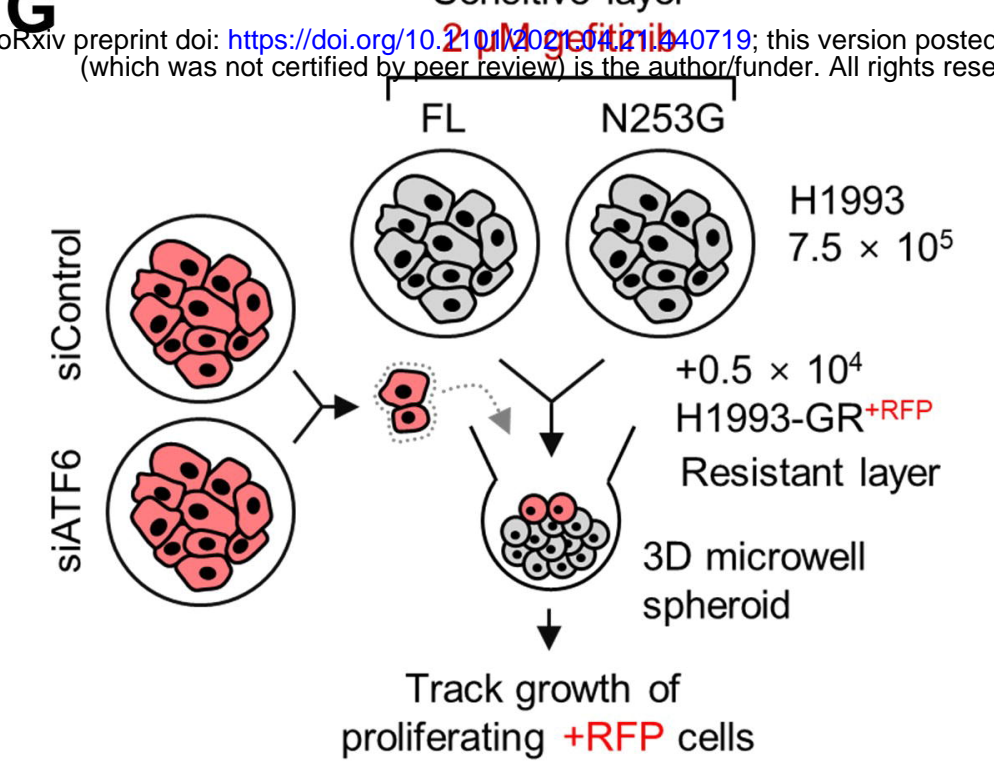

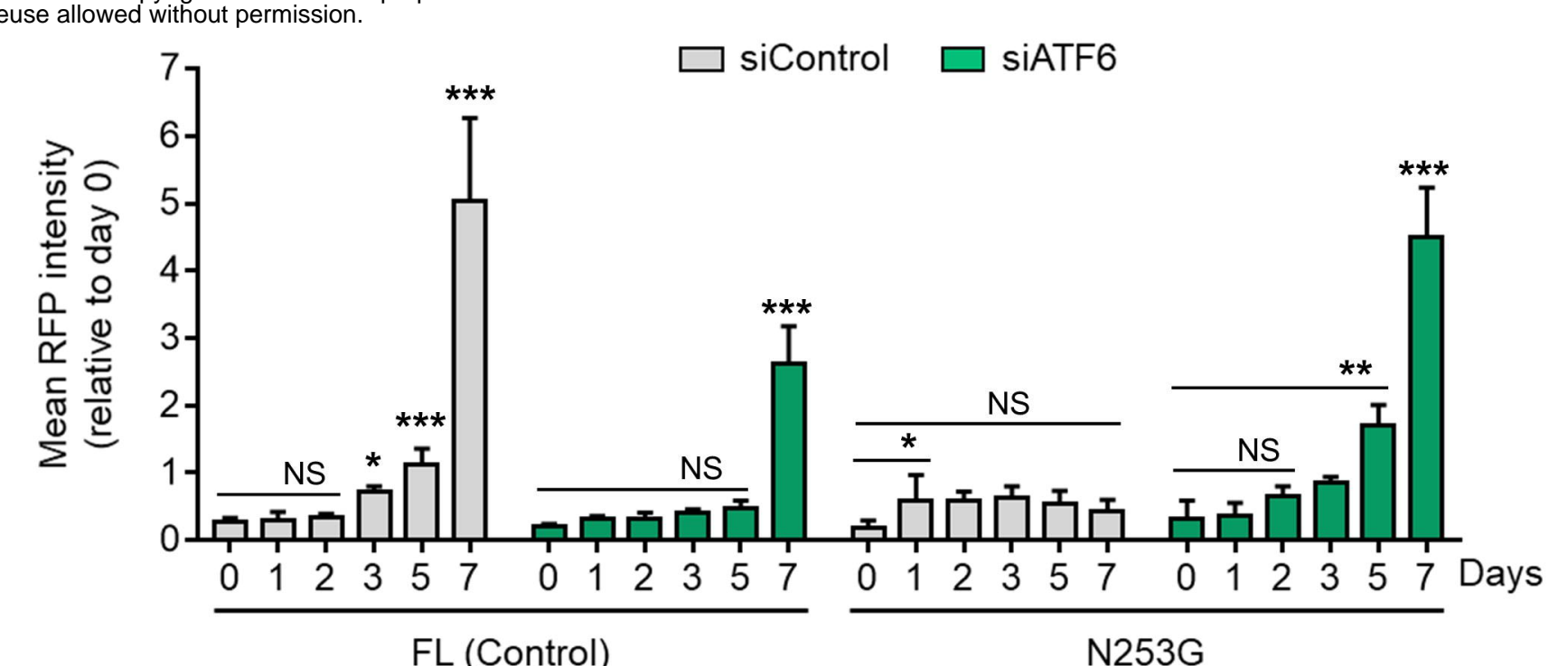

J

$\overline{\text { Modified secretome }}$ Secretome PON1

$\frac{\text { source }}{\text { H460-PON1 Up }}$

H1299-PON1 Up

A549-shPON1 Not significant

H460-PON1-shSLC35C1 Down

H460-PON1(+PNGase F) Down

H460-PON1(+N253G) Down

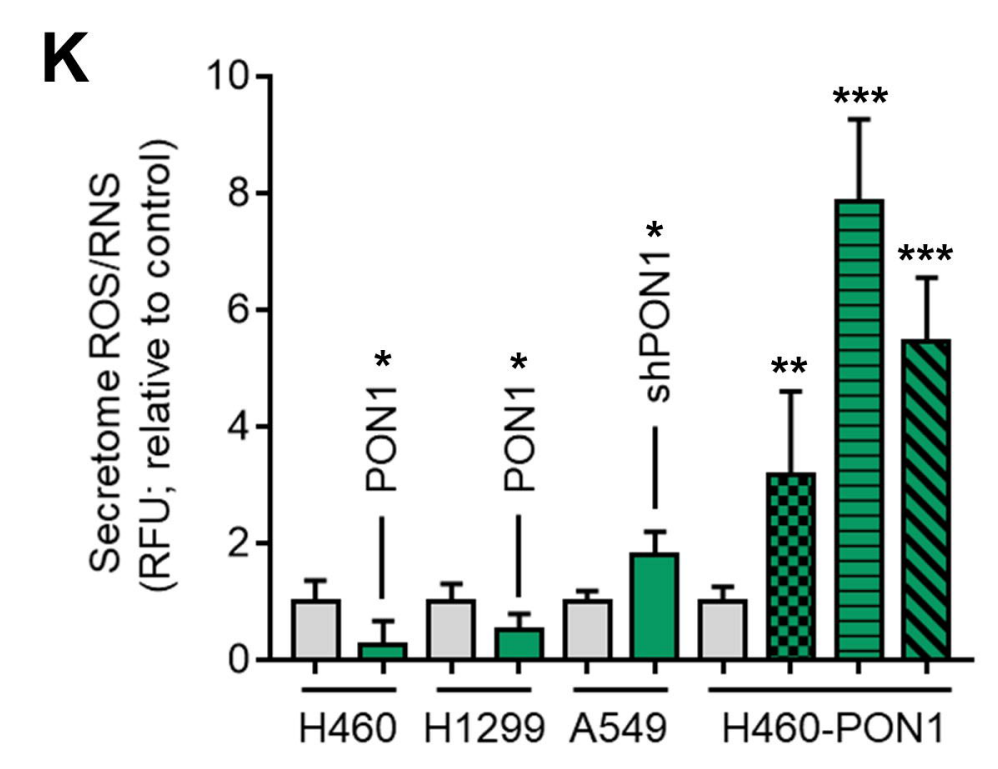

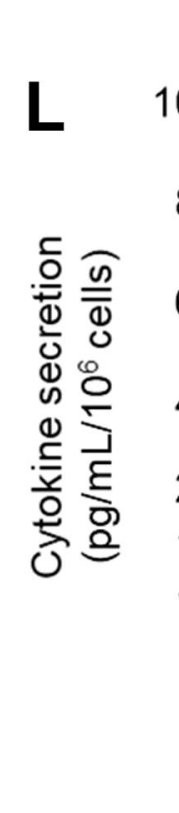

I

Day 5, 2D GR/parental mix


$\square$ Control/shControl

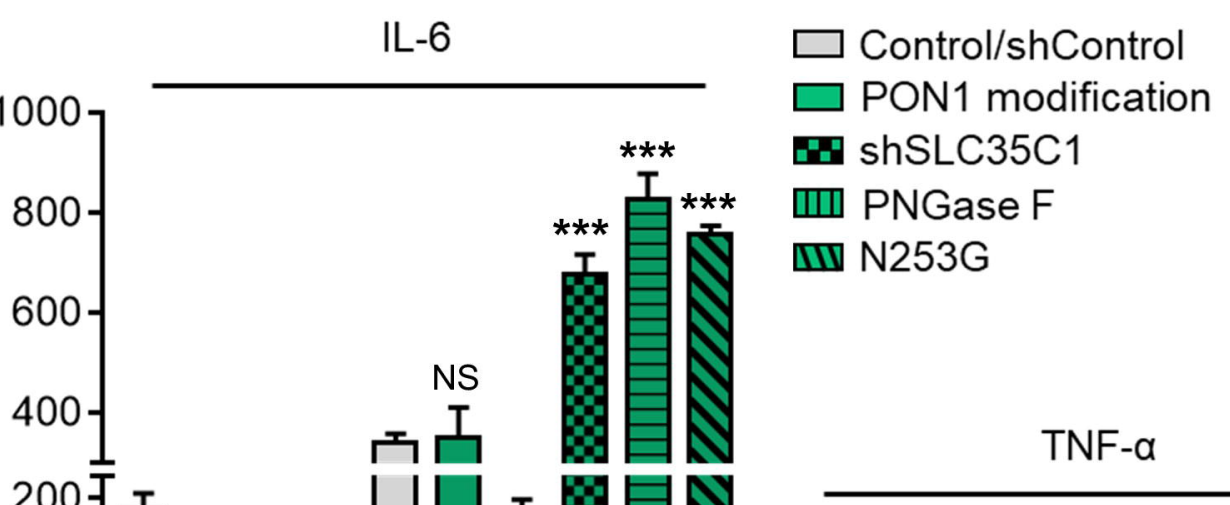

GM-CSF

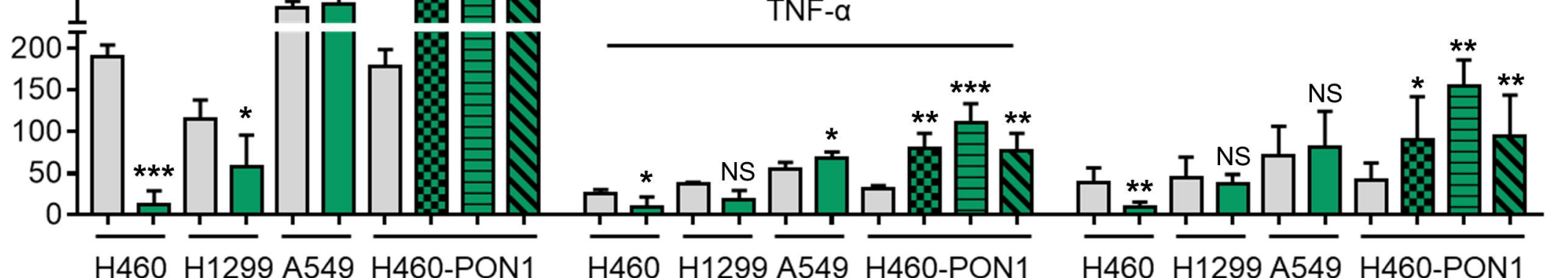

$\overline{\mathrm{H} 460} \overline{\mathrm{H} 1299} \overline{\mathrm{A} 549} \overline{\mathrm{H} 460-\mathrm{PON} 1} \overline{\mathrm{H} 460} \overline{\mathrm{H} 1299} \overline{\mathrm{A} 549} \overline{\mathrm{H} 460-\mathrm{PON} 1} \overline{\mathrm{H} 460} \overline{\mathrm{H} 1299} \overline{\mathrm{A} 549} \overline{\mathrm{H} 460-\mathrm{PON} 1}$

$\mathbf{N}$

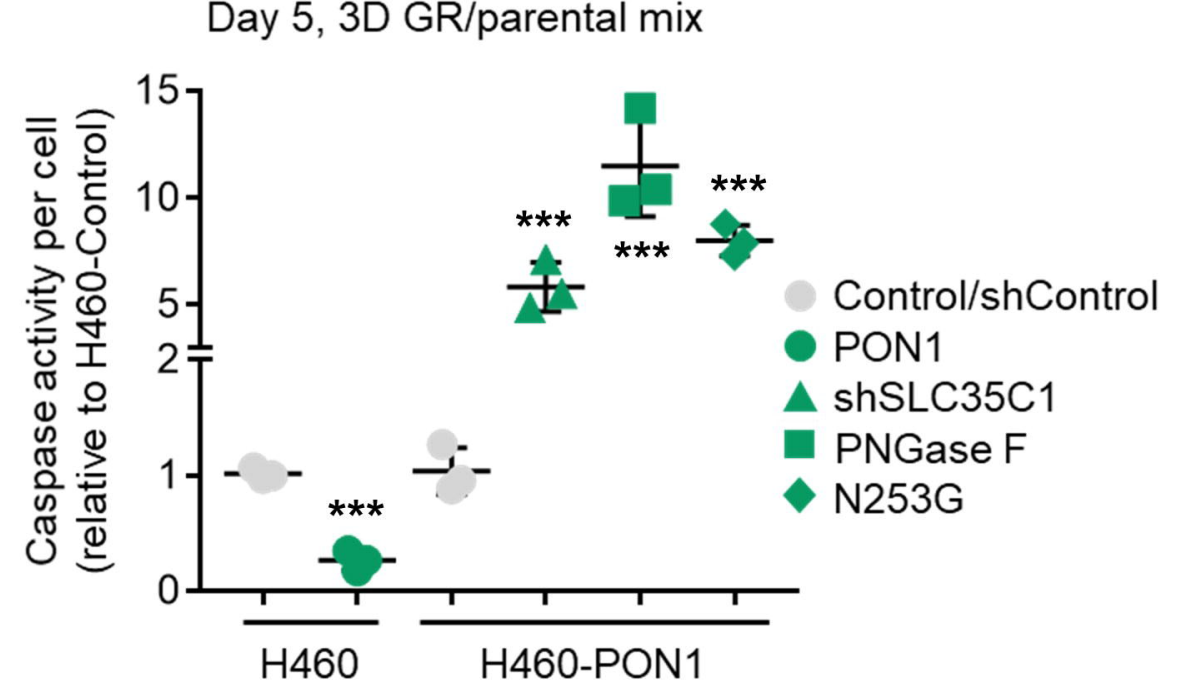


Figure 6.

A Enriched fucosylation \& Deglycosylation/ o

PON1 fucosylation inhibition $\uparrow$ (prior to conditioning) $48 \mathrm{~h}$ culture

- Gefitinib sensitive $\mathrm{H} 1993$ or H460(-CC/PON1) cells

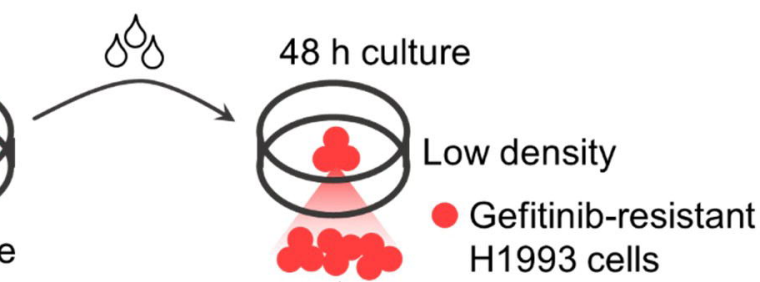

$\stackrel{\downarrow}{\rightleftharpoons}$ Resistant $\widetilde{\rightleftharpoons}$ cell mRNA library RNA-seq

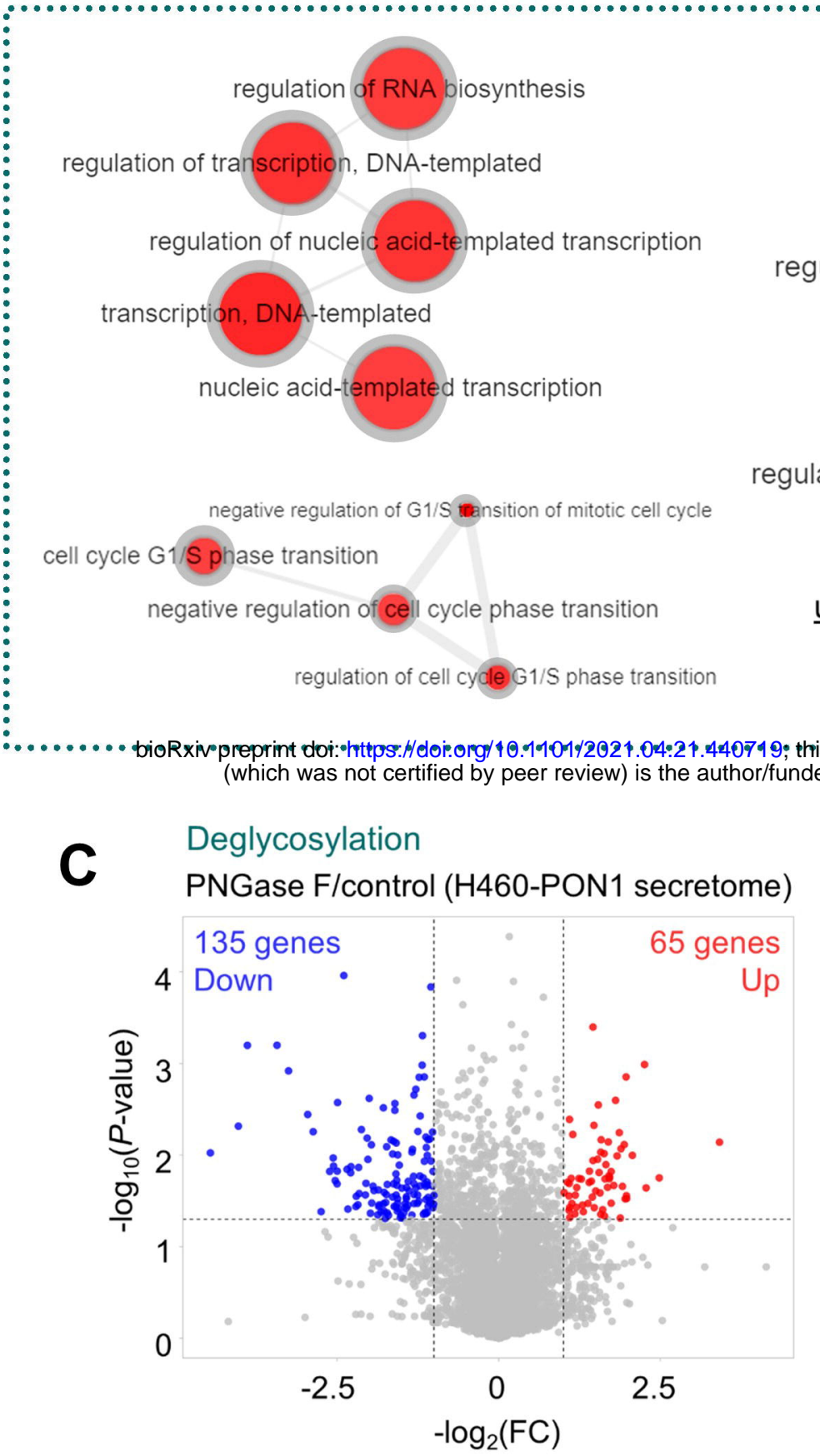

E

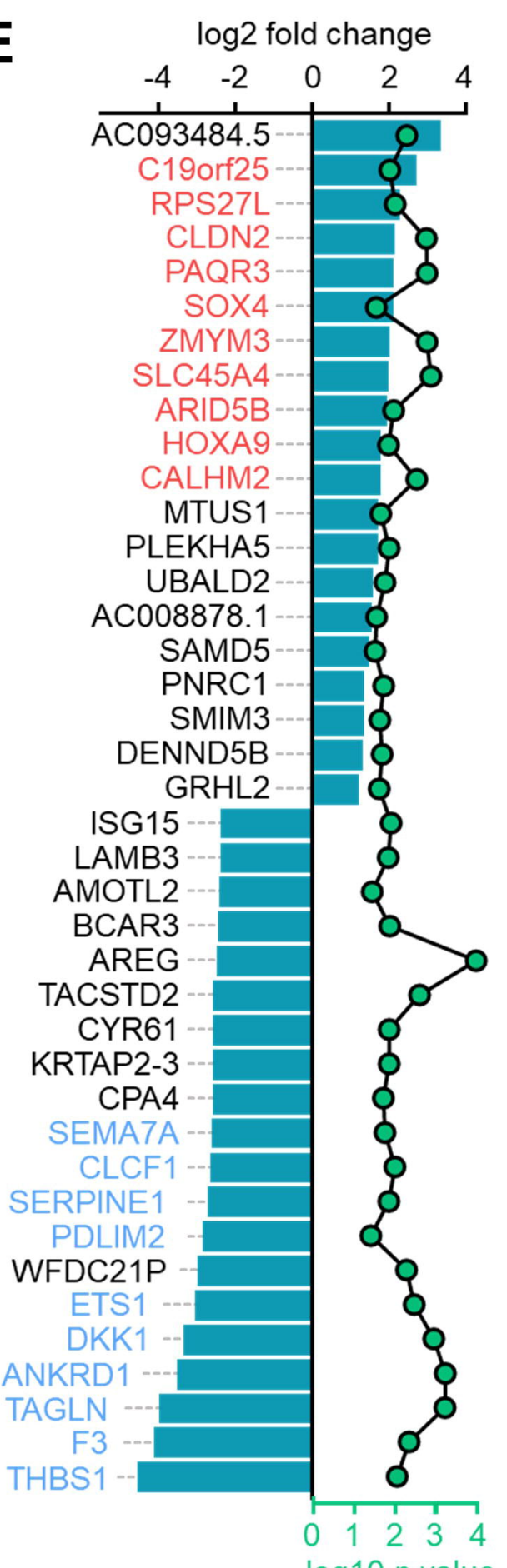

B

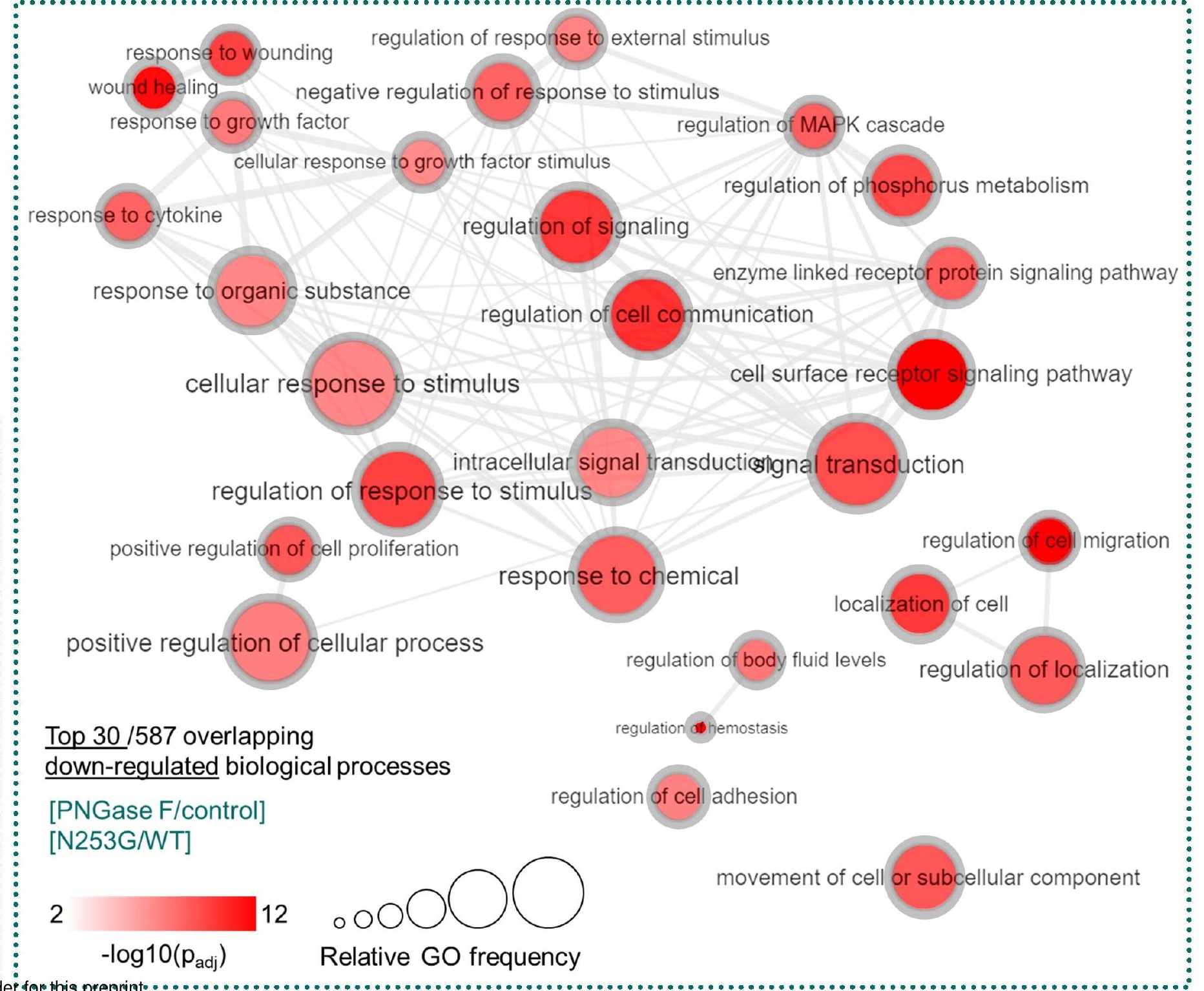

Top 14/30 overlapping up-regulated biological processes [PNGase F/control] [N253G/NT]

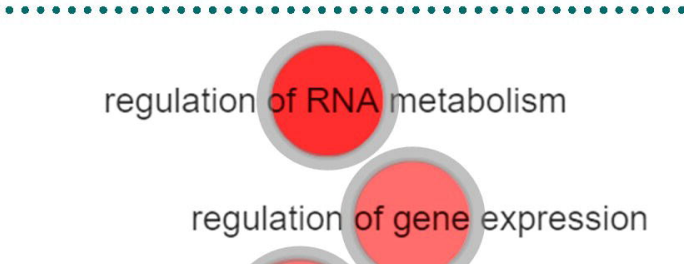

regulation of primary metabolism

regulation of cellular biosynthesis

gulation of macromolecule biosynthesis

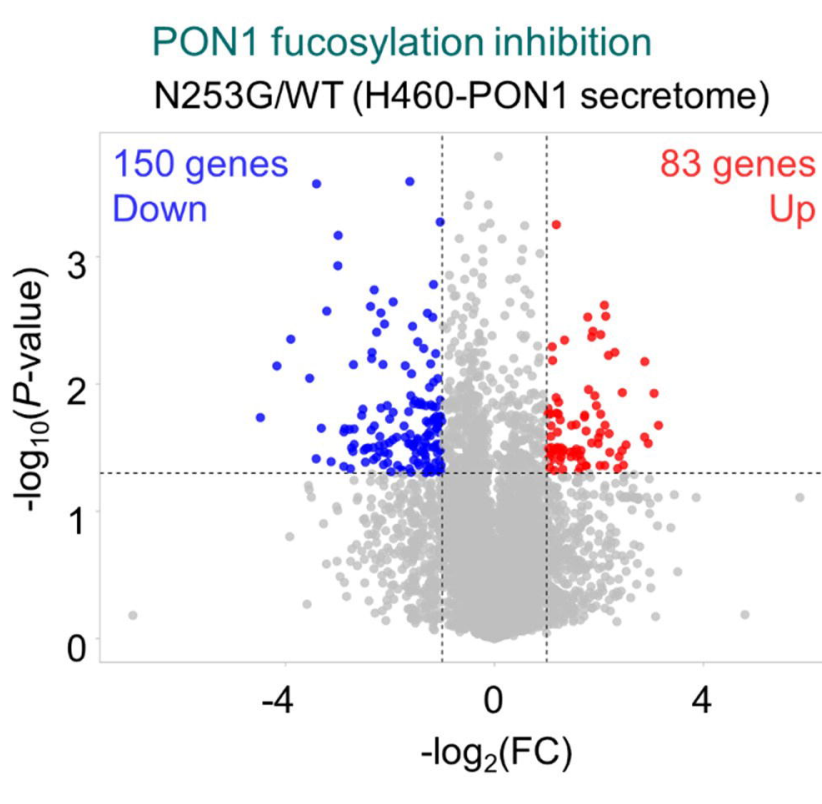

$\mathbf{F}$

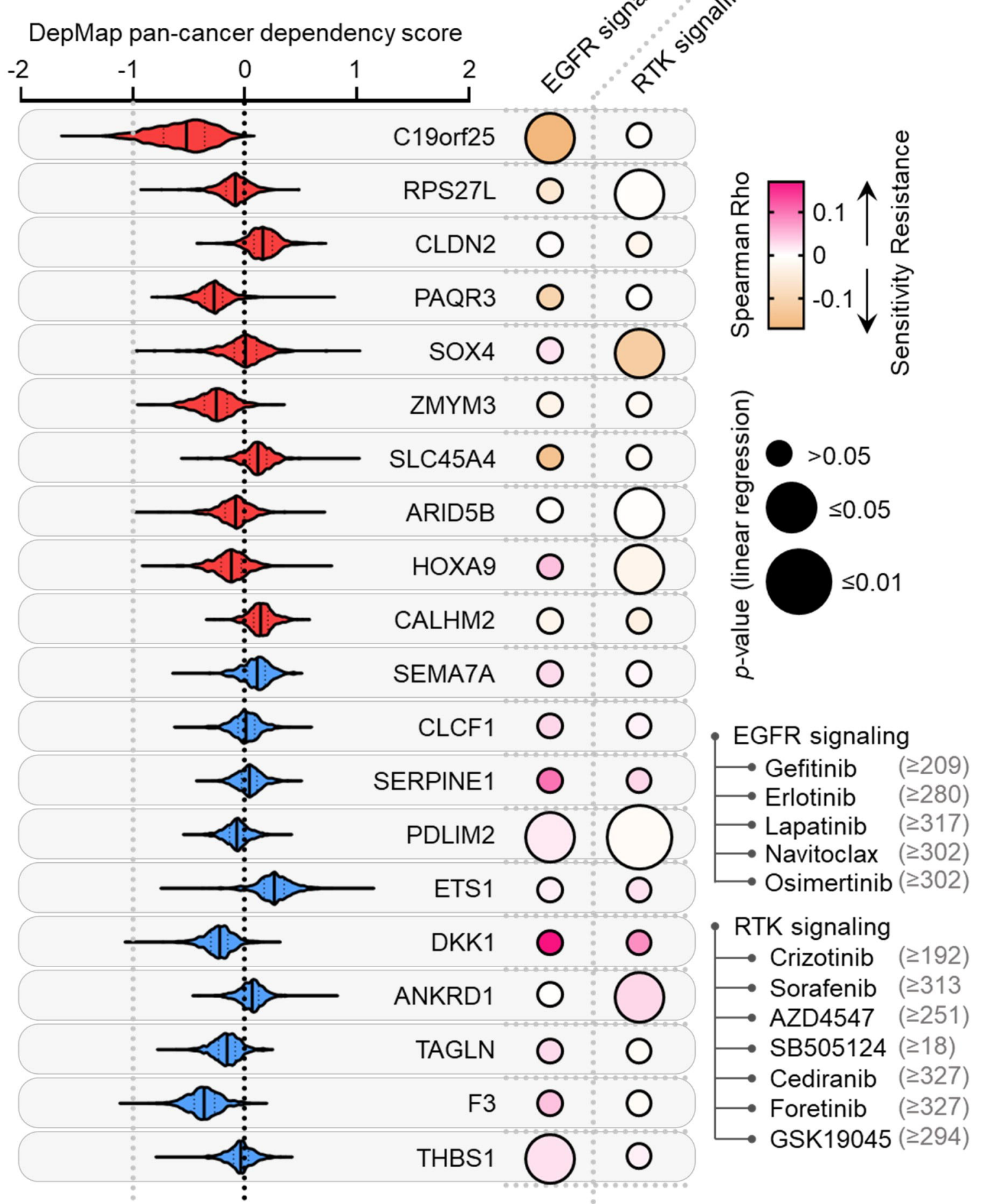

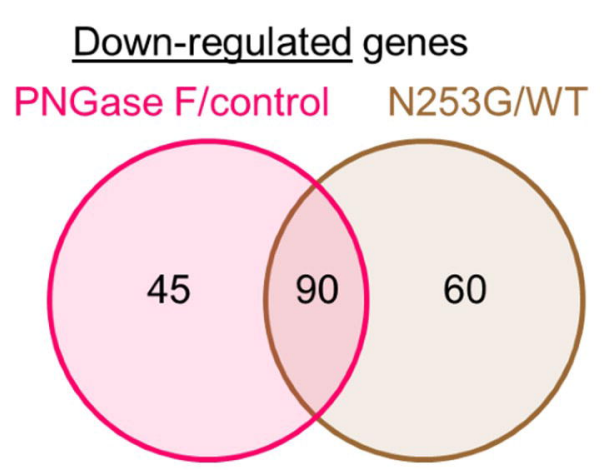
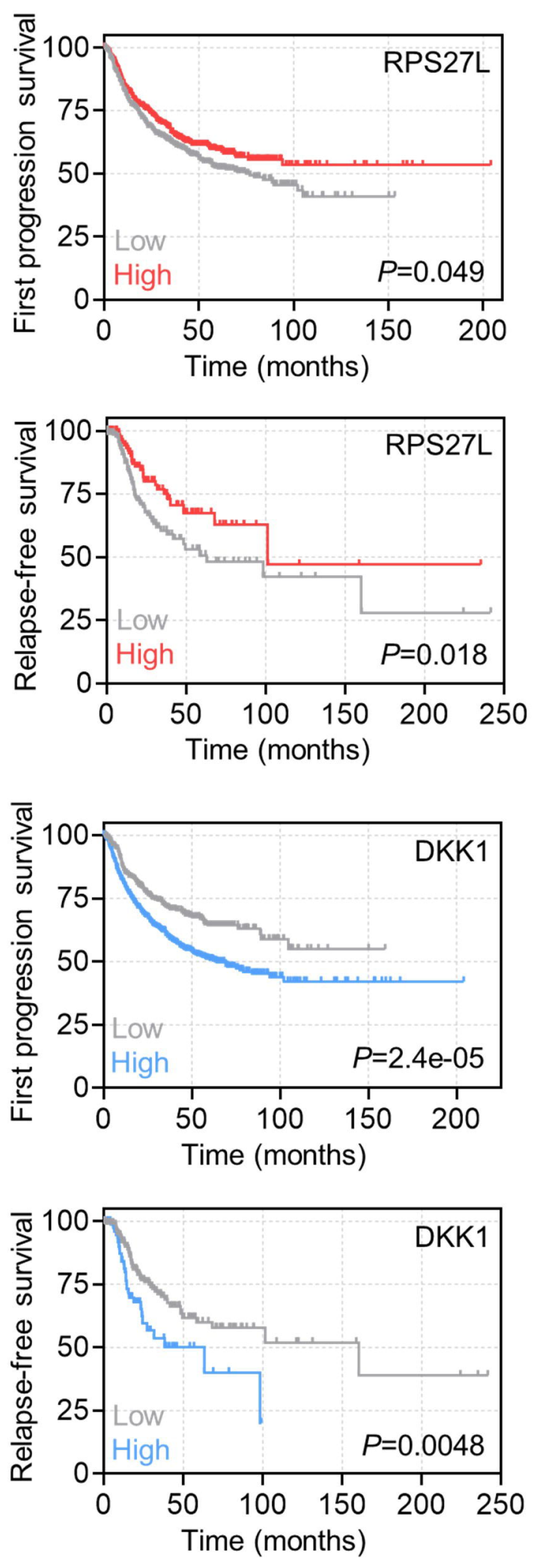